\title{
THE RESTRICTED PROBLEM OF THREE BODIES.
}

\author{
By George D. Birkhoff (Cambridge, Mass.).
}

Adunanza del 23 agosto 1914.

Thorough investigation of non-integrable dynamical problems is essential for the further progress of dynamics. Up to the present time only the periodic movements and certain closely allied movements have been treated with any degree of success in such problems, but the final goal of dynamics embraces the characterization of all types of movement, and of their interrelation.

The so-called restricted problem of three bodies, in which a particle of zero mass moves subject to the attraction of two other bodies of positive mass rotating in circles about their centre of gravity, affords a typical and important example of a non-integrable dynamical system. It is this problem which I consider in the present paper.

Part I (SS I-5) deals with the differential equations of the problem.

As ordinarily given, the equations of motion $\left(\oint_{I}\right)$ admit of three singular points corresponding to the collision of the particle with either body and to its rejection to infinity. It was the notable achievement of Levi-Civita ${ }^{x}$ ) to have discovered that the

I) T. Levi-Civita, Sur la résolution qualitative du probleme restreint des trois corps [Acta Mathematica, t. XXX (1906), pp. 305-327]. See also an earlier paper by the same author, Traiettorie singolari ed urti nel problema ristretto dei tre corpi [Annali di Matematica, ser. III, vol. IX (I904), pp. I-32].

[Note added January $3 \mathrm{I}$, I9I4. As I have been kindly apprised by Professor Levi-Civita, he has found more recently that a regularization at collision with both bodies had been given by $T$. N. THIELE, Recherches numeriques concernant des solutions périodiques d'un cas special du probleme des trois corps (Troisième mémoire) [Astronomische Nachrichten, vol. CXXXVIII (1895), pp. I-Io], who, however, deduced no theoretical consequences therefrom. This regularization, less simple then that of Levi-Civita and also falling under the type treated by me in $\ 2$, is entirely distinct from the algebraic regularization given in $₫ 5$, and used later in the paper.

Dr. G. M. GReEN and I have both noted that all of these forms of the equations of motion become canonical, for a given value $C$ of the constant of JACOBI, if the variables of the first set are $u, v(\$ 2)$, and of the conjugate set are $\bar{u}, \bar{v}$, given by

$$
\bar{u}=\left(\frac{d x}{d t}-y\right) x_{u}+\left(\frac{d y}{d t}+x\right) y_{u}, \quad \bar{v}=\left(\frac{d x}{d t}-y\right) x_{v}+\left(\frac{d y}{d t}+x\right) y_{i:},
$$


equations of motion admit of regularization so far as collision with a given one of the bodies is concerned. By the aid of this regularization it became possible to determine the character of the motion near collision without difficulty.

On the basis of a new transformation of variables $(\$ 2)$ applicable to a considerable range of dynamical problems, I obtain the well-known equations of normal and tangential displacement $(\$ 3)$, the equations of Levi-Civita although not in canonical form $(\$ 4)$, and a new form of the equations of motion in which the only singularity corresponds to the rejection of the particle to infinity $(\$ \varsigma)$.

In Part II ( $\$$ S6-8) the nature of the manifolds of states of motion is considered; by a state of motion I mean the position of the particle together with its vector velocity at an instant.

The new form of the equations affords a non-singular representation of the manifolds of states of motion for any value of the constant of JACOBI, and allows one to represent the movements under consideration as the stream lines of a three-dimensional flow in non-singular steady motion $(\$ 6)$.

In this way a characterization of the states of motion in the six possible cases may be made without difficulty $(\$ \subseteq 7,8)$. For example, it is found that when the particle is confined to an oval of zero velocity about one of the bodies, the manifold is in one-to-one continuous correspondence with the points inside of and on a sphere, under the proviso that diametrically opposite points are counted as identical; under the same proviso, it turns out that, when the oval of zero velocity includes both bodies, the manifold of states of motion is in one-to-one continuous correspondence with the points between and on two concentric spheres.

The case of the problem in which one of the two bodies is of mass zero admits of explicit solution. Porncare ${ }^{2}$ ) generalized from this limiting case and was led to a representation in the space of inversion as long as the particle was confined to an oval of zero velocity containing one of the bodies. It was not seen by him however that this representation is two-to-one, "a fact in accord with the one-to-one representation by a sphere stated above ${ }^{3}$ ).

The further results of the present paper are concerned only with the case in which the particle is confined to such an oval.

The same generalization led Poncaré to the fundamental concept that the restricted problem of three bodies admits of reduction to the transformation of a ring

while the principal function is

$$
\left(\frac{\mathrm{I}}{2}\left[\left(\frac{d x}{d t}\right)^{2}+\left(\frac{d y}{d t}\right)^{2}\right]-\left[\Omega(x, y)-\frac{\mathrm{I}}{2} C\right]\right)\left|f^{\prime}(w)\right|^{2},
$$

and the independent variable is $\tau]$.

2) H. Porncaré, Les méthodes nouvelles de la Mécanique Céleste, vol. III (Paris, Gauthier-Villars, I899), pp. I96-200, 372-381.

3) H. Poincaré, Sur un théorème de Géométrie [Rendi zonti del Circolo Matematico di Palermo, t. XXXIII ( ${ }^{\circ}$ semestre Ig!2), pp. 375-407]. 
into itself. Part III ( $\iint 9$-I 5 ) of the present paper is devoted to the further investigation of this concept.

I commence with a consideration of the case in which one of the bodies is of mass zero, and arrive at a geometrical representation of the ring in this case $(\mathbb{S} 9,10)$.

In order to generalize this interpretation certain properties of the family of direct and retrograde periodic orbits are established ( $\$ S$ II, I2). As the constant of JACOBI increases from the given value to infinity a continuous ring of such periodic orbits is obtained. The states of motion belonging to the ring for the given value of this constant are the states of positive tangency to these auxiliary orbits. If a particle be projected from any point $K$ in the plane of the motion tangent to the auxiliary orbit through the point, the particle will become tangent again to other such orbits at points $L$, $M, \ldots$ infinitely often. The transformation of the ring is defined as that which takes $K$ into the first such point $L(\$ \mathrm{r} 3)$.

In this manner a complete representation of the ring transformation in the plane of the motion becones possible. The ring employed may of course also be thought of as situated in the space representing the manifold of states of motion. It differs from the ring obtained by POINCARE merely in its simple and more specific character.

A fundamental property of this and similar transformations, noted by PoIncaré, is that an area integral is invariant. A further property, which I have found to hold in the restricted problem of three bodies, is that the transformation is the product of two involutory transformations ( $($ I 4 ). The latter property is the important one for the present paper.

The construction of a ring outlined above is valid only for suitably restricted values of the masses. A necessary and sufficient condition for the existence of a ring can be found on the hypothesis that direct and retrograde orbits of simple type, corresponding to the two boundaries of the ring, exist ( $(15)$.

Part IV ( $\$ \int$ I6-2I) is given over to the consideration of the periodic orbits. Later I expect to publish the results which I have obtained concerning the general orbit.

The question of the existence of periodic orbits is of great importance. The notable work of HiLl ${ }^{4}$ ) and DARwIN ${ }^{5}$ ) leaves no practical doubt that direct periodic orbits of simple type continue to exist through wide ranges of valucs of the masses and of the constant of JACOBI, Moreover, Moulton ${ }^{\circ}$ ) has observed that retrograde orbits cannot vary through a cusped form under certain conditions, and so seem to have a peculiar quality of permanence. His interesting conjectures concerning these orbits have been borne out by later computations made under his direction.

4) G. W. HiLl, Researches in the Lunar Theory [American Journal of Mathematics, vol. I (1878), pp. 5-26, 129-147, 245-260].

5) G. H. Darwin, Periodic Orbits [Acta Mathematica, vol. XXI (1897), pp. 99-242].

o) F. R. Moulton, Relations among Families of Periodic Orbits in the Restricted Problem of Three Bodies [Proceedings of the fifth International Congress of Mathematicians, Cambridge, I913, vol. II, pp. I82-187]. 
Notwithstanding these facts, however, the existence of these direct and retrograde orbits has only been rigorously established for sufficiently small values of one of the masses, or for a sufficiently large value of the constant of JACOBI ${ }^{7}$ ).

By a simple method I have been able to demonstrate that, as long as the particle is confined to an oval of zero velocity about one of the bodies, there necessarily exists at least one retrograde orbit of simple type ( $\left.\iint_{1} 6,17,18\right)$. .

The direct orbits do not appear to he amenable to a like attack. I have, however, been able to establish that, for sufficiently small values of one of the masses, the ring transformation of PolNCARÉ may be replaced by the transformation of a discoid into itself whose only boundary corresponds to the retrograde orbit, and that, as long as this construction is possible, there will necessarily be one direct orbit of simple type $(\$$ I9).

The balance of evidence seems to render it probable that this new form of transformation continues to exist as long as there is an oval of zero velocity about each of the two masses. The ring transformation, on the contrary, necessarily breaks down when the direct orbit becomes unstable.

In a last paper Porncaré ${ }^{3}$ ) established, that, if a certain geometric theorem were true, the existence of a ring transformation carried with it the existence of an infinite number of periodic solutions of the restricted problem of three bodies. This geometric theorem was later proved by $\mathrm{me}^{\mathbf{8}}$ ). The property of the ring used is that an area integral remains invariant under the transformation. Since the earlier work of PorNCARÉ had only established the existence of an arbitrarily large number of periodic solutions for sufficiently small values of one of the masses, this conclusion constituted a definite step in advance, made possible only by the reduction of the restricted problem to the transformation of a ring into itself.

It was stated above that this transformation is the product of two involutory transformations. The existence of an infinite number of symmetric periodic orbits flows intuitively from this fact; by its means also very definite conclusions concerning the characteristic properties of these orbits, and concerning their distribution in the plane of the motion, may be reached $(\$ 20)$.

The property used by Poincaré enters, however, into the proof of certain geometric facts $(\ 21)$. For example, as long as the ring exists, it may be proved that, for the given value of the constant of JACOBI and a given point in the plane, an initial direction of projection may be chosen in an infinite variety of ways so that the particle passes through the initial point at a later time.

7) A criterion for the existence of periodic orbits has been given by E. T. WhITTARER, On Periodic Orbits in the Restricted Problem of Three Bodies [Monthly Notices of the Royal Astronomical Society, vol. LXII (I90I-1902), pp. 346-352].

8) G. D. Birkhoff, Proof of Porncaré's Geometric Theorem [Transactions of the American Mathematical Society, vol. XIV (1913), pp. 14-22], Démonstration du dernier Théorème de Gécmélrie de Poincaré [Bulletin de la Société Mathématique de France, vol. XLII (1914), pp. I-I2] (translation). 


\section{PART I.}

THE DIFFERENTIAL EQUATIONS OF THE PROBLEM.

$$
\text { SI. }
$$

\section{The Usual Form of the Equations of Motion.}

The restricted problem of three bodies in its customary form deals with the relntive motion of a particle $P$ of zero mass lying in one and the same plane with two bodies $S$ and $J$, which attract each other and $P$ according to the Newtonian law, and rotate positively in circles about their center of gravity $O$. By a specialization of units the masses of $S$ and $J$ become $\mu$ and I $-\mu$ respectively, while the distance from $S$ to $J$, the angular velocity of $S$ and $J$, and the gravitational constant, reduce to unity.

If the rectangular coördinates of $P$ relative to rotating axes, taken along the line $S J$ and perpendicular to it at $O$, are $x$ and $y$ respectively (fig. $\mathrm{I}$ ), and if $t$ indicates

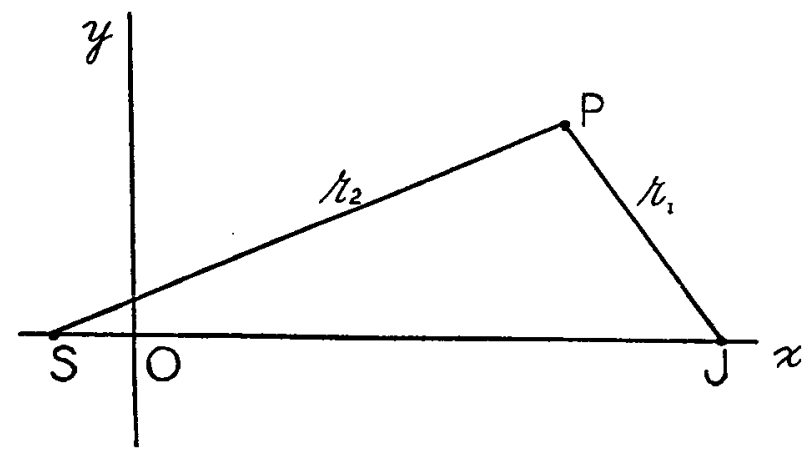

(Fig. 1).

the time, the differential equations of motion are

$$
\frac{d^{2} x}{d t^{2}}-2 \frac{d y}{d t}=\Omega_{x}(x, y), \quad \frac{d^{2} y}{d t^{2}}+2 \frac{d x}{d t}=\Omega_{y}(x, y),
$$

where

$$
\left\{\begin{aligned}
\Omega(x, y) & =\frac{\mathrm{I}}{2}\left[(\mathrm{I}-\mu) r_{\mathrm{I}}^{2}+\mu r_{2}^{2}\right]+\frac{\mathrm{I}-\mu}{r_{1}}+\frac{\mu}{r_{2}}, \\
r_{1} & =\sqrt{(x-\mu)^{2}+y^{2}}, \quad r_{2}=\sqrt{(x+\mathrm{I}-\mu)^{2}+y^{2}} .
\end{aligned}\right.
$$

In accordance with the notation for partial derivatives employed throughout the present paper, $\mathbf{\Omega}_{x}(x, y)$ and $\Omega_{y}(x, y)$ denote the partial derivatives of $\Omega(x, y)$ as to $x$ and $y$ respectively.

By multiplying the first and second equations (I) by $2 d x / d t$ and $2 d y / d t$ respec- 
tively, adding and integrating, we obtain the integral of JACOBI

$$
\left(\frac{d s}{d t}\right)^{2}=\left(\frac{d x}{d t}\right)^{2}+\left(\frac{d y}{d t}\right)^{2}=2 \Omega(x, y)-C
$$

where $s$ measures the arc length along the orbit of $P$, and $C$ is the constant of JACoBI.

In what follows we consider invariably the totality of motions of $P$ for which the constant $C$ has a given value. This specialization amounts to a use of the integral of JACOBI to reduce the system of differential equations ( $I$ ) from the fourth order to the third.

A simple way of accomplishing this reduction depends on the introduction of the new variable

$$
\varphi=\arctan \frac{d y}{d x}
$$

representing the angle between the direction of motion at any instant and the direction of the positive $x$-axis. We obtain at once from (I) and (3)

$$
\left\{\begin{array}{l}
\frac{d x}{d t}=\sqrt{2 \Omega(x, y)-C} \cos \varphi=X(x, y, \varphi), \\
\frac{d y}{d t}=\sqrt{2 \Omega(x, y)-C} \sin \varphi=Y(x, y, \varphi), \\
\frac{d \varphi}{d t}=-2+\frac{-\Omega_{x}(x, y) \sin \varphi+\Omega_{y}(x, y) \cos \varphi}{\sqrt{2 \Omega(x, y)-C}}=\Phi(x, y, \varphi) .
\end{array}\right.
$$

The first two equations result from the equations $d x / d s=\cos \varphi, d y / d s=\sin \varphi$, and from (3). The last equation may be deduced by forming $d \uparrow / d t$, and eliminating $d x / d t$, $d y / d t, d^{2} x / d t^{2}, d^{2} y / d t^{2}$ by the aid of the first two equations (5) and the equations (I). The equations ( 5 ) are equivalent to the equations (I) joined with the integral (3), and form a system of the third order.

If we observe that the right-hand members of the equations $(s)$ do not involve the variable $t$, it becomes evident that this variable may be eliminated and a reduction to the second order effected. The resulting equations may be written

$$
\frac{d x}{X(x, y, \varphi)}=\frac{d y}{Y(x, y, \varphi)}=\frac{d p}{\Phi(x, y, \varphi)} .
$$

Therefore, if $x, y, \varphi$ be taken as the rectangular coördinates of a point in space, the totality of curves of motion under consideration will be represented by a family of curves, one passing through each point of the cylindrical region $\Omega(x, y)-\frac{1}{2} C \geqslant 0$ to which the motion is necessarily confined.

This representation is singular only when the velocity is zero or infinite. The first of these two possibilities takes place when $P$ is on the curve of zero velocity $\boldsymbol{\Omega}(x, y)-\frac{1}{2} C=0$, and does not correspond to any singularity of the motion. The second takes place when $P$ collides with $S$ or $J$.

As time varies, each point of $x, y, \varphi$-space moves with a velocity, dependent on 
position only, along its curve of motion. Thus the totality of motions will correspond precisely to the steady motion of a fluid, which is incompressible in virtue of the identity

$$
X_{x}(x, y, \varphi)+Y_{y}(x, y, \varphi)+\Phi_{\varphi}(x, y, \varphi)=0 .
$$

Although this representation gives us some idea of the nature of the problem, it is possible to eliminate entirely the singularities of the representation by a suitable choice of variables. Such an elimination will be effected in $\$$ s.

$$
§ 2 .
$$

\section{On the Transformation of Certain Differential Equations.}

Let us turn for a moment to the consideration of the type of differential system

$$
\frac{d^{2} x}{d t^{2}}-2 \lambda(x, y) \frac{d y}{d t}=U_{x}(x, y), \quad \frac{d^{2} y}{d t^{2}}+2 \lambda(x, y) \frac{d x}{d t}=U_{y}(x, y),
$$

which constitutes a generalization of (I). We shall regard $t$ as the time, and $x, y$ as the coördinates of a moving particle in the $x, y$-plane.

We consider the triply infinite set of solutions of this system for which

$$
\left(\frac{d s}{d t}\right)^{2}=\left(\frac{d x}{d t}\right)^{2}+\left(\frac{d y}{d t}\right)^{2}=2 U(x, y),
$$

where $s$ measures length of arc. If now we introduce the variable $\varphi$ defined as in (4) we obtain without difficulty

$$
\left\{\begin{array}{l}
\frac{d x}{d t}=\sqrt{2 U(x, y)} \cos \varphi, \\
\frac{d y}{d t}=\sqrt{2 U(x, y)} \sin \varphi \\
\frac{d \psi}{d t}=-2 \lambda(x, y)+\frac{-U_{x}(x, y) \sin \varphi+U_{y}(x, y) \cos \varphi}{\sqrt{2 U(x, y)}} .
\end{array}\right.
$$

The curvature $K$ of the orbit is $d \varphi / d s$, which may be expressed in terms of $x$, $y, \psi$ by the aid of $\left(3^{\prime}\right)$ and the last one of the equations $\left(5^{\prime}\right)$ :

$$
K=\frac{-2 \lambda(x, y)}{\sqrt{2 U(x, y)}}+\frac{-U_{x}(x, y) \sin \varphi+U_{y}(x, y) \cos \varphi}{2 U(x, y)} .
$$

This gives an intrinsic formulation of the characteristic geometrical property of the curves of motion.

Let $n$ be the distance measured along the normal to the curve of motion at $(x, y)$ so chosen that the directions along which $s$ and $n$ increase are oriented like the positive $x$ - and $y$-axes respectively. Since $\varphi$ denotes the angle between the instantaneous direction of motion and the positive $x$-axis, the angle between the instantaneous normal and the positive $x$-axis is $\varphi+\frac{1}{2} \pi$. Thus the normal derivative of any function $f(x, y)$ 
is $-f_{x}(x, y) \sin p+f_{y}(x, y) \cos \varphi$. Bearing this in mind we may formulate the following conclusion:

The totality of curves $x=x(t), y=y(t)$ defined by the equations $\left(\mathrm{I}^{\prime}\right),\left(3^{\prime}\right)$ is the same as that defined by the equation

$$
\frac{d \arctan \frac{d y}{d x}}{d s}=-\frac{2 \lambda(x, y)}{\sqrt{2 U(x, y)}}+\frac{\mathrm{I}}{2} \frac{\partial}{\partial n} \log U(x, y),
$$

where $s$ measures the arc of the curve and $n$ is the distance measured along the instantaneous normal.

To complete a proof of this statement we need merely to show that we may pass conversely from $(7)$ to $\left(I^{\prime}\right),\left(3^{\prime}\right)$. But if we write

$$
\varphi=\arctan \frac{d y}{d x}, \quad t=\int \frac{d s}{\sqrt{2 U(x, y)}},
$$

we deduce $\left(5^{\prime}\right)$ at once from $(7)$, and thence $\left(\mathrm{I}^{\prime}\right),\left(3^{\prime}\right)$.

Let us now consider the result of making a transformation from the $x, y$ - to the $u, v$-plane defined by an equation $z=f(w)$ where $z=x+\sqrt{-1} y, w=u+\sqrt{-1} v$, and where $f(w)$ is an analytic function of $w$.

To effect this transformation of the totality of curves $\left(I^{\prime}\right),\left(3^{\prime}\right)$ we first note the relation

$$
d x+\sqrt{-\mathrm{I}} d y=f^{\prime}(w)(d u+\sqrt{-\mathrm{I}} d v)
$$

in which $f^{\prime}(w)$ denotes the derivative of $f(w)$. From this follows

and also

$$
\arctan \frac{d y}{d x}=\arctan \frac{d v}{d u}+\arg f^{\prime}(w),
$$

$$
d s=\left|f^{\prime}(w)\right| d s_{\mathrm{r}}, \quad d n=\left|f^{\prime}(w)\right| d n_{\mathrm{s}},
$$

where $s_{1}$ and $n_{1}$ are measured along the transformed curve and along its instantaneous normal respectively. Here we make use of familiar properties of a conformal transformation $z=f(w)$.

Furthermore, inasmuch as $\log \left|f^{\prime}(w)\right|$ and $\arg f^{\prime}(w)$ are conjugate harmonic functions, we have

$$
\frac{\partial}{\partial s} \arg f^{\prime}\left(w^{\prime}\right)=-\frac{\partial}{\partial n} \log \left|f^{\prime}\left(w^{\prime}\right)\right|
$$

by the Cauchy-Riemann differential equations.

Making use of these relations we obtain from (7)

$$
\frac{d \arctan \frac{d v}{d u}}{d s_{1}}=\frac{-2\left[\lambda(x, y)\left|f^{\prime}(w)\right|^{2}\right]}{\sqrt{2 U(x, y)\left|f^{\prime}(w)\right|^{2}}}+\frac{1}{2} \frac{\partial}{\partial n_{1}}\left[\log U(x, y)\left|f^{\prime}(w)\right|^{2}\right] .
$$

This is the intrinsic equation of the transformed curves, and is precisely of the form (7), with $x, y, \lambda(x, y)$, and $U(x, y)$ replaced by $u, v, \lambda(x, y)\left|f^{\prime}(w)\right|^{2}$, and $U(x, y)$ $\left|f^{\prime}(w)\right|^{2}$ respectively. 
Accordingly, by the italicized principle above, the curves of motion, referred to the coördinates $u$ and $v$, satisfy the equations

$$
\left\{\begin{array}{l}
\frac{d^{2} u}{d \tau^{2}}-2 \lambda(x, y)\left|f^{\prime}(w)\right|^{2} \frac{d v}{d \tau}=\left[U(x, y)\left|f^{\prime}(w)\right|^{2}\right]_{u} \\
\frac{d^{2} v}{d \tau^{2}}+2 \lambda(x, y)\left|f^{\prime}(w)\right|^{2} \frac{d u}{d \tau}=\left[U(x, y)\left|f^{\prime}(w)\right|^{2}\right]_{v^{\prime}} \\
\left(\frac{d s_{1}}{d \tau}\right)^{2}=\left(\frac{d u}{d \tau}\right)^{2}+\left(\frac{d v}{d \tau}\right)^{2}=2 U(x, y)\left|f^{\prime}(w)\right|^{2}
\end{array}\right.
$$

Moreover, since $d s=\left|f^{\prime}(w)\right| d s_{1}$, we infer at once that

$$
\frac{d t}{d \tau}=\frac{\frac{d s_{\mathrm{I}}}{d \tau}}{\frac{d s}{d t}} \cdot \frac{d s}{d s_{\mathrm{I}}}=\left|f^{\prime}(w)\right|^{2}
$$

By the transformation of variables

$$
x+\sqrt{-\mathrm{I}} y=f(u+\sqrt{-\mathrm{I}} v), \quad d t=\left|f^{\prime}(w)\right|^{2} d \tau,
$$

the totality of curves $x=x(t), y=y(t)$ defined by the equations ( $\left.\mathrm{I}^{\prime}\right),\left(3^{\prime}\right)$ goes into the totality of curves defined by a similar set of equations (9) obtained from ( $\left.\mathrm{I}^{\prime}\right),\left(3^{\prime}\right)$ by replacing $x, y, t, \lambda(x, y), U(x, y)$ by $u, v, \tau, \lambda(x, y)\left|f^{\prime}(w)\right|^{2}, U(x, y)\left|f^{\prime}(w)\right|^{2}$ respectively.

\section{$\$ 3$.}

\section{The Equations of Normal and Tangential Displacement.}

As a first application of this method of transformation, I shall derive the equations of normal and tangential displacement for the restricted problem of three bodies ${ }^{9}$ ). These equations will be useful later in the paper.

Let $x(t), y(t)$ be a solution of (I), (3), and let us make a conformal transformation of the $x, y$-plane which takes the corresponding orbit into the real axis in the transformed plane in such a way as to preserve arc lengths along the orbit. This transformation has the explicit form

$$
z=f(w)=f_{1}(w)+\sqrt{-1} f_{2}(w), \quad\left(x(t)=f_{1}(s), y(t)=f_{2}(s)\right) .
$$

Owing to the fact that the transformation is conformal, normal derivatives along the given orbit are unaltered by it. For this reason we will write $w=s+1 \sqrt{-\mathrm{I}} n$.

The application of the method of ttansformation to equations (I), (3), for which $\lambda(x, y)=\mathrm{I}$ and $U(x, y)=\Omega(x, y)-\frac{1}{2} C$, with the above choice of $f(w)$, gives

9) Cf. Darwin, loc. cit. 5), pp. I33-I4I. 
the equations

where

$$
\begin{gathered}
\frac{d^{2} s}{d \tau^{2}}-2\left|f^{\prime}(w)\right|^{2} \frac{d n}{d \tau}=F_{s}(s, n), \quad \frac{d^{2} n}{d \tau^{2}}+2\left|f^{\prime}(w)\right|^{2} \frac{d s}{d \tau}=F_{n}(s, n), \\
\left(\frac{d s}{d \tau}\right)^{2}+\left(\frac{d n}{d \tau}\right)^{2}=2 F(s, n),
\end{gathered}
$$

$$
F(s, n)=\left[\Omega(x, y)-\frac{1}{2} C\right]\left|f^{\prime}(w)\right|^{2} .
$$

Along the curve of motion we have

$$
\left|f^{\prime}\left(w^{\prime}\right)\right|=\left|\frac{d z}{d w^{\prime}}\right|=\mathbf{I}
$$

so that $d t=d \tau$ along this curve. Moreover this curve corresponds to the solution

$$
s=s_{\mathrm{o}}(\tau), \quad n=n_{\mathrm{o}}=\mathrm{o}
$$

of the transformed equations.

Substituting in these values for $\left|f^{\prime}(w)\right|, \tau, s, n$, we obtain

$$
\frac{d^{2} s_{\mathrm{o}}}{d t^{2}}=F_{s}\left(s_{\mathrm{o}}, 0\right), \quad 2 \frac{d s_{\mathrm{o}}}{d t}=F_{n}\left(s_{\mathrm{o}}, \mathrm{o}\right), \quad \frac{d s_{\mathrm{o}}}{d t}=\sqrt{2 F\left(s_{\mathrm{o}}, 0\right)},
$$

from which we deduce the condition

$$
\frac{1}{2} F_{n}\left(s_{0}, 0\right)=\sqrt{2 F\left(s_{0}, 0\right)} .
$$

Suppose now that we consider a varied solution $s=s_{0}+\varepsilon \delta s, n=\varepsilon \delta n$ where $\varepsilon$ is a constant which is to approach zero. The limiting forms of $\delta s, \delta n$ as $\varepsilon$ approaches zero are obtained as the solutions of the equations

$$
\begin{gathered}
\frac{d^{2} \delta s}{d t^{2}}-2 \frac{d \delta n}{d t}=F_{s s}\left(s_{0}, 0\right) \delta s+\frac{2 F_{s}\left(s_{0}, o\right)}{\sqrt{2 F\left(s_{0}, o\right)}} \delta n \\
\frac{d^{2} \delta n}{d t^{2}}+2 \frac{d \delta s}{d t}+2 \sqrt{2 F\left(s_{\mathrm{o}}, \mathrm{o}\right)}\left(\frac{\partial}{\partial n}\left|f^{\prime}\left(s_{\mathrm{o}}\right)\right|^{2}\right) \delta n=\frac{2 F_{s}\left(s_{\mathrm{o}}, \mathrm{o}\right)}{\sqrt{2 F\left(s_{\mathrm{o}}, \mathrm{o}\right)}} \delta s+F_{n n}\left(s_{\mathrm{o}}, 0\right) \delta n, \\
\sqrt{2 F\left(s_{\mathrm{o}}, \mathrm{o}\right)} \frac{d \delta s}{d t}=F_{s}\left(s_{\mathrm{o}}, 0\right) \delta s+2 \sqrt{2 F\left(s_{0}, 0\right)} \delta n,
\end{gathered}
$$

deduced by the usual method of variation. Here we have made use of the equations noted above and also of the equation

derived directly from them.

$$
F_{s n}\left(s_{0}, 0\right)=\frac{2 F_{s}\left(s_{0}, 0\right)}{\sqrt{2 F\left(s_{0}, 0\right)}}
$$

The first of the three equations for $\delta s, \delta n$ may be obtained from the last by differentiation. This is to be expected in consequence of the fact that (I) and (3) are not independent.

By combination of the second and third equations we can eliminate $\delta s$. Thus 
there results the final form of the equations of displacement

$$
\left\{\begin{array}{c}
\frac{d^{2} \delta n}{d t^{2}}+\left[4+2 \sqrt{2 F\left(s_{0}, 0\right)} \frac{\partial}{\partial n}\left|f^{\prime}\left(s_{0}\right)\right|^{2}-F_{n n}\left(s_{0}, 0\right)\right] \delta n=0 \\
\frac{d \delta s}{d t}-\frac{F_{s}\left(s_{0}, 0\right)}{\sqrt{2 F\left(s_{0}, o\right)}} \delta s=2 \delta n
\end{array}\right.
$$

If we agree to let $R(\rho)$ denote the teal part of $\rho$, and to let $I(\rho)$ denote the coefficient of $\sqrt{-\mathrm{I}}$ in $\rho$, the functions $f(w)$ and $F(s, n)$ which enter are given explicitly by the equations

(I I) $f(w)=x(t)+\sqrt{-\mathrm{r}} y(t), \quad F(s, n)=\left[\Omega(R[f(w)], I[f(w)])-\frac{1}{2} C\right]\left|f^{\prime}(w)\right|^{2}$, where $t$ is the function of $w=s+\sqrt{-\mathrm{I}} n$ such that

$$
w=\int \sqrt{\left(\frac{d x(t)}{d t}\right)^{2}+\left(\frac{d y(t)}{d t}\right)^{2}} d t .
$$

Moreover $s_{0}$ is the arc length taken along the orbit.

The first and second equations (ro) may be termed the differential equations of normal and tangential displacement respectively along the orbit $x=x(t), y=y(t)$. Every slightly varied orbit yields a normal and tangential displacement which are represented to terms of higher order by $\varepsilon \delta n, \varepsilon \delta s$ where $\varepsilon$ is an infinitesimal; and conversely.

The precise relation of the equation of normal displacement to the orbits is essentially contained in the usual theorems of the Calculus of Variations. In fact it is well-known that the parametric problem of minimizing the integral

$$
\int\left\{\left(x \frac{d y}{d t}-y \frac{d x}{d t}\right)+\sqrt{2 \Omega(x, y)-C} \sqrt{\left(\frac{d x}{d t}\right)^{2}+\left(\frac{d y}{d t}\right)^{2}}\right\} d t
$$

leads at once to a set of extremals which are precisely the orbits in the restricted problem of three bodies for the given $C$. This becomes immediately obvious if the parameter $t$ is so chosen that the integral relation (3) holds. The equation of normal displacement is the differential equation of JACOBI for this Calculus of Variations problem.

$$
\$ 4 \text {. }
$$

Levi-Civita's Form of the Equations of Motion.

The form of ( 1 ), (3) due to Levi-Civita results from the following transformation of variables

$$
x-\mu=p^{2}-q^{2}, \quad y=2 p q, \quad d t=4\left(p^{2}+q^{2}\right) d \tau .
$$

This may be written

$$
\tilde{z}=f(w)=\mu+w^{2}, \quad d t=\left|f^{\prime}(w)\right|^{2} d \tau
$$

where $z=x+\sqrt{-\mathrm{I}} y, w=p+\sqrt{-\mathrm{I}} q$, and falls under the type of transformation 
considered in $\ 2$. If applied to (I), (3) we obtain at once essentially the equations of Levi-Civita

$$
\left\{\begin{aligned}
\frac{d^{2} p}{d \tau^{2}}-8\left(p^{2}+q^{2}\right) \frac{d q}{d \tau} & =\left[4\left(\Omega\left(p^{2}-q^{2}, 2 p q\right)-\frac{\mathrm{I}}{2} C\right)\left(p^{2}+q^{2}\right)\right]_{p} \\
\frac{d^{2} q}{d \tau^{2}}+8\left(p^{2}+q^{2}\right) \frac{d p}{d \tau} & =\left[4\left(\Omega\left(p^{2}-q^{2}, 2 p q\right)-\frac{\mathrm{I}}{2} C\right)\left(p^{2}+q^{2}\right)\right]_{q} \\
\left(\frac{d p}{d \tau}\right)^{2}+\left(\frac{d q}{d \tau}\right)^{2} & =8\left(\Omega\left(p^{2}-q^{2}, 2 p q\right)-\frac{\mathrm{I}}{2} C\right)\left(p^{2}+q^{2}\right)
\end{aligned}\right.
$$

in accordance with the results of $\ 2$.

By this transformation the point $J$ goes into the origin in the $p$, $q$-plane, which is not a singular point. In fact the term $(1-\mu) / r_{1}$ or $(1-\mu) /\left(p^{2}+q^{2}\right)$ in $\Omega$, which becomes infinite, appears multiplied by a factor $\left(p^{2}+q^{2}\right)$.

At $p=q=0$ the third equation ( 14 ) shows that the square of the velocity is $8(1-\mu)$ for the possible orbits. Hence the curves of motion are analytic curves without singularity near the origin.

In consequence of these facts it is apparent that the orbits approach a cusped form near collision with $S$, and likewise with $J^{\mathrm{I}}$ ).

The singularities of the transformed equations lie at $\infty$ and at the two images of the point $S$ in the $p, q$-plane.

\section{S.}

\section{A New Form of the Equations of Motion.}

In the present paragraph we shall use a similar transformation to remove the singularities at both $S$ and $J$ [see ${ }^{\mathbf{I}}$ ), Note]. The resultant form of equations yields essential aid in Part II, and is undoubtedly of definite theoretical interest. Nevertheless, in Parts III and IV of the present paper, the simpler equations of LEvi-Civita turn out to be more convenient.

In order to completely regularize the equations of motion, we proceed to effect the transformation of ( $\mathrm{I}),(3)$ corresponding to

$$
z=f(w)=\frac{w^{2}+\mu(1-\mu)}{2 w+1-2 \mu} .
$$

The transformation from $z$ to $u$ admits of the alternative form

$$
\frac{z+I-\mu}{z-\mu}=\left(\frac{w+I-\mu}{w-\mu}\right)^{2} \text {. }
$$

From the equation (IS) we find at once

$$
z-\mu=\frac{(w-\mu)^{2}}{2 w+1-2 \mu}, \quad z+1-\mu=\frac{(w+1-\mu)^{2}}{2 w+1-2 \mu},
$$


and also

$$
f^{\prime}(w)=\frac{2(w-\mu)(w+I-\mu)}{(2 w+1-2 \mu)^{2}} .
$$

The explicit transformation from $x, y, l$ to $u, v, \tau$ is $(\mathbb{S} 2)$

(I9)

$$
\left\{\begin{array}{l}
x=\frac{1}{2}\left(u-\frac{1}{2}+\mu\right)+\frac{1}{8} \frac{u+\frac{1}{2}-\mu}{\left(u+\frac{1}{2}-\mu\right)^{2}+v^{2}}, \\
y=\frac{1}{2} v-\frac{1}{8} \frac{v}{\left(u+\frac{1}{2}-\mu\right)^{2}+v^{2}}, \\
d t=\frac{\left[(u-\mu)^{2}+v^{2}\right]\left[(u+1-\mu)^{2}+v^{2}\right]}{4\left[\left(u+\frac{1}{2}-u\right)^{2}+v^{2}\right]^{4}} d \tau .
\end{array}\right.
$$

The resultant form of equations is then

where

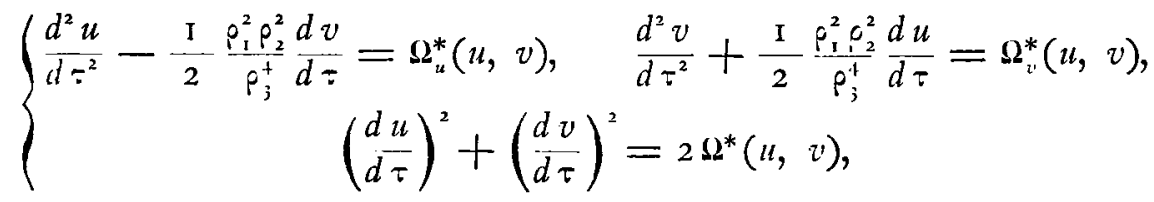

$$
\Omega^{*}(u, v)=\frac{\rho_{1}^{2} \rho_{2}^{2}}{4 \rho_{3}^{4}}\left[\frac{(I-\mu) p_{1}^{4}+\mu_{i} i_{2}^{4}}{8 \rho_{3}^{2}}+2 \rho_{3}\left(\frac{\mu}{\rho_{1}^{2}}+\frac{I-\psi}{\rho_{2}^{2}}\right)-\frac{I}{2} C\right],
$$

$$
p_{1}=\sqrt{(u-\mu)^{2}+v^{2}}, \quad p_{2}=\sqrt{(u+I-\mu)^{2}+v^{2}}, \quad \rho_{3}=\sqrt{\left(u+\frac{1}{2}-\mu\right)^{2}+v^{2}} .
$$

To obtain these equations we apply the method of $₫ 2$ for $\lambda(x, y)=\mathrm{I}$ and $U(x, y)=\mathbf{\Omega}(x, y)-\frac{\mathrm{t}}{2} C$; we need only express $\left|f^{\prime}(w)\right|$ and $\boldsymbol{\Omega}(x, y)-\frac{1}{2} C$ in terms of the new variables $u, v$, and substitute these values together with $\lambda(x, y)=\mathrm{I}$ in equation (9). By (I8) we find

Also by (17) we find

$$
\left|f^{\prime}(w)\right|=\frac{p_{1} p_{2}}{2 p_{3}^{2}}
$$

$$
r_{1}=|z-\mu|=\frac{p_{1}^{2}}{2 p_{3}}, \quad r_{2}=|z+I-\mu|=\frac{p_{2}^{2}}{2 p_{3}} .
$$

From (2) and these last equations we derive

$$
\Omega(x, y)-\frac{\mathrm{I}}{2} C=\frac{\mathrm{I}}{2} \frac{(\mathrm{I}-\mu) \rho_{1}^{4}+\mu \rho_{2}^{4}}{4 \rho_{3}^{2}}+2 \rho_{3}\left(\frac{\mathrm{I}-\mu}{\rho_{1}^{2}}+\frac{\mu}{\rho_{2}^{2}}\right)-\frac{\mathrm{I}}{2} C .
$$

The points $w=\mu$ and $w=-\mathrm{I}+\mu$ correspond to the points $z=\mu$ and $z=-\mathbf{I}+\mu$ respectively. Now $\Omega^{*}(u, v)$ is analytic at these two points in the $u$, $v$-plane. The coefficients of $d u / d \tau$ and $d v / d \tau$ in the equations (2o) are also analytic. Thus the new equations have an ordinary point at $(\mu, 0)$ and $(-I+\mu, 0)$ in the $u, v$-plane corresponding to $(\mu, 0)$ and $(-\mathrm{I}+u, 0)$ in the $x, y$-plane. The nature of the solutions at these points is given by the customary fundamental existence theorems for ordinary differential equations.

The same examination shows that the only finite singular point of the new equa- 
tions is at $\left(-\frac{1}{2}+\mu, 0\right)$ when $p_{3}$ is zero. This point $w=-\frac{1}{2}+\mu$, and also $w=\infty$, correspond to $z=\infty$.

The equations (20) remain regular so long as the particle $P$ is not rejected to infinity in the $x$, y-plane.

The importance of the new form of equations consists in the fact that $u, v$, $u^{\prime}=d u / d \tau, v^{\prime}=d v / d \tau$ form an adequate set of coördinates of the motion under all circumstances. Any set of values $u, v, u^{\prime}$, $v^{\prime}$ satisfying the relation

$$
u^{\prime 2}+v^{\prime 2}-2 \Omega^{*}(u, v)=0
$$

will be termed a state of motion.

For each state of motion we have a unique set of values $x, y, d x / d t, d y / d t$ satisfying (3) provided that the point $(x, y)$ does not coincide with $S$ or $J$. In these exceptional cases it has been noted $(\$ 4)$ that the motion is represented by a cusped orbit in the $x, y$-plane with cusp at $S$ or $J$. To each $u^{\prime}, v^{\prime}$ corresponds a possible direction of the axis of the cusp.

We shall agree to refer the state of motion to the $x, y$ plane in which the particle moves, and we shall proceed to establish that for a given state of motion there exist alway's two and only two distinct sets of values $u, v, u^{\prime}, v^{\prime}$.

If we denote by $w_{1}$ and $w_{2}$ the two values of $w$ for a given $z$, there subsists between these values the relation

$$
2-\frac{\mathrm{I}}{w_{1}+\mathrm{I}-\mu}-\frac{\mathrm{I}}{w_{2}+\mathrm{I}-\mu_{0}}=0 .
$$

This relation may be obtained directly from (16). The transformations from $z$ to $w_{1}$ and $w_{2}$ are analytic.

Let $\tau_{1}$ and $\tau_{2}$ be the variables $\tau$ corresponding to $w_{1}$ and $w_{2}$, derived by the method given in $\ 2$. In accordance with our previous notation it is natural to write

and also

$$
w_{1}=u_{1}+\sqrt{-\mathrm{I}} v_{1}, \quad w_{2}=u_{2}+\sqrt{-\mathrm{I}} v_{2}
$$

$$
d w_{1} / d \tau_{1}=u_{1}^{\prime}+\sqrt{-\mathrm{I}} v_{1}^{\prime}, \quad d w_{2} / d \tau_{2}=u_{2}^{\prime}+\sqrt{-\mathrm{I}} v_{2}^{\prime} .
$$

These transformations from $z$ to $w_{1}$ and $w_{2}$ lead us of course to the equations (20), (2I) in which $u, v, \tau$ are replaced by $u_{1}, v_{1}, \tau_{1}$ and $u_{2}, v_{2}, \tau_{2}$ respectively. The relation between $\tau_{1}$ and $\tau_{2}$ is furnished by the equations

But we have identically

$$
d t=\left|f^{\prime}\left(w_{1}\right)\right|^{2} d \tau_{1}=\left|f^{\prime}\left(w_{2}\right)\right|^{2} d \tau_{2} .
$$

so that

and thus we find

$$
z=f\left(w_{1}\right)=f\left(w_{2}\right)
$$

where

$$
f^{\prime}\left(w_{1}\right) d w_{1}=f^{\prime}\left(w_{2}\right) d w_{2},
$$

$$
\frac{f^{\prime}\left(w_{1}\right) w_{1}^{\prime}}{\left|f^{\prime}\left(w_{1}\right)\right|^{2}}=\frac{f^{\prime}\left(w_{2}\right) w_{2}^{\prime}}{\left|f^{\prime}\left(w_{2}\right)\right|^{2}}
$$

$$
w_{1}^{\prime}=d w_{1} / d \tau_{1}, \quad w_{2}^{\prime}=d w_{2} / d \tau_{2} .
$$


The equations (23) and (24) give explicitly the relation between the two sets $u_{1}, v_{1}, u_{1}^{\prime}, v_{1}^{\prime}$ and $u_{2}, v_{2}, u_{2}^{\prime}, v_{2}^{\prime}$ which correspond to the same state of motion.

It is evident that these sets are distinct as long as $w_{1}$ and $w_{2}$ are distinct. Now $w_{1}$ and $w_{2}$ only coincide at $\mu$ or - I $+\mu$. If $w_{1}$ approaches $w_{2}$ in this way we obtain

from (23). Hence we infer that

$$
\lim \frac{f^{\prime}\left(w_{1}\right)}{f^{\prime}\left(w_{2}\right)}=\lim \frac{d w_{2}}{d w_{1}}=-I
$$

$$
w_{1}^{\prime}+w_{2}^{\prime}=0
$$

If $w_{1}^{\prime}$ and $w_{2}^{\prime}$ were equal they would reduce to zero for $w_{1}=w_{2}=\mu$ or $-\mathrm{I}+\mu$, in contradiction to (22). This establishes that the two sets are distinct in all cases.

We note in passing that the analytic transformation from $w_{1}$ to $w_{2}$ is of the type considered in $\ 2$. It may be verified without difficulty that this transformation takes the equations (20) into themselves. At the same time it may be observed that the non-analytic transformation which replaces $u, v, \tau$ by $u,-v,-\tau$ also leaves the equations invariant in form.

PART II.

ANALYSIS SITUS OF THE MANIFOLDS OF STATES OF MOTION.

$$
\$ 6 .
$$

Geometric Representation.

We are now in a position to form a satisfactory geometric representation of the totality of motions given by (I), (3). Conceive of $u, v, u^{\prime}, v^{\prime}$ as the rectangular coördinates of a four-dimensional space, and consider the three-dimensional manifold or manifolds (22) lying in that space. These manifolds comprise all of the points representative of possible states of motion, and no others.

The three-dimensional manifolds of states of motion are without singularity unless $C$ bas one of five particular values.

In fact, since the left-hand member of (22) is analytic for any conceivable set of such values, it follows that the equations for a singularity are

$$
u^{\prime}=\mathrm{o}, \quad v^{\prime}=\mathrm{o}, \quad \Omega_{u}^{*}(u, v)=\mathrm{o}, \quad \mathbf{\Omega}_{v}^{*}(u, v)=\mathrm{o} .
$$

The first two equations imply that $\Omega(x, y)-\frac{r}{2} C$ is zero at the corresponding point $(x, y)$, 
since the function $\Omega^{*}(u, v)$ does not vanish at $S$ or $J$ and can vanish elsewhere only when $\mathbf{\Omega}(x, y)-\frac{1}{2} C$ vanishes. The last two equations imply that $\mathbf{\Omega}_{\mathrm{r}}(x, y)=\mathbf{\Omega}_{\mathrm{y}}(x, y)=0$.

These last equations may be written [see (2)]

$$
\left\{\begin{array}{l}
\Omega_{x}(x, y) \equiv x-\frac{(\mathrm{I}-\mu)(x-\mu)}{r_{1}^{3}}-\frac{\mu(x+\mathrm{I}-\mu)}{r_{2}^{3}}=0, \\
\Omega_{y}(x, y) \equiv y\left(\mathrm{I}-\frac{\mathrm{I}-\mu}{r_{1}^{3}}-\frac{\mu}{r_{2}^{3}}\right)=0 .
\end{array}\right.
$$

It is verified without difficulty that there are five solutions $(x, y)$ :

(27) $\left\{\begin{array}{l}P_{1} \equiv\left(x_{1}, 0\right), \quad P_{2} \equiv\left(x_{2}, 0\right), \quad P_{3} \equiv\left(x_{3}, 0\right) \quad\left(x_{1}<-1+\mu<x_{2}<\mu<x_{5}\right), \\ \left.P_{4} \equiv\left(-\frac{1}{2}+\mu, \frac{1}{2} \sqrt{3}\right), \quad P_{5} \equiv\left(-\frac{1}{2}+\mu,-\frac{1}{2} \sqrt{3}\right)^{\text {Io }}\right) .\end{array}\right.$

Thus there is a singularity of such a manifold only when $C$ has one of the five values necessary to make $\Omega(x, y)-\frac{1}{2} C$ vanish at $P_{1}, P_{2}, P_{3}, P_{4}$ or $P_{5}$. At these values, for the corresponding $u, v$ and for $u^{\prime}=v^{\prime}=0$, two of the manifolds (22) are about to unite or separate. We shall exclude these values of $C$ from consideration, so that in all remaining cases the manifold is non-singular and any small part of it may be represented in ordinary space.

To each point of a manifold of states of motion there will correspond one and only one curve of motion. This will pass through the point with a definite direction which is an analytic function of position in the space.

In fact, we have from (20)

$$
\frac{d u}{u^{\prime}}=\frac{d v}{v^{\prime}}=\frac{d u^{\prime}}{\frac{1}{2} \rho_{1}^{2} \rho_{2}^{2} \rho_{j}^{-1} v^{\prime}+\mathbf{\Omega}_{u}^{*}(u, v)}=\frac{d v^{\prime}}{-\frac{1}{2} \rho_{1}^{2} \rho_{2}^{2} i_{j}^{-+} u^{\prime}+\Omega_{v}^{*}(u, v)} .
$$

Our statement is certainly valid unless all four denominators can vanish simultaneously. In this case four equations, essentially the same as the four equations for a singularity of the manifold, are obtained. These have been proved inconsistent unless ${ }^{\circ} C$ has one of the five excluded values.

The totality of states of motion may thus be thought of as given by the steady motion of a three-dimensional flow occupying a non-singular manifold in four-dimensional space. As $\mathrm{r}$ changes, each point moves along a non-singular stream line which represents the orbit in the $x, y$-plane.

This motion leaves unaltered the volume integral

$$
\iiint \frac{d u d v\left(u^{\prime} d v^{\prime}-v^{\prime} d u^{\prime}\right)}{2 \Omega^{*}(u, v)}
$$

extended over a part or the whole of the three-dimensional manifold (22). For, if we write $\sqcup=\arctan d v / d u$ and employ (22), it is clear that the above integral becomes

$$
\iiint d u d v d \psi
$$

Io) CF. C. L. Charlier, Die Mechanik des Himmels (Leipzig, Veit, 1907), vol. II, pp. Ioz-I I7. 
Now our equations of motion (20) may be written [compare with $\left(5^{\prime}\right)$ ]

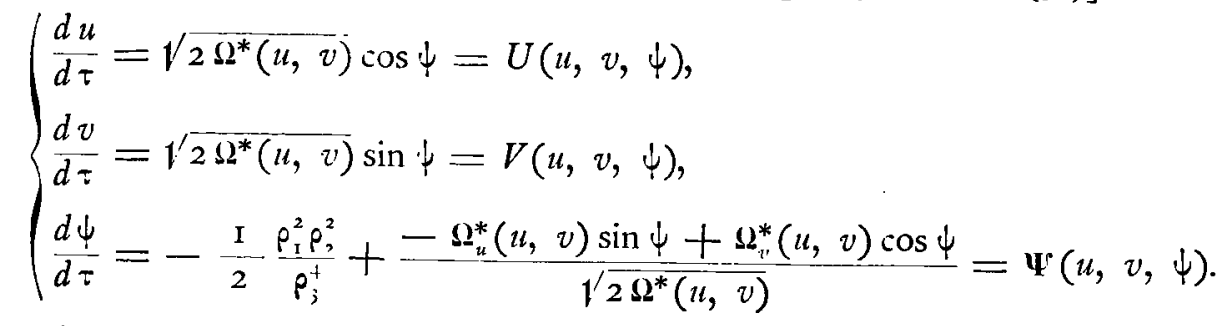

But we have

$$
U_{u}(u, v, \psi)+V_{v}(u, v, \psi)+\Psi_{\psi}(u, v, \psi) \equiv \mathrm{o}
$$

so that the integral (29) does remain invariant.

\section{$\int 7$.}

\section{On the Regions of Motion.}

It is of primary importance to determine the nature of the three-dimensional manifolds of states of motion. Prior to doing this we need to consider the nature of the regions $\Omega(x, y)-\frac{1}{2} C \supseteq 0$ in the $x, y$-plane, and of the corresponding regions $\Omega^{*}(u, v) \gg 0$ in the $u, v$-plane in which, by (3) and the last equation (20), motion is only possible. The essential facts concerning the regions in the $x, y$-plane are wellknown and will only be outlined.

We have observed [see (26) and (27)] that the simultaneous equations $\Omega_{x}(x, y)=0$, $\underline{\Omega}(x, y)=\mathrm{o}$ are satisfied at five points $P_{1}, P_{2}, P_{;}, P_{+}, P_{5}$ of the $x, y$-plane. The first three of these lie on the axis of $x$ on the three segments into which $S$ and $J$ divide that axis. The last two points form the remaining vertex of the two equilateral triangles which have $S$ and $J$ as vertices. Also $\Omega(x, y)$ becomes infinite at $S$, at $J$, and at infinity, and is everywhere positive.

Consider the family of algebraic curves $\Omega(x, y)-\frac{1}{2} C=0$ which form the boundaries of the regions under consideration. For each pair of points $(x, y),(x,-y)$ we get one and only one value of $C$. Therefore if $C$ be made to decrease from $+\infty$ to zero, each member of the family of curves generated is symmetric with respect to the $x$-axis, and one of the curves passes through each point of the $x, y$-plane.

For $C$ large and positive we obtain obviously two small approximately circular ovals about $S$ and $J$, and a large approximately circular oval containing $S$ and $J$. For such values of the constant $C$ the motion takes place either within one of the smaller ovals (Case I) or outside of the large oval containing $S$ and $J$ (Case II), inasmuch as $\mathbf{\Omega}(x, y)-\frac{1}{2} C$ must be positive by (3).

As $C$ diminishes, the small ovals expand and the large one contracts until the two small ovals unite at $P_{2}$ along the axis.

In fact, it can be established without difficulty that at $P_{4}$ and $P_{5}$ the function 
$\boldsymbol{\Omega}(x, y)$ has its absolute minimum. It can also be established that the absolute minimum of $\mathbf{\Omega}(x, y)$ within the segment $S J$ of the $x$-axis exceeds the value of $\mathbf{\Omega}(x, y)$ at the same distance from $S$ or $J$ but outside of this segment. Consequently $\Omega(x, y)$ has a greater value at $P_{2}$ than at $P_{1}, P_{3}, P_{4}, P_{5}$, and the two smaller ovals will unite at $P_{2}$ as stated.

Within the double oval about $S$ and $J$, so formed, motion may take place (Case III),

At the next stage the large outer oval and the double oval unite at $P_{1}$ (or $P_{3}$ ) into a horse-shoe shaped oval cutting the $x$-axis twice to the right of $J$ (or the left of $S$ ). Motion may take place outside of this oval (Case IV).

If $C$ be decreased further, this oval breaks up into two symmetrical ones on opposite sides of the $x$-axis by passage through $P_{3}$ ( or $P_{1}$ ), and motion takes place outside of these two ovals (Case V).

These two ovals diminish to the points $P_{4}, P_{5}$ and vanish, as $C$ decreases further to 3. For $C<3$ motion is possible throughout the plane (Case VI).

These are the well-known facts which we shall need to use ${ }^{\text {Ir }}$ ).

Let us now inquire into the nature of these regions of motion as represented in the $u, v$-plane. The transformation from the $x, y$-plane to the $u, v$-plane is given by (16). This equation shows that a suitable representation of the transformation may be obtained by taking a RIEMAN surface of two leaves in the $x$, $y^{\prime}$-plane with branch points at $S$ and $J$, and making one value of $w$ correspond to each point of this surface.

It may be observed at once that the ovals in the $u$, $v$-plane are symmetric with respect to the real axis, inasmuch as the ovals in the $x, y$-plane are symmetric with respect to the axis of reals in that plane, and conjugate values of $z$ yield conjugate pairs of values of $w$.

In Case I the region of motion including $J$ may be deformed into a small oval about $J$ in this Riemans surface without passing over the branch points $S$ and $J$. Hence in the $u, v$-plane the region of possible motion will be the interior of an oval about $w=\mu$.

In Case II the region of motion may be deformed (under the same restriction) into the exterior of two superimposed large ovals about $z=\infty$ including $S$ and $J$, one in each leaf of the RIEMANn surface. These regions correspond to the interior of an oval about $w=\frac{1}{2}-\mu$ and to the exterior of an oval about $w=\infty$ in the $u$, $v$-plane. The motion may be represented in either region.

In Case III the region of motion may be deformed into the interior of two large superimposed ovals about $z=\infty$ in the RiEmany surface. In the $u, v$-plane the region of motion will accordingly consist of the ring lying between an oval about $w=-\frac{1}{2}+\mu$ and an oval about $w=\infty$. The points $w=-I+\mu$ and $w=\mu$. lie one on each of the two segments of the $u$-axis within the ring.

I I) Loc. cit. Io). 
In Case IV we have two superimposed ovals in the RIEmann surface, each of which can be deformed to a point. Hence in the $u$, $v$-plane there will be two ovals. Since, at the two intersections of the oral with the $x$-axis in the $x$, $y$-plane, the ratio $(z+1-\mu) /(z-\mu)$ is real and positive, each of the ovals in the $u$, $v$-plane intersects the real axis twice. The fraction $(w+I-\mu) /(w-\mu)$ is positive for one oval and negative for the other; the first oval cuts the $u$-axis to the left of $w=-1+u$, or to the right of $w=\mu$, and the second oval cuts the $u$-axis between $w=-I+\mu$ and $w=\mu$. on the same side of $w=-\frac{1}{2}+\mu$. The region of possible motion is the region exterior to these ovals.

In Case $V$ the two ovals in the $x, y$-plane will yield four ovals superimposed in pairs in the RIEMANN surface, all of which can be deformed to a point. The region of motion in the $u, v$-plane will therefore be the part of the plane exterior to four ovals. Inasmuch as the ovals in the $x, y$-plane do not cut the real axis, the ovals in the $u$, $v$-plane will not cut the real axis, and the four ovals are two-by-two symmetrically placed with respect to that axis.

In Case VI motion is possible throughout the $u$, v-plane.

\section{$\$ 8$.}

\section{A Problem in Analysis Situs.}

We are now in a position to consider the nature of the three-dimensional manifolds of states of motion, and we begin with Case I. The point $(u, v)$ is restricted to lie in an oval about $J$ in the $u$, v-plane. Any such pair of values $u$, $v$ together with values $u^{\prime}, v^{\prime}$ subject to (22) yields a possible state of motion.

But to any point $\left(u_{1}, v_{1}\right)$ in this oval there is a point $\left(u_{2}, v_{2}\right)$, also within it, such that the two associated points in the RIEMAnN surface given by (16) lie one above the other. The corresponding values $w_{1}^{\prime}$ and $w_{2}$ of $w$ are related by (23). This equation makes it certain either that one of the points $\left(u_{1}, v_{1}\right),\left(u_{2}, v_{2}\right)$ lies in the upper half plane and the other in the lower half plane, or else that both lie along the real axis.

Let us therefore confine ourselves to the upper half plane $v \gg 0$. By doing so we obtain a single representation of each state of motion for $v \neq 0$. A double representation of each state of motion $v=0$ will be obtained.

Now, given $u, v \geqslant 0$, and $u^{\prime}$ such that $\left|u^{\prime}\right| \leq \sqrt{2 \Omega^{*}(u, v)}$, there will correspond two values of $v^{\prime}$ equal in magnitude and opposite in sign such that $u, v, u^{\prime}, v^{\prime}$ satisfy (22). These values of $v^{\prime}$ coincide and become zero for $\left|u^{\prime}\right|=\sqrt{2 \Omega^{*}(u, v)}$.

Thus the manifold in question $(v \gg 0)$ is in two-to-one continuous correspondence with the three-dimensional manifold

$$
2 \Omega^{*}(u, v) \supseteq 0, \quad v \supseteq 0, \quad-\sqrt{2 \Omega^{*}} \overline{(u, v)} \leqslant u^{\prime} \leq \sqrt{2 \Omega^{*}(u, v)}
$$

The two values coincide along the surface $\left|u^{\prime}\right|=\sqrt{2 \Omega^{*}(u, v)}$. 
Taking into account the fact that $\sqrt{2 \Omega^{*}(u, v)}$ is positive within the oval and zero along its boundary, we see that the above $u$, $v$, $u^{\prime}$-manifold is in one-to-one continuous correspondence with the $\bar{u}, \bar{v}, \overline{u^{\prime}}$-manifold

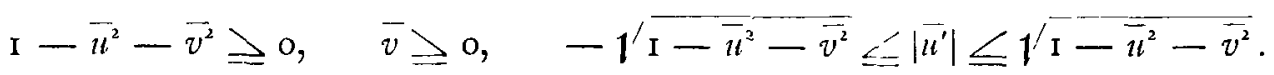

If $\bar{u}, \bar{v}, \bar{u}$ ' be taken as rectangular coördinates of a point, this represents a hemisphere.

The manifold of states of motion which we are considering $(v \gg 0)$ and the points of this hemisphere, doubly taken except along the spherical surface, are in oneto-one continuous correspondence. The states of motion $\imath^{\prime}=0$ will be represented by points of the plane surface $\bar{v}=0$.

It follows that the states of motion in question are in one-to-one continuous correspondence with the points of a sphere. This sphere may be conceived of as formed from the two hemispheres by reflecting one of the hemispheres in its spherical boundary, leaving points of that boundary invariant, and by cffecting a suitable distortion.

The bounding surface of the sphere so obtained is compounded of two hemispherical parts coming from the doubly taken plane boundary of the hemisphere. These parts are in one-to-one continuous correspondence with the manifold $u, v=0, u$, $v^{\prime} \supseteq \mathrm{o}$ and the manifold $u, v^{\prime}=0, u^{\prime}, v^{\prime} \leqslant \mathrm{o}$ respectively, and have in common the bounding circle for which $v^{\prime}=0$.

If we employ the notation of $\$ 5$ to represent the pair of points $\left(u, v, u^{\prime}, v^{\prime}\right)$ giving the same state of motion, it is clear that $w_{1}$ and $w_{2}$ are real for $v=0$; also from (23) it appears that the ratio of $w_{1}^{\prime}$ to $w_{2}^{\prime}$ is real and negative in this case. Hence each point of one part of the bounding surface is paired with a point of the other part of the surface which represents the same state of motion. Along the common circle, a point $u_{1}, v_{1}=0, u i_{1}^{\prime}, v_{1}^{\prime}=0$ will be paired with a point $u_{2}, v_{2}=0$, $u_{2}^{\prime}, v_{2}^{\prime}=0$ of the same boundary, and no pair of points coincide in one (see $\$ 5$ ). This property shows that the pairing along the boundary is essentially equivalent to that obtained by pairing opposite points of the circle and may be reduced to it by distortion.

By a second distortion each point in one bounding hemispherical surface of the sphere may be made to lie diametrically opposite the point with which it is paired in the other hemispherical surface.

In Case I, when the motion takes place in an oval of zero velocity about $J$, the states of motion are in one-to-one continuous correspondence with the points of a spbere on which opposite points are taken as identical.

Let $x_{1}^{2}+x_{2}^{2}+x_{3}^{2}=\mathrm{I}$ be the equation of this sphere, where $x_{1}, x_{2}, x_{3}$ are rectangular coördinates; if then we write

$$
\bar{x}_{1}=\frac{-x_{1}}{\sqrt{x_{1}^{2}+x_{2}^{2}+x_{3}^{2}}}, \quad \bar{x}_{2}=\frac{-x_{2}}{\sqrt{x_{1}^{2}+x_{2}^{2}+x_{3}^{2}}}, \quad \bar{x}_{3}=\frac{-x_{3}}{\sqrt{x_{1}^{2}+x_{2}^{2}+x_{3}^{2}}},
$$

the points $x_{1}, x_{2}, x_{3}$ and $\bar{x}_{1}, \bar{x}_{2}, \bar{x}_{3}$ of the space of inversion are so paired that the 
interior of the sphere corresponds to the exterior of the sphere, and opposite points on the sphere are paired. It follows that the manifold of states of motion in Case I is in twoto one continuous correspondence with the points of the space of inversion ${ }^{2}$ ).

In Case II it has been noted that for any point in the oval in the $x, y$-plane there is but one point of the oval about $v=-\frac{1}{2}+v$ in the $u$, $v$-plane. Moreover the point $w=-\frac{1}{2}+\mu$. corresponds to the singular point $z=\infty$. By (22) the manifold of states of motion will be in one-to-one continuous correspondence with a manifold

$$
\bar{u}^{\prime 2}+\bar{v}^{\prime 2}=\mathrm{I}-\bar{u}^{2}-\bar{v}^{2}
$$

from which the sets of values $\bar{u}=\bar{v}=0$, corresponding to $w=-\frac{1}{2}+\mu$, are excluded. But this is the surface of a hypersphere in four-dimensional space from which the closed line $\bar{u}=\bar{v}=0, \bar{u}^{\prime 2}+{\overline{z^{\prime}}}^{2}=\mathrm{I}$ is excluded. Either this fact or a direct analysis of the $\bar{u}, \bar{v}, \bar{u}, \bar{v}^{\prime}$-manifold leads to the conclusion:

In Case II, when the motion takes place in the exterior of an oval including $S$ and $J$, the stat's of motion are in one-to.une continuous correspontence with the points of the space of inversion exclusive of a closed singular line whose neighborbood corresponds to the neigbborbood of infinity in the $x, y$-plane.

By an expansion of the singular line into a closed tube and an inversion of space about an interior point of the tube it is possible to obtain a representation in the interior of an anchor ring whose boundary corresponds to the neighborhood of infinity.

It would have been possible to obtain the results for Case I somewhat more simply from the equations of Levi-Civita, and for Case II from the equations (I).

To deal with Case III we proceed much as in Case I. As in Case I we have two points in the three-dimensional manifold $u, v, u^{\prime}, v^{\prime}$ representative of each state of motion. This appears from the fact that superimposed points within the double oval in the RIEMANN surface correspond to distinct points in the single ring-shaped region in the $u$, v-plane.

Moreover, as before, we obtain a single representation of each state of motion by taking $v \gg 0$, with the exception of the states of motion $v=0$, which are doubly represented. For a given $u, v \gg 0$ and $u^{\prime}$ such that $\left|u^{\prime}\right| \leq \sqrt{2 \Omega^{*}(u, v)}$, we have two values of $v^{\prime}$ equal in magnitude but opposite in sign. These values merge and become zero for $\left|u^{\prime}\right|=\sqrt{2 \Omega^{*}(u, v)}$.

The function $\Omega^{*}(u, v)$ is positive within the ring-shaped region of motion in the $u, v$-plans, and vanishes along the two boundaries. Let $u, v, u^{\prime}$ be taken as rectangular coördinates of a point. If we bear in mind the condition $v \geqslant 0$, it is clear that the states of motion under consideration are in two-to-one continuous correspondence

12) Cf. H. Poincaré, loc. cit. ${ }^{2}$ ), p. 199. Also ${ }^{3}$ ), p. 380 . Professor O. Veblen has called my attention to the fact that the space of projective geometry also affords an equivalent representing space. This is not, however, the space of the coördinates. 


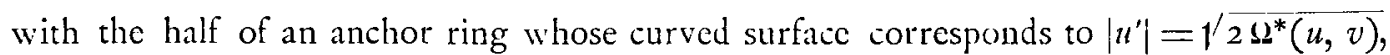
and whose bounding meridian plane corresponds to $v=0$. The two states of motion merge along the curved surface.

This region may be deformed into the region of space between two concentric hemispheres so that the spherival surfaces correspond to the two sections of the anchor ring by the meridian plane, and the plane boundary corresponds to the curved bounding surface of the anchor ring. To each point of this hemispherical solid corresponds two states of motion, which merge along the bounding plane.

Hence, if we consider this solid as doubly taken, and make a reflection of one of the solids about this plane leaving the points of the plane invariant, we obtain a representation of the states of motion in the region between two concentric spheres, which is one-to-one and continuous save along the two bounding spherical surfaces where it is two-to-one. But these two surfaces are each of the same nature as the similar spherical surface in Case I. Thus we reach the following conclusion:

In Case III, when the motion takes place in lbe interior of an oval of zero velocity containing $S$ and $J$, the states of motion are in one-to-one contintuls correspondence with the points between two concentric spberes on each of which opposite points are taken as identical.

The original manifold $u, v, u^{\prime}, v^{\prime}$ represents each state of motion twice. We may unite two solids each bounded by a pair of concentric spheres in such wise that they form a like solid and give a one-to-one continuous representation of the $u, v, u^{\prime}, v^{\prime}$ manifold. Similarly placed points on the two bounding spherical surfaces are to be taken as identical. A complete representation of the motion in ordinary space is obtained by joining an infinite number of such solids so as to fill all of space except the origin. An infinite set of points will, however, correspond to the same state of motion in this representation.

With the aid of entirely similar methods the remaining cases can be discussed.

In Case $I V$, when the motion takes place outside of an oval containing neither $S$ or $J$, the states of motion are in one-to-one continuous correspondence with the points between two conientric spberes on each of which opposite points are taken as identical, provided that tiw oppositely placed non-intersecting closed lines on one of the spheres are excluded. The neighborbood of these lines corresponds to the neigbborbood of infinity in the $x, y$-plane.

The treatment of Case IV is of course based on the nature of the region of motion in the $u, v$-plane. This region is a ring from which two points $w=-\frac{1}{2}+\mu$, $w=\infty$ are to be excluded. The treatment can be made essentially as in Case III.

In Case $V$, when the motion takes place outside of two symmetric ovals containing neither $S$ nor $J$, the states of motion are in two-to-one continuous correspondence with the points of a right circular cylinder from which two smaller circular cylinders with parallel. axes bave been removed. The two states of motion merge along all the boundaries save the lateral boundary of the given cylinder. Along this boundary two points representing 
states of motion from the two different sets are taken as identical if they lie along generators symmetric with respect to a fixed plane through the axis, and are at equal distances from opposite bases. The neighborbood of two symmetric singular generators corresponds to the neighborbood of infinity in the $x, y$-plane.

The fairly complicated geometrical representation in Case. $\mathrm{V}$ is obtained by considering the states of motion $v \supseteq 0$. If $u, v, u^{\prime}$ are taken as rectangular coördinates of a point in space, we obtain at once the representation given.

The stated correspondence in pairs of the states of motion $v=0$, which yield the generators of the given right circular cylinder, is easily verified under the following conventions. To each generator corresponds a single $u$ (inclusive of the singular generators $\left.u=-\frac{1}{2}+u, u=\infty\right)$. Let $\%$ denote the angle between the $u$-axis and the direction of motion at $(u, o)$ in the $u, v$-plane. The ends of the doubly taken generator $\left(v^{\prime}=0\right)$ correspond to $\chi=0, \chi=\pi$ and to $\chi=\pi, \chi=2 \pi$.

Now the values of $u$ for $v=0$ are paired by (23), in such wise that as one point $(u, o)$ goes from $S$ to $J$ along the segment $S J$ of the $u$-axis, the other traverses the two outer segments $S \infty$, and $\infty J$; the two points $(u, 0)$ coincide at $S$ and $J$. By a suitable deformation corresponding paired generators may be placed symmetrically about a plane through the two generators $u=-\mathrm{I}+\mu$ and $u=\mu$.

Paired generators yield the same point in the $x, y$-plane. In order that the values of $\psi$ for paired values of $(u, 0)$ give the same state of motion, these values must differ by $\pi$. This conclusion results at once from the fact that the transformation from one value of $w$ to the other is analytic, and the further fact that associated points are arranged in opposite order on the line $v=o$ in the $u, v$-plane.

Hence the correspondence along the generators is essentially as stated.

In Case VI, when the motion is unrestricted, the states of motion are in one-to-one continuous correspondence with the points of an anchor ring on which points symmetric with respect to the center are taken as identical. The neighborbood of one of the two singular closed lines forming the intersection of the anchor ring and the central plane corresponds to the neigbborbood of infinity in the $x, y$-plane.

In Case VI, as in all the other cases, we consider the states of motion as represented by coördinates $u, v, u^{\prime}$ for which we have $v \gg 0$. This yields two states of motion for a given $u, v, u^{\prime}$, merging for $\left|u^{\prime}\right|=1^{\prime} / \overline{\Omega^{*}(u, v)}$.

Since $u$ and $v$ are unrestricted except for the condition $v \gg 0$ we obtain a twoto-one representation by a right circular cylinder as in Case $V$, except that here no part of the cylinder is removed. Moreover the correspondence along the generators is the same are in Case V.

The representation by a double cylinder so obtained is clearly equivalent to the representation stated.

The results above show that the restricted problem of three bodies presents fundamentally different aspects for different values of the constant of JACOBI. 
There are other problems of analysis situs, such as a partial classification of the possible types of periodic orbits, which can be based on the above results. But the conclusions to be obtained are not sufficiently definite for our purposes.

In the remainder of the present paper I shall confine attention to Case I. Of the other cases, only Case II appears not to present difficulties beyond those of Case I.

PART III.

INVESTIGATION OF POINCARE'S RING 'TRANSFORMATION.

\section{$\$ 9$. \\ Representation of the States of Motion for $\mu=0$.}

The greater simplicity in Case I is closely associated with the fact that here we have an approximation to the two body problem, at least for $u$ sufficiently small or for $C$ sufficiently large. Let us therefore commence with a consideration of the extremely instructive limiting case $\mu=o^{\mathrm{r} 3}$ ).

The body $J$ is of mass I and lies at the origin in the $x$, y-plane while the body $S$ is of mass zero and lies at $(-\mathrm{I}, \mathrm{O})$.

The differential equations and the integral of JacoBs take the form

$$
\begin{gathered}
\frac{d^{2} x}{d t^{2}}-2 \frac{d y}{d t}=x\left(1-\frac{\mathrm{I}}{r^{3}}\right), \quad \frac{d^{2} y}{d t^{2}}+2 \frac{d x}{d t}=y\left(\mathrm{I}-\frac{\mathrm{I}}{r^{3}}\right), \\
v^{2}=U(r) \equiv r^{2}+\frac{2}{r}-C
\end{gathered}
$$

where we have written $r=1^{\prime} \overline{x^{2}+y^{2}}$, and where $v$ denotes the relative velocity of $P$ in the rotating $x, y$-plane. These equations are directly integrable. In fact the particle will move attracted to the fixed boly $J$ of mass unity according to the Newtonian law, and its relative motion in the $x, y$-plane will be in a conic section with focus at $J$ which appears to rotate in a negative sense with unit angular velocity.

If we restrict attention to the case $C>3$ there will be two circles of radii $r^{\prime}<\mathrm{I}$ and $r^{\prime \prime}>$ I along which the relative velocity will be zero. The particle may move within the smaller circle or without the larger circle. These facts come at once from the equation (32). We will confine ourselves to the first case, in which the particle

${ }^{13}$ ) Much of the material presented in this and the following paragraph is given in a different form by PoINCARÉ : see ${ }^{2}$ ). 
necessarily moves in a rotating ellipse, whose semi-major axis and eccentricity we will denote by $a$ and $e$ respectively.

If $\theta$ measures the angle between the radius vector from the $J$ to $P$ and the positive direction of the $x$-axis, the same angular coördinate relative to a fixed line through $J$ is $\theta+t$. Thus, by the law of areas for elliptic motion, we get

$$
r^{2} \frac{d}{d t}(\theta+t)= \pm \sqrt{a\left(\mathrm{I}-e^{2}\right)}
$$

since the mass of $J$ is unity. The positive or negative sign is to be taken according as the motion is direct or retrograde with respect to fixed axes. At an apsidal passage we have $d r / d t=0$. Hence the relative velocity $v$ in the $x, y$-plane is $r d \theta / d t$. If now we note that at a lower apsis we have $r=a(\mathrm{I}-e)$, the above equation yields at once

$$
v= \pm \sqrt{\frac{\mathrm{I}+e}{a(\mathrm{I}-e)}}-a(\mathrm{I}-e)
$$

Substituting this value in $(32)$ and simplifying, we find

$$
b=\frac{-1}{2 \sqrt{a}}+\frac{C}{2} \sqrt{a}
$$

The quantity $b$ here introduced designates the semi-minor axis taken positively or negatively according as the motion with reference to fixed axes is direct or retrograde.

The relation (35), which imposes a single condition upon the elliptic motion, yields us all possible types of motion for the given value of $C$, and no others. In fact by retracing steps it may readily be shown that if (35) obtains, the relation (32) holds true at lower apsidal passage for the given $C$ and thus holds always for the same $C$.

The function $V(a)$ forming the right-hand member of $(35)$ is negatively infinite for $a=0$ and is zero for $a=\mathrm{I}, C$. Its first derivative as to $a$ is constantly positive and its second derivative is constantly negative. Furthermore for $a=\mathrm{I}$ we find $b=\frac{1}{2}(C-\mathrm{I})>\mathbf{I}$. Hence the curve $b=V(a)$, where $a$ and $b$ are taken as rectang-

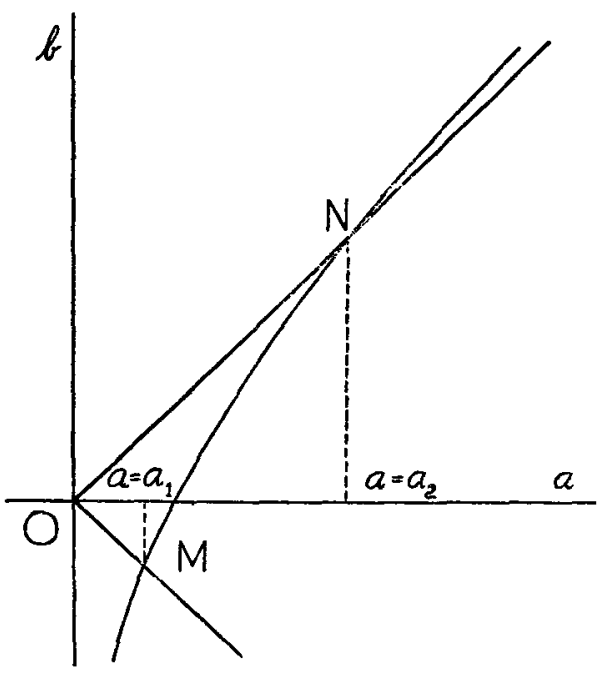

(Fig. 2). 
ular coördinates of a point in the plane (fig. 2), has as asymptote the negative $b$-axis. As $a$ increases, $b$ increases. The curve is constantly concave toward the $a$-axis; it crosses the line $b=-a$ once, and the line $b=a$ twice, once for $a<\mathrm{I}$ and once for $a>\mathrm{I}$. We will denote the values of $a<\mathrm{I}$ for which $b=-a$ and $b=+a$ by $a_{1}$ and $a_{2}$ respectively.

The part of the curve $a_{1} \leq a \leq a_{2}$ for which $|b| \leqslant a$ comprises all points corresponding to a possible elliptic motion which lies within the oval $r=r^{\prime}$, and no others. In fact, if $a<\mathrm{I}$ such a motion cannot lie wholly outside of the oval $r=r^{\prime \prime}$; and if $a>I$ it cannot lie wholly inside of the oval $r=r^{\prime}$.

It is therefore obvious that the totality of orbits to be taken into account corresponds to the range $a_{1} \leq a \leq a_{2}$ giving a segment $M N$ of the curve, where $M$ and $N$ are the first points of intersection of the curve with $b=-a$ and $b=+a$ respectively.

A circular motion is characterized by the condition $b= \pm a$ and we will have the + sign if the motion is direct with respect to fixed axes, and the - sign if it is retrograde. Since the particle $P$ lies nearer to $J$ than $S$ does $\left(r \leq r^{\prime}<\mathrm{I}\right)$ the mean angular velocity of $P$ with respect to fixed axes will exceed that of $S$. Consequently according as we have the + or - sign the motion is direct or retrograde with respect to moving axes also.

The form of the curve $b=V(a)$ shows that we have two and only two possible circular motions, one retrograde of radius $a_{1}$ and the other direct of radius $a_{2}$. The first corresponds to the minimum value of $a$ and the second to the maximum value of $a$.

The fact that the segment $M N$ may be described continuously by a point passing from $M$ to $N$ indicates that the possible types of motion may be arranged in a continuously varying series beginning with the direct circular and ending with the retrograde circular motion. The value $\mathrm{I} / C$ of $a$ corresponds to an orbit of ejection which is thought of as continued analytically after the collision.

From (35) we find at once

$$
\left\{\begin{aligned}
-a_{1} & =-\frac{\mathrm{I}}{2 \sqrt{a_{1}}}+\frac{C}{2} \sqrt{a_{1}} \\
a_{2} & =-\frac{\mathrm{I}}{2 \sqrt{a_{2}}}+\frac{C}{2} \sqrt{a_{2}} .
\end{aligned}\right.
$$

Also if $\alpha$ stands for either $a_{1}$ or $a_{2}$ we find that

and hence that

$$
C= \pm 2 \sqrt{\alpha}+\frac{1}{\alpha}
$$

$$
\frac{d C}{d \alpha}= \pm \frac{\mathrm{I}}{\sqrt{\alpha}}-\frac{\mathrm{I}}{\alpha^{2}} .
$$

This quantity is negative for $\alpha<\mathrm{I}$. Thus we can state the following conclusion, of service to us later : 
As $C$ decreases from $\infty$ to 3 the radii $a_{1}$ and $a_{2}\left(a_{1}<a_{3}<\mathrm{r}\right)$ of the retrograde and direct circular orbits increase from o to $\frac{1}{4}$ and $\mathrm{I}$ respectively.

We shall now inquire into the structure of the three-dimensional manifold of states of motion, which, by the results of $\ 8$, is equivalent to a sphere on which opposite points are taken as identical.

A satisfactory set of variables in this connection is furnished by the semi-major axis $a$ of the rotating ellipse, the longitude $\theta$ of the line of apsides of the ellipse with reference to the $x$-axis, and the mean anomaly $\psi$ of the particle reckoned in the same sense as the motion.

The variable $a$ ranges from $a_{1}$ to $a_{2}$ determining a unique $b$, and thus the figure of the ellipse and the sense of the motion. The variable $\theta$ determines the instantaneous position of the moving ellipse in the $x, y$-plane. The variable $\psi$ determines the instantaneous position of the particle in its ellipse.

Evidently any $a, \theta, \psi$ determines a unique state of motion so long as $a$ is not equal to one of its extreme values $a_{1}, a_{2}$. In this case $\theta$ and $\psi$ become indeterminate. Conversely, any state of motion not corresponding to one of the circular orbits yields a unique $a, \theta, \downarrow$, where it is understood of course that $\theta$ and $\psi$ are angular variables of period $2 \pi$. With these coördinates collision does not present any singularity.

Let us exclude for the moment the states of motion along the circular orbits, and take $a, \theta, \psi$ as cylindrical coördinates of a point in space. The totality of states of motion is then represented in a one-to one and continuous way upon the interior of the hollow cylinder $a_{1}<a<a_{2}$, o $\leqslant \psi \leqslant 2 \pi$. Opposite points on the two bases $\psi=0$ and $\psi=2 \pi$ represent the same state of motion. This solid is therefore of the same nature as an anchor ring.

As $t$ changes, the variables $a, \theta, \psi$ change in accordance with the equations

$$
a=a_{0}, \quad \theta=-t+\theta_{0}, \quad \psi=a_{0}^{-\frac{3}{2}} t+\psi_{0}
$$

in which $a_{0}, \theta_{0}, \psi_{0}$ stand for the values of $a, \theta, \psi$ at $t=0$. These relations are obvious from the definitions of $a, \theta, \psi$ and from the fact that the mean angular velocity $n$ of $P$ fulfills the relation $n^{2} a^{3}=\mathrm{I}$, the mass of $J$ being unity.

Hence we see that the curves representative of motion in $a, \theta, \psi$-space are the spirals on the cylinders $a=$ constant which have pitch $-a^{-\frac{3}{2}}$.

Along the boundary of the cylinders the representation fails. To make plain what is happening here, we observe that a given state of motion on the direct circular motion determines $\theta+\psi$ although it determines neither $\theta$ nor $\psi$. The spirals $\theta+\psi=$ constant on the outer bounding cylinder correspond accordingly to the individual states of motion along the direct circular orbit. Likewise the spirals $\theta-\psi=$ constant on the inner cylinder correspond to the individual states of motion along the retrograde circular orbit. 


$$
\text { S IO. }
$$

\section{The Ring Transformation for $\mu=0$.}

In the special case $\mu=0$, and also in the general case $\mu \neq 0$, the restricted problem of three bodies admits of a species of reduction, observed by PoIncaré ${ }^{2}$ ), by which the representation as a steady flow in three dimensions is replaced by a representation depending on a discrete transformation of a two dimensional ring into itself. We will treat the extremely instructive are $\mu=0$ in the present paragraph.

Let $K$ be any point of the ring $\downarrow=0$ in the representing cylinder $(\$ 9)$, and let $L$ be the point in which the curve of motion through $K$ first meets the ring $\psi=2 \pi$. Corresponding points of these two congruent rings represent the same state of motion. Thus the transformation which talies $K$ to $L$ defines a transformation $T$ of the ring $\psi=0$ into itself,

$$
a^{\prime}=a, \quad \theta^{\prime}=\theta-2 \pi a^{\frac{3}{2}},
$$

where $a, \theta$, o are the coördinates of $K$, and $a^{\prime}, \theta^{\prime}, 2 \pi$ are the coördinates of $L$ [see (37)].

To the essential properties of this transformation (which is a rotation) there correspond properties of the curve of motion, and vice versa. For instance, if the motion is periodic, the point $K$ is taken into itself by a certain number $k$ of repetitions of $T$. If at the same time $\theta$ diminishes by $2 / \pi$, we find, as the necessary and sufficient condition for this type of periodic motion,

$$
\theta^{(k)}=\theta-2 \pi k a^{\frac{3}{2}}=\theta-2 l \pi \text {. }
$$

Here we let $\theta^{(k)}$ denote the value of $\theta$ after $k$ repetitions of $T$. We thus obtain $k / l=a^{-\frac{3}{2}}$ as the condition upon $a$ which makes the motion periodic. Inasmuch as $a$ varies between $a_{1}$ and $a_{2}$, any $k / l$ is possible for which

$$
a_{2}^{-\frac{3}{2}}<k / l<a_{1}^{-\frac{3}{2}} \text {. }
$$

The detaild treatment of the periodic orbits in this case offers no difficulty. We content ourselves with noting two salient facts. The quantity $k / l$ is the mean angular velocity of the particle in fixed space referred to the angular velocity of the moving axes as unit; $k$ circuits of the apparently rotating ellipse are made while that ellipse undergoes $l$ complete revolutions. After a single circuit of the ellipse the line of apsides has regressed through an angle $2 / \pi / k$.

The transformation $T$ admits of a simple geometrical interpretation in the $x$, $y$-plane. To obtain this interpretation we first observe that the condition $\psi=0$ corresponds to lower apsidal passage. Now at apsidal passage the direction of motion in fixed space is at right angles to the radius vector to the origin. The rotation of the $x, y$-plane is also at right angles to this radius vector. Hence the direction of relative 
motion is at right angles to the radius vector in the $x, y$-plane at apsidal passage (the case of zero velocity is included). The converse is also seen to hold.

To distinguish between higher and lower apsidal passage we note that, for $r>a_{2}$, motion at right angles to the radius vector in the $x, y$-plane will necessarily correspond to higher passage inasmuch as the distance from the origin exceeds the maximum semimajor axis. Similarly, for $r<a_{1}$, motion at right angles to the radius vector must correspond to lower passage.

Suppose now that we consider the continuous series of states of motion $y=0$, $d x / d t=0$ in the $x, y$-plane. Here we let $x$ increase from $a_{2}$ to $r^{\prime}$ with $d y / d t>0$, then decrease from $r^{\prime}$ to zero with $d y / d t<0$, and finally increase from zero to $a_{2}$ with $d y / d t>0$. It is clear that the series of states of motion thus obtained is represented by a continuous line in the $u, v, u^{\prime}, v^{\prime}$-space of $\$ 6$ or any equivalent space. For, at $x=r^{\prime}$ we have $d y / d t=0$ as well as $d x / d t=0$, while at $x=0$ we have $\theta=0$, $\downarrow=0$; hence the apparently distinct series merge at the extreme limits for $x$.

As the orbit passes through this series of forms, the states of motion at first corresponds to a higher apsis. This will not change to a lower apsis until the state of motion belongs to one of the two circular motions. This only happens after $a$ has decreased from $r^{\prime}$ to $a_{1}$. This part of the series corresponds to higher apsidal passage.

Likewise, if we commence with the other end of the series, we are led to the conclusion that as $x$ diminishes from $a_{1}$ to zero and increases to $a_{2}$ we have lower apsidal passage.

In other words, if we imagine two series of auxiliary circles drawn with $J$ as center in the $x, y$-plane, the first of retrograde sense and of radius less than $a_{\mathrm{r}}$, the second of direct sense and of radius less than $a_{2}$, the states of motion corresponding to lower apsidal passage $\psi=0$ may be characterized as those for which the path becomes positively tangent to a member of this double series of circles.

It is necessary to make the convention that the null circle $r=0$ is a member of both families and is tangent to any orbit of collision through it in the direction perpendicular to the axis of the cusp at collision. To such a direction corresponds an ideal point of the null circle.

When this convention is made, a complete representation of the states of motion $\psi=0$ will be given by the points of this double series of circles which is obviously of the nature of a ring. The ring may be regarded as given by a species of surface of two leaves in the $x, y$-plane. One of these leaves extends to the circle $a=a_{1}$, the other to the circle $a=a_{2}$; and the two leaves are joined together at the origin along a single infinitude of ideal points as specified above.

Accordingly this ring is in one-to-one continuous correspondence with the ring $\psi=0$ in the $a, \theta, \psi$ space. The explicit nature of the correspondence is obvious also; for, the lines $\theta=$ constant correspond to the radial lines making an angle $\theta$ with the $x$-axis. If $a$ varies from $a_{1}$ to $a_{2}$ while $\theta$ is fixed, the point in the $x, y$-plane moves from the circle of radius $a_{5}$ to the origin and out to the circle of radius $a_{2}$ along the appropriate radial line; the distance from the origin for a given $a$ is $a-\sqrt{a^{2}-b^{2}}$. 
Now let $K$ be a point of the ring, represented in the $x, y$-plane, and consider the orbit in that plane positively tangent to an auxiliary circle through the point $(\psi=0)$. The associated point $L$ into which $K$ goes by the transformation $T$ is then the first subsequent point of positive tangency of the same orbit with one of these circles $(\psi=2 \pi$ ). The specific form (38) of the transformation shows that the successive points of positive tangency lie on one fand the same circle $r=$ constant, and are displaced through equal negative angles about the origin.

Thus our results may be given in the following form:

For $\mu=0$ and $C>3$ there exists a retrograde circular orbit of radius $a_{1}$ and $a$ direct circular orbit of radius $a_{2}\left(a_{1}<a_{2}<\mathrm{I}\right)$ about $J$ as center in the $x$, y-plane. If we construct a family of concentric retrograde circles of radius less than $a_{1}$ and a similar series of direat circles of radius less than $a_{2}$, these circles may be regarded as forming a ring of two leaves joined together at the origin in the $x$, $y$-plane, each leaf being given by one family of circles. For any point $K$ of a circle of the ring a'e bave a positively tangent orbit wbich will again become positively tangent at a point $L$ for the first time. In this way a one-to-one continuous transformation of the ring into itself, taking any point $K$ into the corresponding point $L$, is obtained which leaves radial distances from $J$ unchanged and regresses each point by a central angle $2 \pi a^{\frac{3}{2}}$ about $J$, where $a$ is the semi-major axis of the ellipse of motion of the particle.

Let us visualize these results in the representing sphere of states of motion $(\$ 8)$ by associating with each point of the ring the point of the sphere which represents the state of motion of the positively tangent orbit at the point.

To the direct and circular orbits correspond two stream lines traversing the sphere and joining opposite points of its surface. In fact, in the $u$, $v$-plane $(\$ 5)$ this orbit makes a complete cycle of $J$ before re-entering. During half of this cycle we have $v \gg 0$ and during the other half we have $v \leqslant 0$. In the representing sphere of $\$ 7$, therefore, the beginning and end of the first half cycle (which corresponds to a complete cycle in the $x, y$-plane) will not be represented by a closed line in the ordinary sense but must be represented as stated.

The intermediate circles correspond to a family of similar lines varying continuously from the first to the second line and forming a ring.

No stream line is anywhere tangent to the ring. For, employing the coördinates $x, y, p(\mathbb{I})$ for the orbit and for the intermediate circles, we conclude that the equation of the ring is

$$
x \cos \varphi+y \sin \varphi=0 .
$$

These coördinates fail for $x=y=0$ and we exclude this case for the moment. If $d x, d y, d p$ are the differentials of $x, y, \varphi$ along the orbit, the condition for tangency is

$$
\cos \varphi d x+\sin \varphi d y-x \sin \varphi d \varphi+y \cos \varphi d \varphi=0 \text {. }
$$

But, if we let $s$ stand for the variable arc along the orbit and recall that the orbit 
is positively tangent to the circle, we get from the preceding equation,

$$
\frac{d \varphi}{d s}=\frac{1}{x \sin \varphi-y \cos \varphi}=\frac{ \pm \mathrm{I}}{r} .
$$

That is, the curvature of the orbit and circle coincide. This is impossible, for from this fact alone it would follow that the auxiliary circle itself represents a circular orbit for the given $C$.

If we employ the regularizing transformation of Levi-Civita $(\$ 4)$ in order to consider the case $x=y=0$, the family of auxiliary circles with $I$ as center is carried over into itself, while the orbits near $J$ become approximately rectilinear. The curvature of the small auxiliary circles is large. Hence every orbit passing near $J$ is positively tangent to an auxiliary circle once and only once. The stream lines must all cut the ring in the same sense for $x$ and $y$ small.

The points which at $t=0$ lie on the ring form a surface, which will traverse the whole of the sphere as $t$ increases and each point moves along its stream line. In this way, when $t$ has increased by a suitable amount, any point of the ring will be taken into another. The transformation of the ring so obtained is clearly the same as that given above.

When account is taken of the fact that the circular periodic orbits vary analytically with $\mu$, considerations of continuity render the existence of a similar ring for $\mu \neq 0$ highly probable. To preserve the essentials of the above construction it is only necessary to vary the ring in the representing space with $\mu$. so that it remains bounded by the two varied periodic orbits for the given $C$, and does not become tangent to any of the stream lines. It is apparent that if such a ring is given it may be subjected to deformation.

These considerations of continuity guided PoINCARÉ to a large extent in his analytic construction of a similar ring.

It may be stated in passing that an infinitude of essentially different rings can be formed. One of these, which has a particular importance, is introduced later $(\$ 19)$.

SII.

\section{Analytic Continuation of the Circular Orbits.}

We shall establish that the simple and entirely explicit construction for the ring in the plane of the motion which has been obtained above in the case $\mu=0$ admits of complete extension to the case $\mu \neq 0$.

The circles concentric with the origin in the $x, y$-plane which have been employed in this construction have also appeared as the totality of circular orbits for all greater values of $C(\$ 9)$. In generalizing the ring construction we shall make use of some properties of the analytic continuation of these orbits developed in the present and following paragraph. 
We shall first demonstrate the existence of such a continuation by a method of more geometrical cast than a similar method dereloped by F. R. Moulton ${ }^{14}$ ).

Consider the orbits belonging to the given value of $C$ which cross the $x$-axis perpendicularly in the direct sense $(d y / d t>0)$ at $t=0$, with abscissa nearly equal to $a_{2}$. Let the abscissa of crossing be lenoted by $\bar{x}$. The orbit will coincide with the circular orbit for $\bar{x}=a_{2}$ and $\mu=0$; in this case the first following crossing will lie at $x=-a_{2}$ and will be at right angles to the $x$-axis. If $\psi(\bar{x})$ denotes the value of $d x / d t$ at this second crossing it is clear that $\downarrow(\bar{x})$ is analytic in $\bar{x}$ and $\mu$. This is an immediate corollary of the fact that, when two analytic curves intcrsecting at an angle different from zero depend analytically upon parameters, the coördinates of the point of intersection, the slopes of the curves at the same point, etc. vary analytically with these parameters. The parameter along the curves is assumed to be regular.

Hence we may write $\psi(\vec{x})$ as a power series in $\bar{x}-a_{2}$ and $\mu$, without constant term :

$$
\left.\psi(\bar{x})=\alpha_{2} \bar{x}-a_{2}\right)+\beta_{2} \mu+\gamma_{2}\left(\bar{x}-a_{2}\right)^{2}+\cdots .
$$

If $\alpha_{2}$ is not zero, that is if $d \psi / d \bar{x} \neq 0$ for $\mu=0$, the curve $z=\psi(\bar{x})$ crosses the $\bar{x}$-axis in the $\bar{x}$, z-plane at an angle different from zero for $\mu=0$. In this event it is apparent that the abscissa of the point where the curve $z=\dot{\bar{x}}$ cuts the $\bar{x}$-axis varies analytically from $-a_{2}$ as $\mu$ varies from zero. But the equation of this abscissa is $\psi(\bar{x})=0$. This condition insures precisely that the orbit which intersects the $x$-axis perpendicularly at $x=a_{2}$ in the $x, y$-plane, intersects perpendicularly at the first following crossing. In this way we obtain the analytic and only continuation of the upper half of the circular orbit into another which intersects the $x$-axis perpendicularly twice in succession.

The differential equations (I) and the integral (3) are unchanged if $x, y, t$ are replaced by $x,-y,-t$ respectively. Therefore one concludes that this continuation together with its reflection in the $x$-axis constitutes a complete symmetric periodic orbit, the analytical continuation of the direct circular orbit.

It remains only to consider the condition $\alpha_{2} \neq 0$ which enters essentially into the above reasoning. To this end we consider the function $\psi(\bar{x})$ for $\mu=0$, which by definition denotes the component of the velocity in the direction of the positive $x$-axis in the rotating $x, y$-plane at the second crossing. Inasmuch as a change in the velocity of rotation about the origin at $J$ does not affect this component, $\psi(\bar{x})$ may

14) F. R. Moulton, A Class of Periodic Solutions of the Problem of Three Bodies with Application to the Lunar Theory [Transactions of the American Mathematical Society, vol. VII (1906), pp. 537-577]. The possibility of continuation was first established by H. Porncare, Les methodes nouvelles de la Mécanique Celeste (Paris, Gauthier-Villars, 1892), vol. I, pp. 79-119, and somewhat later in a paper by T. Levi-Civita, Sopra alcuni criteri di instabilità [Annali di Matematica, ser. III, vol. V (I9oo), pp. 221-307], in particular pp. 282-289. 
be interpreted as the radial velocity toward $J i_{n}$ the fixed plane at the time $t_{1}$ of the second crossing.

The particle $P$ in the fixed plane moves in an ellipse with focus at $J$, whose principal axis at the time of first crossing coincides with the $x$-axis since the particle crosses in both the fixed and moving plane at right angles. Let $w$ denote the angle between the radius vector from $J$ to the moving particle and the principal axis of the ellipse, taken in the same sense as the $x$-axis. Let $e$ denote its eccentricity and $a$ its semi-major axis. The equation of the ellipse is then

$$
r=\frac{a\left(\mathrm{I}-e^{2}\right)}{\mathrm{I} \pm e \cos w}
$$

where the + or - sign is required according as the first crossing corresponds to lower or higher apsis. From this equation and the above interpretation of $\psi(x)$ we get

$$
\psi(\bar{x})=\left.\frac{d r}{d t}\right|_{t=t_{\mathbf{t}}}=\left.\frac{\mp a e\left(\mathrm{I}-e^{2}\right) \sin w d w}{(\mathrm{I} \pm e \cos w)^{2}} d \bar{t}\right|_{t=t_{\mathrm{t}}} .
$$

We wish to obtain $d \psi(\bar{x}) / d \bar{x}$ for $\bar{x}=a_{2}$. In this case $\psi(\bar{x})$ vanishes, while $a$ and $b$ reduce to $a_{2}$ for $\bar{x}=a_{2}$. Moreover we have for any $\bar{x}$

$$
a \mp \sqrt{a^{2}-b^{2}}=\bar{x} \text {, }
$$

since the first crossing is at an apsis. If we write

$$
a=a_{2}+\Delta a, \quad b=a_{2}+\Delta b, \quad \bar{x}=a_{2}+\Delta \bar{x}
$$

this relation becomes

$$
\Delta a \mp \sqrt{(\Delta a-\Delta b)\left(2 a_{2}+\Delta a+\bar{\Delta} b\right)}=\Delta \bar{x} .
$$

But $d b / d a$ is positive and greater than I at $a=a_{2}$ while $\lrcorner a$ is of course negative (see fig. 2). The term $\Delta a-\Delta b$ is therefore an infinitesimal of the first order in $\Delta a$. It follows from the proceding equation that $\Delta a, \Delta b$ and $\Delta a-\Delta b$ are of the second order in $\Delta \bar{x}$, and that

$$
\lim _{\bar{x}=a_{2}} \frac{\mp \sqrt{a^{2}-b^{2}}}{\sqrt{x}}=\lim _{\bar{x}=a_{2}} \frac{\mp a e}{\Delta \bar{x}}=\mathbf{I} \text {. }
$$

Dividing through the equation for $\downarrow \overline{(x)}$ by $\lrcorner \bar{x}$ and utilizing the fact just deduced we find

$$
\alpha_{2}=\lim _{\bar{x}=d_{z}} \frac{\psi(\bar{x})}{\sqrt{x}}=\left.a_{2} \sin w \frac{d w}{d t}\right|_{t=t_{1}^{*}} .
$$

Here $t_{1}^{*}$ denotes the value of $t_{1}$ at second crossing in the $x, y$-plane along the direct circular orbit.

Thus the coefficient $\alpha_{2}$ vanishes if and only if the time $t_{1}^{*}$ required for the particle in the circulat orbit to pass from one crossing of the $x$-axis to the next is a multiple of the half period in the fixed plane (i. e. if $w$ is a multiple of $\pi$ ).

If we had commenced with the retrograde circular orbit and had introduced the 
coefficient $\alpha_{1}$, defined precisely as $\alpha_{2}$ was defined, we should have been led to the same condition for the vanishing of $\alpha_{2}$.

Denote by $n$ the mean angular velocity (taken positively) in the direct or retrograde orbit relative to fixed axes. Relative to moving axes the mean angular velocity is then $n \mp \mathrm{r}$, the upper or lower sign to be taken according as we have the direct or retrograde circular motion. The defining equation for $t_{\mathrm{r}}^{*}$ is $(n \mp \mathrm{I}) t_{\mathrm{r}}^{*}=\pi$ and the condition that $\alpha_{2}$ vanishes is that $n t_{1}^{*}=k \pi$. The condition upon $n$ is therefore $n= \pm k /(k-\mathrm{I})$. The lower sign corresponding to retrograde motion yields no possible values of $n$ which is positive by definition.

The upper sign corresponding to direct circular motion gires us $2 / \mathrm{I}, 3 / 2, \ldots$ as the exceptional values of $n$. The relation $n^{2} a_{2}^{3}=I$ together with the second equation (36) determine the corresponding values of $C$. We observe that the greatest value of $n$ yields the least $a_{2}$, and thus the greatest exceptional value of $C(\$ 9)$, namely $\sqrt[3]{32}$.

Our conclusion is that analytical continuation of the retrograde circular orbits is possible for any $C>3$, while analytic continuation of the direct circular orbits is possible for any $C$ not one of these exceptional values.

\12.

\section{Further Properties of the Direct and Retrograde Orbits.}

For our purposes it is essential to go somewhat further into the study of the analytic continuation of the circular orbits. We shall establish the following result:

For $C \supseteq C_{1}>\sqrt[3]{32}$, analytic continuation of the direct and retrograde circular orbits under consideration is possible for $\mu$ sufficiently small and independent of $C$. The distortion of the $x, y$ plane required to take the limiting circular orbits for $\mu=0$ into their analytic continuation for the same $C$ so that corresponding points bave the same mean anomaly may be represented in the form

$$
\left\{\begin{array}{l}
\rho^{\prime}=\rho\left(\sqrt{\mathrm{I}-\mu}+\mu \rho^{2} f(\rho, \theta, \mu)\right), \\
\theta^{\prime}=\theta+\mu \rho^{2} g(\rho, \theta, \mu),
\end{array}\right.
$$

where $\rho^{2}, \theta$ are polar coördinates for $\mu=0$, and $\rho^{\prime 2}, \theta^{\prime}$ are similar coördinates for $\mu \neq 0$, and where $f$ and $g$ are analytic in $\rho, \theta, \mu$ and periodic in $\theta$ of period $2 \pi$ for $\mu$ sufficiently sinall. The quantities $\rho, \rho^{\prime}$ are taken positively or negatively according as $\left(\rho^{2}, \theta\right),\left(\rho^{\prime 2}, \theta^{\prime}\right)$ are points of a direct or retrograde orbit.

Let us begin the proof by noting that the argument of $\$$ II shows that continuation of the circular orbit is possible throughout any restricted range of values of $C \gg C_{1}$ if $\mu$ is sufficiently small. For, $\alpha_{1}$ and $x_{2}$ are analytic functions of $\mu_{0}$ not zero for $C$ in this range, and we may solve the equation $\psi(\bar{x})=0$ for $\bar{x}$ as an analytic function of $\mu$ and $C$, for $\mu$ sufficiently small, in consequence.

In order to demonstrate the first italicized statement it suffices then to establish 
that analytic continuation is possible for all values of $C$ sufficiently large and for $\mu$ sufficiently small but independent of $C$. Our method will be very similar to that employed in $\ \mathrm{I} I$.

To do so we write $\lambda^{2}=\mathrm{I} / C$ and make the change of variables

$$
x-\mu=\lambda^{2} \xi, \quad y=\lambda^{2} n, \quad t=\lambda^{3} \tau
$$

which reduce the equations (I), (3) to the form

where

$$
\begin{gathered}
\frac{d^{2} \xi}{d \tau^{2}}-2 \lambda^{3} \frac{d n}{d \tau}=\bar{\Omega}_{\xi}(\xi, n), \quad \frac{d^{2} n}{d \tau^{2}}+2 \lambda^{3} \frac{d \xi}{d \tau}=\bar{\Omega}_{n}(\xi, n), \\
\left(\frac{d \xi}{d \tau}\right)^{2}+\left(\frac{d n}{d \tau}\right)^{2}=2 \bar{\Omega}(\xi, n)-\mathrm{I},
\end{gathered}
$$

(44) $\bar{\Omega}(\xi, n)=\frac{\lambda^{2}}{2}\left[\mu+2 \mu \cdot \lambda^{2} \zeta+\lambda^{+} \xi^{2}+\lambda^{4} n^{2}\right]+\frac{1-\mu}{\sqrt{\xi^{2}+n^{2}}}+\frac{\mu \lambda^{2}}{\sqrt{\left(1+\lambda^{2} \xi\right)^{2}+\lambda^{4} n^{2}}} \cdot$

To $J$ in the $x, y$-plane corresponds the origin in the $\xi$, n-plane. The transformation effects a translation together with a change in the units of length and time.

The effect of changing $\lambda$ to $-\lambda$ in these equations in the same as changing $\tau$ to $-\tau$. It follows that we may confine our attention to the continuation of the direct circular orbits for $\lambda$ positive or negative. The negative value of $\lambda$ for a given $C$ will then yield retrograde orbits in the $x, y$-plane for positive $\lambda$.

Referring back to the interpretation of the case $\mu=0$ in the $x, y$-plane, we infer that, for $\mu=o$, the motion is in an ellipse

$$
\rho=\frac{\alpha\left(I-\varepsilon^{2}\right)}{I \pm \varepsilon \cos w}
$$

in the fixed plane. Here $x$, expressed in the same units as $\xi, r$, and $\varepsilon$ denote the seni-major axis of the ellipse and its eccentricity respectively; $\rho$ denotes the radius vector from the particle to the origin in the same units, and $w$ is the angle between this radius vector and the instantaneous position of the $\xi$-axis at $\tau=0$. It is obvious that the semi-axes $\alpha$ and $\beta$ of this ellipse in these units are $\lambda^{-2}$ times the corresponding semi-axes $a$ and $b$ in the former units. By means of this fact we obtain the equation

$$
\sqrt{\alpha}-\frac{1}{\sqrt{x}}=2 \lambda^{3} \beta
$$

directly from (35). This is the necessary and sufficient condition that motion is possible in a rotating ellipse of semi-axes $x$ and $\beta$, for the given value of $\lambda$.

In the limiting case $\lambda=0,(C=\infty), \mu=0$ the condition becomes $x=\mathrm{I}$ i. e. the ellipses have a unit semi-major axis but are otherwise unrestricted. There is then a circular orbit of radius unity.

Now consider a series of orbits in the from the $\bar{\xi}$-axis perpendicularly at $\tau=0$ with abscissa $\bar{\xi}$, and let $\chi(\bar{\xi})$ denote the 
$\xi$-component of the velocity at the first following intersection with the $\xi$-axis $\left.{ }^{15}\right)$. If $\mu=\mathrm{o}, \lambda=\mathrm{x}, \bar{\xi}=\mathrm{I}$ we have $\chi(\overline{\bar{\xi}})=\mathrm{o}$ inasmuch as the motion is in the circular orbit of radius I; and in the neighborhood of this set of values $\chi(\bar{\xi})$ is analytic in $\mu, \lambda, \bar{\xi}$ and may be written as a power series in $\mu, \lambda, \bar{\xi}-1$.

For $\lambda=0$ the equations (43) of motion reduce to the equations of motion for a particle at $(\xi, n)$ in a fixed $\check{\zeta}$, n-plane attracted by a body of mass $I-\mu$ at the origin. The series of orbits we are considering will be eliipses in this plane whose focus is at the origin and whose principal axis is the $\%$-axis. We conclude that $\%(\vec{\xi})$ is identically zero for $\lambda=0$.

Now the initial conditions at $t=0$ are

$$
\left.\xi=\bar{\xi}, \quad n=0, \quad \frac{d \xi}{d t}=0, \quad \frac{d n}{d t}=\sqrt{2 \bar{\Omega}(\bar{\xi}}, 0\right)-\mathrm{I} ;
$$

these are analytic in $\bar{\xi}, \mu$ and $\lambda$. Moreover in the differential equations every term which involves $\lambda$ involves $\lambda$ to at least the third power. Therefore if we put $\lambda=0$ in the differential equation alone and not in the initial conditions, the somponent of the velocity at the time of second crossing of the $x$-axis will be affected only in terms of at least the third order in $\lambda$. But this component becomes now identically zcro. Hence $\%(\bar{\xi})$ contains no terms of less than the third order in $\lambda$, and we have necessarily

$$
\chi(\bar{\xi})=\lambda^{3}\left[\alpha_{3}(\bar{\xi}-\mathrm{I})+\beta_{3} \mu+\gamma_{3} \lambda+\delta_{j}(\bar{\xi}-\mathrm{I})^{2}+\cdots\right] .
$$

The necessary and sufficient condition that the second crossing be at right angles is $\chi(\bar{\xi})=0$. If the factor $\lambda^{3}$ be removed, it appears that analytic continuation of the circular orbits into an orbit which cuts twice at right angles will be possible provided that $\alpha_{3}$ is not zero. Also the equations (43) are unaltered if $\xi, n$, $\tau$ be replaced by $\xi,-n,-\tau$ so that orbits for which $\chi(\bar{\xi})=0$ yield symmetric periodic orbits in the $\xi$, $n$ plane.

It is necessary then to evaluate $x_{3}$. To this end we put $\mu=0$ and write $\bar{\xi}=\mathrm{I}+\lambda^{\frac{1}{2}}$ in order that the first term of the series for $\%(\overline{\bar{\xi}})$ becomes the principal term. Thus we obtain

$$
\alpha_{3}=\lim _{\lambda=0} \frac{\chi(\bar{\xi})}{\lambda^{\frac{7}{2}}}, \quad\left(\bar{\xi}=\mathrm{I}+\lambda^{\frac{\mathrm{I}}{2}}, \mu=0\right) .
$$

Since we have $\mu=0$, we find from (45) [compare with (40)]

$$
\chi(\bar{\xi})=\left.\frac{\mp \alpha \varepsilon\left(\mathrm{I}-\varepsilon^{2}\right) \sin w}{(\mathrm{I}-\varepsilon \cos w)^{2}} \frac{d w}{d \tau}\right|_{\tau=\tau_{\mathrm{I}}}
$$

where $\tau_{1}$ is the time of second crossing of the $\xi$-axis. But, as $\lambda$ approaches zero, $\bar{\xi}$

15) It is obvious that $\chi(\bar{\xi})=\lambda \psi\left(\lambda^{-2} \bar{\xi}\right)$ where $\psi$ is the function defined in $S$ II. 
approaches I by (46), and the orbit approaches a circular orbit of radius unity; hence $\varepsilon$ approaches zero, $w$ approaches $\pi$, and $d w / d \tau$ approaches unity.

Also we have by definition of $\bar{\xi}$ [compare with (4I)]

$$
\Delta \alpha \mp \sqrt{(\Delta \alpha-\Delta \beta)(2+\Delta x+\Delta \beta)}=\lambda^{\frac{1}{2}},
$$

where we have written $\alpha=\mathrm{I}+\Delta x, \beta=\mathrm{I}+\Delta \xi, \bar{\xi}=\mathrm{I}+\lambda^{\frac{1}{2}}$. Now the equation (46) shows that $\Delta \alpha$ is of the third order in $\lambda$. Consequently from (49) we infer that the radical $\mp \sqrt{\alpha^{2}}-\overline{\beta^{2}}$ or $\mp x \varepsilon$ has the same principal part as $\lambda^{\frac{1}{2}}$. Combining these results we are able to write

$$
x_{j}=\left.\lim _{\lambda=0} \frac{\sin w}{\lambda^{3}}\right|_{\tau=\tau_{i}} .
$$

The significance of $w$ should be recalled. The particle $P$ for $\mu=0$ and the given $\lambda$ is projected from the point $\xi=I+\lambda^{\frac{1}{2}}, n=0$ of the $\xi$-axis perpendicularly to that axis at $\tau=0$ and crosses it again near to $\zeta=-\mathrm{I}, n=0$. The angle $w$ is the true anomaly relative to fixed axes at the instant of second crossing measured from the position of the $\xi_{\text {-axis }}$ at $\tau=0$.

If $\vee$ be the mean angular velocity of the particle in the fixed plane its mean angular velocity relative to the moving $\xi$, $n$-plane is $\nu-\lambda^{3}$, since the $\xi$, $n$-plane rotates at angular velocity $\lambda^{3}$. Consequently, when the true anomaly is $\pi$ in the fixed plane, the corresponding angle is $\pi\left(\mathrm{r}-\lambda^{3} / v\right)$ in the moving plane, and the difference is $\lambda^{3} / \nu$. Since this difference is of the third order in $\lambda$, and since the difference remains approximately unaltered through a small interval of time, it is apparent that at the time of the second crossing the value of $w$ is $\pi\left(1+\lambda^{3} / v\right)$ to terms of higher order. In consequence the quantity $\sin w$ has the principal part $\lambda^{3} \pi$. Returning now to (50), we find that $\alpha_{3}$ has the value $\pi$.

Hence analytic continuation is possible for $|\lambda| \leq C_{1}^{-\frac{1}{2}}\left(\right.$ i. e. $C \supseteq C_{1}$ ) and $\mu$ sufficiently small. It remains to demonstrate the second part of the italicized statement and this can be done very simply.

According to what has been proved above we may write the equations of the family of periodic orbits in the form

$$
\xi=A(\lambda, \mu, \tau), \quad n=B(\lambda, \mu, \tau)
$$

where $A$ and $B$ are analytic in $\lambda, \mu, \tau$ and periodic in $\tau$ for $|\lambda| \leq C_{1}^{-\frac{1}{2}}$ and $\mu$ sufficiently small. For $\lambda=0$ the equations of motion have been noted to reduce to those obtained when a body of mass $\mathbf{I}-\mu$ at the origin attracts a particle at $(\xi, n)$ in a fixed $\xi$, n-plane, in which case the family of periodic orbits above becomes circular, and the equations of the circular motion are

$$
\xi=(I-\mu) \cos (I-\mu)^{-1} \tau, \quad n=(I-\mu) \sin (I-\mu)^{-1} \tau
$$

by the equations (43). 
The variable $\lambda$ occurs to no lower power than the second in the differential equations and initial conditions. We infer that the above squations may be written $\xi=(I-\mu) \cos (I-\mu)^{-1} \tau+\dot{\lambda}^{2} f_{1}(\dot{\Lambda}, \mu, \tau), \quad n=(I-\mu) \sin (I-\mu)^{-1} \tau+\lambda^{2} g_{1}(\lambda, \mu, \tau)$ where $f_{1}$ and $g_{1}$, are analytic in their arguments and periodic in $\tau$.

For $\lambda=0$ the period $T$ reduces to $2 \pi(\mathrm{I}-\mu)$; the difference $T-2 \pi(\mathrm{I}-\mu)$ is analytic in $\lambda, \mu$, and will contain a factor $\lambda^{2}$. Now let us introduce the mean anomaly

$$
\tau^{*}=\frac{2 \pi \tau}{T}
$$

which differs from $(I-\mu)^{-1} \tau$ by terms of the second order in $\lambda$. When $\tau$ is expressed in terms of $\tau^{*}$ in the equations for $\xi, n$ they retain a similar form:

$$
\xi=(I-\mu) \cos \tau^{*}+\lambda^{2} f_{2}\left(\lambda, \mu, \tau^{*}\right), \quad n=(I-\mu) \sin \tau^{*}+\lambda^{2} g_{2}\left(\lambda, \mu, \tau^{*}\right) \text {. }
$$

These equations may be modified as follows:

$$
\sqrt{\xi^{2}+n^{2}}=1-\mu+\lambda^{2} f_{3}\left(\lambda, \mu, \tau^{*}\right), \quad \arctan \frac{n}{\zeta}=\tau^{*}+\lambda^{2} g_{3}\left(\lambda, \mu, \tau^{*}\right) .
$$

Here $f_{2}, g_{2}, f_{3}, g_{3}$ are analytic in all their arguments and periodic of period $2 \pi$ in $\tau^{*}$.

Let $\rho^{\prime 2}, \theta^{\prime}$ denote polar coördinates in the $x$, $y^{\prime}$-plane with $J$ as origin, so that

$$
\rho^{\prime}=\lambda \sqrt[4]{\xi^{2}+n^{2}}, \quad \theta^{\prime}=\arctan \frac{n}{\xi} .
$$

The above equations may be written

$$
\rho^{\prime 2}=\lambda^{2}\left(\mathrm{I}-\mu+\lambda^{2} f_{3}\left(\lambda, \mu, \tau^{*}\right)\right), \quad \theta^{\prime}=\tau^{*}+\lambda^{2} g_{;}\left(\lambda, \mu, \tau^{*}\right) .
$$

But for $\mu=0$ the values $\rho^{2}, \theta$ of $\rho^{\prime 2}$, $\theta^{\prime}$ reduce to

$$
\rho^{2}=\lambda^{2}\left(I+\lambda^{2} f_{j}\left(\lambda, 0, \tau^{*}\right)\right), \quad \theta=\tau^{*}+\lambda^{2} g_{3}\left(\lambda, o, \tau^{*}\right) \text {. }
$$

Here $\rho$ is really independent of $\tau^{*}$ since the motion is circular; and $\theta$ is equal to $\tau^{*}$, the mean anomaly. Taking note of these facts and solving for $\lambda$, $\tau^{*}$, we obtain

where $f_{4}$ is analytic in $p$.

$$
\lambda=p\left(I+p^{2} f_{4}(p)\right), \quad \tau^{*}=\theta,
$$

Let us associate $(\rho, \theta)$ with $\left(\rho^{\prime}, \theta^{\prime}\right)$ so that these correspond to the same value of $\lambda$ and $\tau^{*}$ (i.e. $C$ is the same for the disturbed and circular orbits, and the mean anomaly is the same at associated points). If we eliminate $\lambda$ and $\tau^{*}$ by the aid of the last equations we get

$$
\rho^{\prime}=\rho\left(\sqrt{I-\mu}+\rho^{2} f_{5}(\rho, \theta, \mu)\right), \quad \theta^{\prime}=\theta+\rho^{2} g_{5}(\rho, \theta, \mu) .
$$

But this reduces to an identity for $\rho^{\prime}=\rho, \theta^{\prime}=\theta$ and $\mu=0$. We conclude that $f_{\text {; }}$ and $g_{5}$ contain a factor $\mu$. This leads us to the final form $(42)$ of equations valid for $C \supseteq C_{1}$ and $\mu$ sufficiently small.

The relation of $\rho, \rho^{\prime}$ to $\lambda$ makes it clear that positive values of $\rho, \rho^{\prime}$ correspond to direct orbits, and that negative values correspond to retrograde orbits. 
As $\mu$ varies from zero we may conceive of the family of retrograde and direct orbits as obtained by suitable distortions of the plane from the family of retrograde and direct circular orbits respectively. This fact is at once apparent from (42), which closely prescribes the nature of the distortions.

$$
\text { \I3. }
$$

The Ring Transformation for $\mu \neq 0$.

For a given value $C_{1}$ of $C$ and for $\mu=0$ we were led in $\$ ro to conceive of the families of direct and retrograde circular orbits for $C \supset C_{2}$ as generators of the two leaves of a ring. The two leaves were joined along corresponding radial lines at the origin.

For $\mu \neq 0$ we shall consider the family of auxiliary curves formed by the analytic continuation of the direct and retrograde orbits for $C \gg C_{1}$ as constituting a similar ring. According to the results obtained in the preceding paragraphs this extension is possible for $C_{1}>\sqrt[3]{3^{2}}$ if $\mu$ be taken sufficiently small.

The parameters defining a point $\left(\rho^{\prime}, \theta^{\prime}\right)$ of the ring $(\oint$ I 2$)$ will be taken to be $\rho$ and $\theta$; here $\rho^{\prime 2}$ and $\theta^{\prime}$ are the polar coordinates of a point with reference to the body $I$, while $\rho^{2}$ is the radius of the undisturbed circular orbit $(\mu=0)$ with the same $C$ as the orbit on which $\left(\rho^{\prime 2}, \theta^{\prime}\right)$ lies, and $\theta$ represents the mean anomaly of the particle $P$ in its orbit measured from the crossing of the $x$-axis. The variables $p$ and $p^{\prime}$ by convention are positive or negative according as $\left(\rho^{\prime 2}, \theta^{\prime}\right)$ belongs to a direct or retrograde orbit. The parameters $\rho, \theta$ will range over the values

$$
-\sqrt{a_{1}} \leq \rho \leq \sqrt{a_{2}}, \quad 0 \leq \theta \leq 2 \pi
$$

where $a_{1}$ and $a_{2}$ denote the radii of the retrograde and direct orbits respectively for the given value $C$, of $C$.

As a first step toward generalizing the transformation of the ring into itself we observe that for sufficiently small every orbit becomes positively tangent to one of the auxiliary curves of the ring within a suitable interval of time $\tau$, where $\tau$ is independent of the orbit.

The statement holds so far as it refers to the case $\mu=0$ since every rotating ellipse becomes positively tangent to one of the circular orbits of the ring at every lower apsidal passage.

If the statement is not true there exist orbits, for $\mu$ arbitrarily small, which do not become positively tangent to the curves of the ring in an arbitrarily long interval $\tau$ of time. Let $L$ be some limiting position of the particle, distinct from $J$, near the beginning of the intervals $\tau$ for a sequence of orbits such that $\lim \varphi=0, \lim \tau=\infty$.

Any liniting orbit of the scquence through $L$ is either a rotating ellipse which becomes tangent to an auxiliary circular orbit at regular intervals, or else it coincides with one of the circular orbits which limit the ring. 
The first case cannot arise. For, if that alternative held, a small segment of the limiting orbit could be selected lying within the ring for $\mu=0$ and cutting across the circles in opposite senses at its two extremities. A nearby segment of one of the series of orbits, for some $\mu \neq 0$ but small, could then be found lying within the analytic continuation of the ring whose extremities cut the auxiliary curves in opposite senses. But this would imply an intermediate point of tangency to one of these curves. Such a point does not exist, by hypothesis.

In making this argument $I$ have purposely ignored the possibility that the limiting orbit is ejectional. To understand the nature of this case it is convenient to recur to the equations of Levi-Civita $(\$ 4)$. The conformal transformation ( 13 ) from the $x$, $y$-plane to the $p, q$-plane which lead to these equations yields transformed orbits analytic near the origin, while the family of auxiliary curves goes over into a family of curves about the origin which are approximately circular. In fact $?^{\prime}=\sqrt[4]{x^{2}+y^{2}}$ is the radius vector while $\theta^{\prime} / 2$ is the vectorial angle in the $p, q$-plane, so that equations (42) yield this fact directly. By the phrase "approximately circular" I shall mean that by a suitable magnification about the origin these direct and the retrograde curves may be transformed into two families of similar curves which go over analytically into the unit circle.

As the orbit in the $p, q$-plane is allowed to vary through the origin the radial lines to the point of tangency of the orbit with the auxiliary periodic orbit clearly comes to a definite limiting position and determines a limiting normal direction. This limiting position corresponds to the polar coördinates $\rho^{\prime}=0, \theta^{\prime}=\frac{1}{2} \theta_{0}$ or $\frac{1}{2} \theta_{0}+\pi$ in the $p, q$-plane. There is a unique corresponding direction in the $x, y$-plane given by the polar coördinates $p^{\prime 2}=0, \theta^{\prime}=\theta_{D}$. The orbit through the origin thus obtained will be defined to be tangent at the $i d e a l$ point $\left(0, \theta_{0}\right)$ to the null auxiliary curve.

With this convention the possibility that the limiting orbit is an orbit of ejection is also to be excluded since any orbit passing near $J$ for 1 small clearly becomes positively tangent to an auxiliary curve.

To exclude the second case we need to make use of a fundamental property of the first equation ( 10 ) of normal displacement which was found in $\$ 3$.

The coefficients in this linear differential equation of the second order in $\delta n$ are analytic in the parameter $\mu$, provided that the orbit of reference varies analytically with $\mu$. If then, for a particular value of $\mu$, say $\mu_{o}$, all of the solutions of the differential equations for $\delta n$ vanish at least once for $t_{\mathrm{o}}<t<t_{1}$, all of the solutions $\delta n$ will continue to vanish in the same interval of time for $\left|\mu-\mu_{0}\right|$ sufficiently small. This conclusion applies in particular to a periodic orbit of reference. Here the coefficients of the equation are necessarily periodic, having the period of the orbit.

Owing to the interpretation of the actual normal displacement of a varied orbit as equal to $\varepsilon \delta n$ ( $\varepsilon$ small) to terms of higher order, we may infer that, if all the solutions of the equation of normal displacement along a periodic orbit vanish for $\mu=\mu_{0}$ within some interval $\tau$, then all slightly varied orbits for $\left|\mu_{-}-\mu_{0}\right|$ small will cut the analytic continuation of the periodic orbit at least once within the same interval $\tau$. 
Hence, if for $\mu=0$ the direct or retrograde circular orbit lead to an equation for normal displacement whose solutions vanish, every orbit for $\mu \neq 0$ lying sufficiently near to the continuation of that orbit will cut that :continuation arbitrarily often. Between three successive points of cutting there is necessarily at least one point of positive tangency to the family of auxiliary periodic orbits, namely a point on the segment of the orbit lying within the ring. Hence the second possibility will also be excluded if the equation of normal displacement for $\delta n$ has the stated property.

The precise meaning to be attached to the phrase " one orbit is near another" is that $x, y, d x / d t, d y / d t$ for the two orbits differ by a small quantity throughout the interval under consideration.

But the equations of normal displacement of the direct and retrograde circular orbits are readily found from the equations (IO), (II) and (I2) to be

$$
\frac{d^{2} \delta n}{d t^{2}}+a_{2}^{3} \delta n=0 \text { and } \frac{d^{2} \delta n}{d t^{2}}+a_{1}^{3} \delta n=0
$$

respectively, whose familiar solutions obviously vanish as required.

To derive the first of these equations we write first the equation of the circular orbit

$$
x(t)=a_{2} \cos \sigma t, \quad y(t)=a_{2} \sin \sigma t .
$$

From these equations we find

$$
f(w)=a_{2} e^{a t t-1}
$$

where $t$ is the function of $w$ defined by (12) i. e. $t=w / a_{2} \sigma$.

Putting $w=s+1=\mathrm{I} n$ we find

and thence

$$
f(w)=a_{2} e^{-\frac{n}{a_{2}}}\left(\cos \frac{s}{a_{2}}+\sqrt{-1} \sin \frac{s}{a_{2}}\right),
$$

$$
F(s, n)=\frac{\mathrm{I}}{2} a_{2}^{2} e^{--\frac{4 n}{a_{2}}}+\frac{\mathrm{I}}{a_{2}} e^{-\frac{n}{a_{2}}}-\frac{C}{2} e^{-\frac{2 n}{a_{2}}} .
$$

Substituting these values for $f$ and $F$ in the first equation (10), and eliminating $C$ by means of the second equation $(36)$, we obtain the equation of normal displacement along the direct circular orbit as given above. The equation of normal displacement along the retrograde orbit is found in precisely the same way.

We have thus ontlined a complete proof that for $\mu$ small any orbit has a point of positive tangency to an auxiliary curve of the ring within some interval $\tau$ of time. Thus there will be an infinite number of such points on each orbit.

For a given $\mu$, taken sufficiently small, no points of positivi tangency on an orbit can coincide or disappear as the orbit undergoes continnous variation. This property holds for $\mu=0$ since successive points of tangency arise at successive lower apsidal positions of the particle.

If $\mu \neq 0$ we proced by first observing that it is not necessary to consider the immediate vicinity of $J$. For, the use of the variables $p, q$ of Levi-Civita $(\$ 4)$ makes 
it geometrically clear that the nearly rectilinear orbit in the $p, q$-plane is positively tangent to one and only one of the small approximately circular direct or retrograde auxiliary curves about $J$, and that with variation of the orbit this point of tangency varies continuously in the ring.

Furthermore, if the curvature of the orbit at the point of positive tangency does not equal that of the auxiliary orbit, the statement holds. For if $p(x, y)$ denotes the angle which the tangent to the auxiliary curve through $(x, y)$ makes with the $x$-axis, the condition of positive tangency is

$$
W(t, \lambda)=\frac{d y}{d t} \cos \varphi-\frac{d x}{d t} \sin \varphi=0 \quad\left(\frac{d x}{d t} \cos \varphi+\frac{d y}{d t} \sin \varphi>0\right),
$$

where $\lambda$ denotes the parameter on which variation of the orbit depends. If $d W(t, \lambda) / d t$ vanishes also, we should have a double point of tangency. Barring this possibility for the moment, we see that $W(t, \lambda)=0$ will have a simple root varying continuously with $\lambda$.

If we let $\varphi_{1}$ denote the angle between the tangent to the orbit and the $x$-axis the condition for a double point of tangency may be written

$$
\frac{d}{d t}\left[v \sin \left(\varphi-\varphi_{1}\right)\right]=0
$$

where $v$ denotes the velocity (not zero in the case that $\mu$ is small), and where $\varphi$ equals $\varphi_{1}$ at the instant of tangency. From this form of condition we obtain $d \varphi / d t=d \varphi_{1} / d t$. But, because of the tangency, $d \varphi_{\mathrm{I}} / d t$ is equal to $d \varphi^{\prime} / d s_{1}$ multiplied by $d s / d t$ where $s_{1}$ and $s$ denote the arc length along the auxiliary curve and the given orbit respectively. 'Thus $d \varphi / d s$ and $d \varphi_{1} / d s_{1}$ are equal, and the condition $d W(t, \lambda) / d t=0$ is precisely the condition for equality of curvatures along the given orbit and the tangent auxiliary curve.

We may deduce an explicit form for this condition. The curvature $d \psi / d s$ at a point of an orbit is

$$
-\frac{2}{\sqrt{2 \Omega(x, y)-C}}+\frac{-\boldsymbol{\Omega}_{x}(x, y) \sin \varphi+\Omega_{y}(x, y) \cos ?}{2 \Omega(x, y)-C}
$$

by equations ( 5 ). If $C^{\prime}$ is the value of the constant of JACoBr along the auxiliary orbit, at a point where the curvature is the same as for the positively tangent orbit with the given $C$, the angle $p$ is the same for both curves, and we obtain readily the desired condition

$$
\left(-\mathbf{\Omega}_{x}(x, y) \sin p+\mathbf{\Omega}_{y}(x, y) \cos \varphi\right)\left(\frac{\mathrm{I}}{\sqrt{2 \Omega(x, y)-C}}+\frac{\mathrm{I}}{\sqrt{2 \Omega(x, y)-C^{\prime}}}\right)=2 .
$$

We shall only use this equation to note that for $C=C^{\prime}$ this condition can only hold if we have $d \varphi / d t=$ - I by ( $s)$. Now for $\mu=0$, and along the circular orbits, $\mathrm{I}+d \varphi / d t$ is the angular velocity relative to a fixed plane and cannot be zero. Therefore for $\mu$ small, and for orbits near the analytic continuations of the circular orbits 
forming the boundaries of the ring equality of curvatures will not be possible; in fact under these conditions $C^{\prime}$ is nearly equal to $C$.

It is easily seen further that near the two boundaries of the ring the difference in the curvatures of the auxiliary curve and the positively tangent orbit is of the same order as the difference $C^{\prime}-C$.

It has now been established that equality of curvatures is not possible near to $J$ or the boundaries of the ring, although the difference in curvatures approaches zero here. Since the curvatures of the auxiliary curves and of the orbits are continuous functions of $x, y, d x / d t, d y / d t$ and $\mu$, except at $J$, and since the curvatures are unequal for $\mu=0$, it follows that the curvatures are unequal throughout the ring for sufficiently small values of $\mu$.

The periodic orbits for different values of $C$ have been computed by HILL and by DARwin. Their results indicate that all the conditions which have been imposed in the present paragraph are fulfilled for a wide range of values of $\mu$ and $C_{\mathrm{r}}$.

Let $\rho, \theta$ be the parametric coördinates of any point $K$ of the ring defined above. The orbit positively tangent to the auxiliary curve at $K$ will again be positively tangent at a first following point $L$ whose parametric coördinates may be denoted by $\bar{\rho}, \bar{\theta}$. In this way a transformation $T$ of the ring into itself, taking each point $K$ to $L$ is obtained; for $\mu=0$ the transformation takes the explicit form [see (38)]

$$
\bar{\rho}=\rho, \quad \bar{\theta}=\theta-2 \pi a^{\frac{3}{2}}
$$

where $\rho, \theta$ are polar coordinates, and where $a$ is the function of $\rho$ defined by the equation $a-\sqrt{a^{2}-b^{2}}=\rho$ taken in conjunction with (35). This yields the approximate form of the transformation for $\mu$ small.

The transformation $T$ thus defined is evidently a precise generalization of the transformation given in $\$$ 10 for the case $\mu=0$.

It remains to give a brief treatment of the transformation $T$ along the boundary of the ring. At a point of positive tangency in the neighborhood of this boundary the orbit under consideration lies near to the continuation of the circular orbits, and the normal displacement (see $\ 4$ ) is approximately represented by $\varepsilon \delta n$, where $\varepsilon$ is a small constant and $\delta n$ is a solution of the differential equation of normal displacement. On the other hand the normal displacement of an auxiliary periodic orbit from the periodic orbit forming the boundary of the ring is approximately of the form $\varphi(t) \delta C$, where $\delta C$ is the difference between the value of the constant of JACOBI for the auxiliary orbit and for the orbit forming a boundary of the ring, and where $\varphi(t)$ is not zero and is periodic. The approximate form of the condition for tangency of the given orbit with an auxiliary curve given by an auxiliary periodic orbit is that $\delta C$ can be found so that

$$
\varepsilon \delta n=\varphi(t) \delta C, \quad \varepsilon \frac{d \delta n}{d t}=\frac{d \varphi(t)}{d t} \delta C
$$

It is understood of course that all terms but those of the first order are omitted in these equations. 
The condition for this is precisely

$$
\delta n \frac{d \varphi(t)}{d t}-\varphi(t) \frac{d \delta n}{d t}=0
$$

Under the restriction that has been obtained upon the curvature of the auxiliary curves, these will have curvature differing from that of a positively tangent orbit near the boundary of the ring by terms of the same order as $\delta C$ above. Thus none of the roots of the above equation can be multiple.

This leads us to the conclusion that, if the orbit of the particle lies near the limiting orbit of the ring and is positively tangent to a curve of the auxiliary family at $t=t_{\mathrm{v}}$, a root of the above equation lies in the vicinity of $t=t_{\mathrm{o}}$. We are also led to the conclusion that the first following point of tangency at $t=t_{\mathrm{t}}$ occurs for $t$ in the vicinity of the succeeding root of the above equation.

Consequently, if we choose $\delta n$ proportional to that solution of the linear differential equation of normal displacement which makes the left-hand member of the above equation ranish at $t=t_{\mathrm{o}}$, and if $t=t_{\mathrm{1}}$ is the first following root of this equation, the point of the boundary of the ring corresponding to $t=t_{\mathrm{o}}$ may be defined as carried into the point corresponding to $t=t_{1}$. By this convention the transformation $T$ becomes one-to-one and continuous over the ring inclusive of the boundary.

Every one-to-one and continuous transformation of a simple closed curve into itself is associated with a uniquely determined number $x$ such that if the transformation be repeated $m$ times $(m=0, \pm \mathrm{I}, \pm 2, \ldots$ ), every point of the curve has made a number of complete circuits lying between the greatest integer less than and the least integer greater than $m x$. This fact is apparent if the transformation is the distortion of a rotation, when $\alpha$ is merely the angular rotation effected by the transformation. We shall term this number $\alpha$, first considered by PoINCARÉ ${ }^{16}$ ), the coefficient of rotation.

The transformation $T$ takes either boundary of the ring into itself. We will let - $\sigma$ and - - stand for the coefficients of rotation along the direct and retrograde boundaries of the ring respectively. For $\mu=0$ we have $\rho=2 \pi a_{1}^{\frac{3}{2}}, \sigma=2 \pi a_{2}^{\frac{3}{2}}$ according to the results of $\$$ Io.

Our main conclusions may now be summarized as follows:

For $\mu$ sufficiently small and for each value of $C_{\supseteq} \geq C_{1}>\sqrt[3]{32}$ there exists a retrograde periodic orbit and a direct periodic orbit about $\vec{J}$ in the $x, y$-plane, analytic continuations of the retrograde and direct circular orbits for $\mu=0$ respectively. These orbits may be regarded as forming a ring of two leaves in the $x, y$-plane joined together at the point $J$, one leaf being given by the family of retrograde periodic orbits for $C \supseteq C_{1}$, and the other by the family of direct periodic orbits for $C \supseteq C_{1}$. For any point $K$ of a periodic

r6) H. PoIncaré, Sur les courbes definies par les équations différentielles ( $3^{\text {me }}$ partie) [Journal de Mathématiques pures et appliquées, $4^{\text {me }}$ série, t. I (1885), pp. 16 $6_{-2}^{-244}$ ]; in particular pp. 220-244. 
orbit of the ring we have a unique positively tangent orbit, for the given value $C_{1}$ of $C$, which will again become positively tangent at a point $L$ for the first time. In this way $a$ one-to-one continuous transformation $T$ of the ring into itself, taking any point $K$ into the corresponding point $L$, is obtained. The transformation $T$ may be so defined as to be continuous along the two periodic orbits for $C=C_{1}$ which form the boundaries of the ring, and varies continuously with $\mu$ from the transformation for $\mu=0$ obtained in $\mathbb{S}$ Io.

If, as in $\$$ IO, we represent the totality of orbits by the non-singular stream lines of a certain non-singular three-dimensional flow, a transformation of variables may be interpreted as a continuous deformation of these stream lines, and does not alter the nature of the dynamical problem. The ring given above may also be represented in this space; the points of the ring will correspond to the states of motion of the orbits at positive tangency whith an auxiliary curve of the ring. This ring may also undergo continuous deformation, each point of it being moved along the stream line on which it lies. By such deformations the transformation is readily seen to be essentially unaltered.

From this point of view the transformation $T$ determines the dynamical problem.

\section{$\int \mathrm{I} 4$.}

\section{Two Properties of the Transformation $T$.}

The fact that a volume integral remains invariant in the representing space (see $\$ \$ 2,6)$ is reflected directly in a fundamental property of the transformation $T:$ the transformation $T$ leaves a certain area integral $\iint \Phi(\rho, \theta) d \rho d \theta$ invariant where $\left.\Phi(p, \theta)>0^{{ }^{7}}\right)$.

This may be at once seen as follows: Consider the stream lines passing near an interior point $K$ of the ring represented in the three-dimensional manifold of states of motion. All of the stream lines will cut the ring in one and the same sense (cf. $\$$ ro) and will form a tube. Prolong this tube until the immediately following intersection of each stream line with the ring. Denote the area of one base of this tube by $\Delta A$ and the area of the other by $\Delta \bar{A}$. The points $(\bar{\rho}, \bar{\forall})$ of the second base are obtained from the points $(\rho, 9)$ of the first base by the transformation $T$.

Now let $t$ change to $t+\Delta t$ where $\Delta t$ is small. The tube of stream lines is transferred along itself to a new position. If we let $F(p, \theta)$ denote the rate at which a point moves along its stream line away from the ring at $(\rho, \theta)$ when $t$ changes, a point of either base will be approximately $F(\rho, \theta) \Delta t$ units away from the ring.

The invariant volume integrals taken over the tube between either base in its

17) H. Poincare, Les méthodes nouvelles de la Mécanique Céleste, vol. III (Paris, Gauthier-Villars, I 899), pp. 175-178. 
first and its displaced position must be equal. If $\iiint M d v$ ( $d v$ the element of volume) is the invariant volume integral, these two volumes are approximately

$$
M F(\rho, \theta) \Delta A \Delta t, \quad \bar{M} F(\bar{\rho}, \bar{\theta}) \Delta \bar{A} \Delta t
$$

where $M$ and $\bar{M}$ stand for the value of $M$ at a point of the first and second base respectively. By letting the dimensions of the base of the tube and the increment $\Delta t$ of time approach zero, we see that $\iint M F(\rho, \theta) d A$ taken over any part $\Sigma$ of the ring has the same value as when it is taken over the part $\overline{\mathbf{\Sigma}}$ into which $\mathbf{s}$ goes by the transformation $T$. This integral is the invariant area integral.

Furthermore, we may observe that $F(p, \theta)$ vanishes along the boundaries of the ring since the stream lines approach coincidence with the stream line which forms the boundary of the ring, and the rate $F(\rho, \theta)$ at which a point moves away from the ring is approaching zero.

Poincaré ${ }^{3}$ ) conjectured that a transformation $T$ of a ring possessing such an invariant integral left two points of the ring invariant if the coefficients of rotation were of opposite sign. Upon this basis $\left[\right.$ see $^{8}$ )] he was able to infer that there existed an infinite number of periodic solutions in the restricted problem of three bodies for $\mu$. sufficiently small.

But there is a second property of $T$ which, as will be seen later, may be made to yield an immediate proof that there exist an infinite number of symmetric periodic orbits, and upon which a classification of the symmetric orbits may be based:

The transformation $T$ is the product of two transformations $R, U$, each of period 2, of which the first is a reflection in the $x$-axis.

It is the fact that the equations of motion (1), (3) are unaltered when $x, y, t$ are replaced by $x,-y,-t$ respectively which leads to the above additional property.

This is immediately seen. Let $K$ be a point of positive tangency with one of the auxiliary orbits of the ring; let $R$ be the transformation by reflection in the $x$-axis which takes the ring into itself. We may denote by $L=T(K)$ the first following point of positive tangency (fig. 3). The positively tangent orbit at $L^{\prime}=R(L)$ will then be the

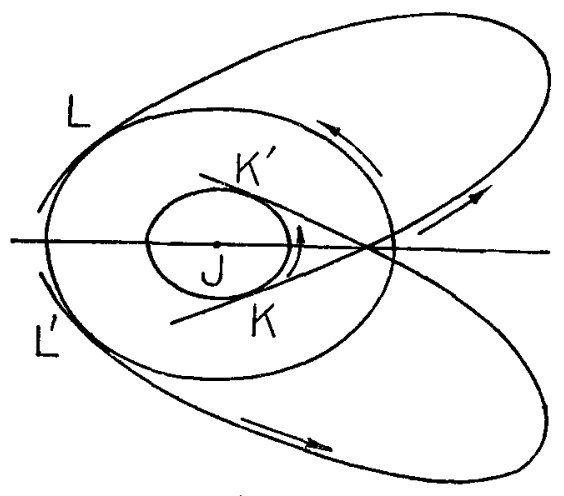

(Fig. 3). 
point of positive tangency of an orbit first tangent again at $K^{\prime}=T\left(L^{\prime}\right)=T R T(K)$. But a reflection brings $K^{\prime}$ into coincidence with $K$ on account of the symmetry noted. That is, we have

$$
R T R T=I
$$

where $I$ is the transformation which leaves every point invariant.

Hence the operation $R T$ is of period 2 and will be denoted by $U$. The transformation $R$ and its inverse are identical so that from $R T=U$ we get

$$
T=R U, \quad\left(R^{2}=U^{2}=I\right) \text {. }
$$

\section{$\int 15$.}

\section{On the Existence of a Transformation $T$.}

Suppose now that we postulate merely the existence of a retrograde periodic orbit $M$ and a direct periodic orbit $N$ about $J$ without double points or points of intersection. In the representing sphere these appear as two lines joining opposite points of its surface $(\$$ IO). If either of these lines be varied continuously into the other while its extremities remain always opposite, a ring is formed bounded by the two closed lines corresponding to the retrograde and direct periodic orbits.

The ring belonging to the transformation $T$ is precisely of this type, and is cut by every stream line in the same sense and infinitely often.

In the present paragraph we shall obtain a necessary and sufficient condition for the existence of a ring belonging to a transformation $T$ with these properties.

Let there be given any family of auxiliary retrograde curves of continuous curvature contracting from $M$ to a null curve at $J$, and a family of auxiliary direct curves expanding from this null curve to $N$. It will be assumed that the two families are without any positive tangency, and may be simultaneously deformed into two families of concentric circles about $J$ without introducing such a point of tangency. Furthermore it will be assumed that the small auxiliary curves approach a circular form about $J$ as center.

Let a positive tangency of an orbit to one of these curves be defined as exterior or interior according as the orbit lies outside of or inside of the curve at the point. The necessary and sufficient condition that a ring bounded by $M$ and $N$ shall exist in the representing space, cut by all the stream lines in the same sense and infinitely often, is that along every orbit the points of exterior tangency to the auxiliary curves exceed those of interior tangency in a sufficiently large fixed interval of time.

We shall begin by assuming that a family of auxiliary curves satisfying the conditions stated exists, and shall prove that this implies the existence of a ring transformation $T$.

With each point of an auxiliary curve let us associate the positively tangent state of motion. Then an auxiliary curve is represented by a line joining opposite points of 
the representing sphere, and the family of lines thus obtained corresponds to a ring limited by the lines which correspond to $M$ and $N$.

The manifold of states of motion for $\mu \neq 0$ may be continuously deformed into the like manifold for $\mu=0$, since both are equivalent to a sphere on which opposite points are taken as identical. This may be effected by deforming the region of motion for $\mu \neq 0$, into the circular region of motion for $\mu=0$, so that $J$ goes into itself, and by letting each state of motion correspond with the one with which it coincides in position and direction after the deformation.

Moreover the ring above obtained may deformed into the ring of $₫$ io for $\mu=0$ at the same time. In fact, we may take the family of direct auxiliary curves into the family of direct circular orbits for $\mu=0$ by continuous deformation, and likewise the family of retrograde auxiliary curves into the family of direct circular orbits. If it is assumed that each point of a curve is associated with the positively tangent state of motion, it is apparent that in this way we have defined a continuous deformation of the given ring into the ring of $\mathbb{S}$ ro for $y=0$. On account of the hypothesis about the auxiliary families of curves it is clear that no points of common positive tangency need be introduced during this deformation; consequently the same deformation of the ring may be effected by a continuous deformation of the representing space.

Hence we may represent the states of motion for the given $\mu$ by means of the space between two circular cylinders with common axis as in $\$ ro, so that $M$ and $N$ correspond to the surface of the outer and inner cylinder respectively, and so that the given ring of auxiliary curves is represented by a plane perpendicular to the axis. Furthermore the states of motion along $M$ and $N$ will correspond to spirals winding in opposite senses but toward the same end of the axis along the cylinders. Points at distance $2 \pi$ on one and the same line parallel to the axis of the cylinder represent the same state of motion.

The given ring divides the infinite cylinder into two parts which may be termed " upper» and "lower».

Take now two fixed points and join them by any line in the hollow cylinder. If the points are on the same side of the ring, the ring will necessarily be cut equally many times in either sense by such a line. If the points are on opposite sides of the ring there will be one more cutting in one sense than in the other.

Consider next a segment of a stream line. If $d$ be the distance between the two limiting planes perpendicular to the axis which include this segment between them, it is clear that only at most $d / 2 \pi$ rings congruent to the given ring cut this segment, and that the excess of total crossings of the first kind over total crossings of the second kind cannot exceed $d / 2 \pi$.

But our hypothesis ensures that along any orbit the number of points of exterior tangency to an auxiliary curve ultimately exceeds those at interior tangency by any prescribed integer. Exterior and interior tangency in the plane correspond, however, to the two senses of crossing of the ring by the stream line in our geometrical re- 
presentation. For, tangency to the ring in the representing space means equality of curvature. This fact may be established just as a similar fact has been in the case $\mu=o$ (cf. $\$$ Io). Also, over the direct or retrograde leaf of the ring taken separately, any exteriorly tangent curve may be continuously varied into any other through a series of such curves. To each such curve corresponds a line of the positively tangent states of motion in the representing space. Hence all of these lines cross in one and the same sense, so far as either leaf alone is concerned. But orbits near to $J$ are always exteriorly tangent to the auxiliary curves, since the curvature of the approximately parabolic orbit is about one half that of the approximately circular orbit at tangency. Hence one sense of crossing corresponds to one kind of tangency throughout. Thus after a sufficiently long interval of time the excess of crossings of one kind over those of the other becomes arbitrarily large.

When this excess has attained to $d / 2 \pi$, one can affirm that the terminal point of the stream line lies always at least $d$ units from the plane containing the initial point and perpendicular to the axis. It becomes apparent in this way that as $t$ increases from $-\infty$ to $+\infty$ all the points pass from the one end of the cylinder to the other along their stream lines.

Now let us take each stream line and continue it from $t=-\infty$ until it first cuts the ring. Remove all half stream lines of this kind which will lie in the first of the two parts into which the cylinder is divided. There will be exposed a ring-shaped surface made up of two kinds of regions: parts of the ring cut by a stream line in one and the same sense, and regions made up of segments of stream lines.

But any point of a boundary of the first kind of region is paired with a similar point of a similar region, namely the second point on the segment of the stream line forming part of the boundary and passing through the first point. By a distortion in which each point is moved along its stream line and paired points are made to coincide, a ring is obtained cut always by the trajectories in one and the same sense by every stream line. This ring has the desired properties and belongs to a transformation $T$.

It is readily seen that the stated condition is necessary. Let us again represent the states of motion by means of the space between two cylinders. We may assume that a ring exists belonging to a transformation $T$, and this ring may be taken as lying in a plane perpendicular to the axis of the cylinders.

But any family of curves having the properties stated will yield a second ring representing the states of motion at positive tangency to these curves. This ring may be deformed into the first plane ring. The stream lines which pass from one end of the cylinder to the other will cross this ring and its congruent images with increasing excess of crossings of one kind over those of the other. Since the two kinds of crossings correspond to the two kinds of tangency, this family of curves will satisfy the required conditions. 
PART IV.

PERIODIC ORBITS.

$\ 16$.

\section{Existence of Retrograde Periodic Orbits. Hill's Case.}

A question of fundamental importance for the restricted problem of three bodies concerns the existence of the periodic orbits of simplest type for all values of $\mu$ and $C$. This is also the problem whose solution is preliminary to the determination of the range of validity of the ring transformation. $U_{p}$ to the present time these orbits have only been considered for sufficiently small values of $\mu$ and for sufficiently large values of $C$.

I shall now pass on to establish that the retrograde periodic orbits do in fact always exist if there is a closed oval of zero velocity about $J$, and then by the aid of these results shall consider the more difficult problem afforded by the direct orbits. Let us begin with the limiting case of the restricted problem of three bodies treated by HILL [see $\left.{ }^{4}\right)$, which is especially simple.

The equations of motion in this case may be given the form

$$
\left\{\begin{array}{c}
\frac{d^{2} x}{d t^{2}}-2 \frac{d y}{d t}=x\left(3-\frac{1}{r^{3}}\right), \quad\left(r=\sqrt{x^{2}+y^{2}}\right), \\
\frac{d^{2} y}{d t^{2}}+2 \frac{d x}{d t}=-\frac{y}{r^{3}} \\
\left(\frac{d x}{d t}\right)^{2}+\left(\frac{d y}{d t}\right)^{2}=3 x^{2}+\frac{2}{r}-C .
\end{array}\right.
$$

In passing to this limiting case the units of length, mass and time have been altered. The body $J$ has been brought to the origin and is now of unit mass, the body $S$ has moved out indefinitely to infinity in the direction of the negative $x$-axis while at the same time the ratio of the mass $S$ to that of $J$ has indefinitely increased. The $x, y$-plane rotates with the bodies at unit angular velocity as before.

These equations enter into the category treated in $\$ 2$, with

$$
\lambda(x, y)=\mathrm{I}, \quad U(x, y)=\frac{3}{2} x^{2}+\frac{\mathrm{I}}{r}-\frac{\mathrm{I}}{2} C .
$$

If we apply the transformation

$$
x=p^{2}-q^{2}, \quad y=2 p q, \quad d t=4\left(p^{2}+q^{2}\right) d \tau,
$$

which is essentially that of Levi-Civita, the principles of $\ 2$ enable us to write the 
transformed equations at once

(53)

$$
\left\{\begin{array}{c}
\frac{d^{2} p}{d \tau^{2}}-8\left(p^{2}+q^{2}\right) \frac{d q}{d \tau}=V_{p}(p, q), \quad \frac{d^{2} q}{d \tau^{2}}+8\left(p^{2}+q^{2}\right) \frac{d p}{d \tau}=V_{q}(p, q), \\
\left(\frac{d p}{d \tau}\right)^{2}+\left(\frac{d q}{d \tau}\right)^{2}=2 V(p, q),
\end{array}\right.
$$

where

$$
V(p, q)=4\left[\frac{3}{2}\left(p^{2}-q^{2}\right)^{2}+\frac{1}{p^{2}+q^{2}}-\frac{1}{2} C\right]\left(p^{2}+q^{2}\right) .
$$

This form of equation is only necessary for our purposes in order to establish a simple fact, evident from physical considerations:

If the orbit of a particle $P$ passes through the neighborbood of $I$ there will be one and only one point $Q$ of this part of the orbit for which the distance from $J$ is a minimum. The orbit will be approximately a parabola with $Q$ as vertex and $I$ as focus. If the orbit is retrograde near $J$, it will be of loop form and cut the axis $Q J$ at points before and after $Q$, and near to $J$, at a small angle.

The existence of a unique point $Q$ and the approximately parabolic form of the orbit follow at once from the approximately rectilinear form in the $p, q$-plane, and the transformation employed.

We shall therefore pass at once to the proof of the loop form of the retrograde orbits. Here we shall confine attention to the typical case in which the point $Q$ lies along the $x$-axis. Under these circumstances the orbit cuts the $x$-axis at right angles. There will be two cases; in one case the point $Q$ lies to the left of $J$, and in the other to the right of $J$. Inasmuch as the treatment is the same in both cases we shall consider only the first, in which the particle is projected from a point $Q$ of the $x$-axis to the left of $J$ in the direction of the positive $y$-axis. We have merely to establish that the particle cuts the $x$-axis before and after projection at a small angle, near and to the right of $J$.

With the aid of the transformed differential equations a rigorous analytical proof is at once made. The transformation from the $x, y$-to the $p, q$-plane takes the positive $x$-axis into the positive $p$-axis, the negative $x$-axis into the positive $q$-axis, and the upper half of the $x, y$-plane into the first quadrant $p, q$-plane. The positive $y$-axis appears as the half line $p=q$ in this quadrant.

Since the transformation is conformal, the orbit in the $p, q$-plane begins at a point $\left(q_{0}, 0\right)$ near the origin on the positive $q$-axis with a velocity nearly $2 \sqrt{2}$ by the last equation (53). For $q_{0}=0$ the orbit in the $p, q$-plane starts from the origin in the direction of the positive $p$-axis. If this orbit lies at first below the $p$-axis, then, for $q_{\mathrm{o}}$ small and positive, the orbit will obviously cut the positive and negative $p$-axis near the origin in the $p, q$-plane at a small angle. The orbit will thus have the desired properties in the $x, y$-plane.

But for $q_{0}=0$ the initial conditions ate

$$
\frac{d q}{d \tau}=0, \quad \frac{d^{2} q}{d \tau^{2}}=0, \quad \frac{d^{3} q}{d \tau^{3}}=0, \quad \frac{d^{+} q}{d \tau^{4}}=-256 \sqrt{2}
$$


Hence $q$ is negatively immediately before and after projection i. e. the orbit in the $p, q$-plane does lie at first below the $p$-axis.

Thus a proof has been given under the stated circumstances.

The same considerations make it obvious that, in the limiting cusped form, which corresponds to collision, the particle $P$, as seen from $J$, lies to the left of the axis of the cusp before collision, and to the right of that axis after collision.

For our present purposes we shall restrict attention to the case $C>3^{\frac{4}{3}}$. In this case only there will be a closed oval,

$$
3 x^{2}+\frac{2}{r}-C=0,
$$

about $J$, symmetric with respect to both axes, in which motion is possible and along whose boundary the velocity is zero. It is easily seen that this oval lies within a circle of radius $3^{-\frac{1}{3}}$ about $J$ as center; for if we have $r=3^{-\frac{1}{3}}$, the left-hand side of the above equation is necessarily negative.

Let us suppose now that the particle is projected at any point $K \equiv\left(x_{0}, 0\right)$ to the left of the origin at right angles to the $x$-axis and toward the positive $y$-axis; let $x, y$ denote the coördinates of the point at the time $t$, and let $t=0$ be the time of projection.

From the first equation $(52)$ we obtain

$$
\frac{d x}{d t}-2 y=\int_{0}^{t} x\left(3-\frac{\mathrm{I}}{r^{3}}\right) d t,
$$

by integration between the instant $t=0$ of projection and any value of $t$. As long as $x$ is negative the integral on the right is a positive increasing function of $t$. Therefore while $y \gg 0$, the velocity $d x / d t$ will exceed a positive increasing quantity. Consequently a time $\tau$ may be assigned in advance such that either $y=0$ or $x=0$ for some $t<\tau$. Let the corresponding point of the orbit be $L$; the point $L$ lies on the negative $x$-axis or the positive $y$-axis; along $K L$ we have $d x / d t>0$.

It is clear that for $K$ near to $f$, the point $L$ lies on the $y$-axis, and that the direction of the orbit at $L$ is never parallel to the $y$-axis.

As $K$ moves to the left along the $x$-axis to its extreme position, the point $L$ varies continuously as long as no new root $y=0$ appears along the segment $K L$, and $L$ does not approach the origin $J$.

But a root $y=0$ cannot appear within $K L$ for at the same point we should have $d x / d t>0, d y / d t=0, d^{2} y / d t^{2} \supseteq 0$, which is impossible by the second equation $(52)$.

In consequence the point $L$ and the segment $K L$ vary continuously with $K$, at least until $L$ coincides with $J$.

As the abscissa $x_{0}$ of the point of projection diminishes from zero to its extreme negative value, the point $K$ moves from the origin to the oval of zero velocity along the negative $x$-axis. At the outset $d y / d t$ is positive at $L$, since the approximately para- 
bolic orbit cuts the $y$-axis at a slope approximately $\mathrm{I}$. At the terminal position of $K$ on the oval of zero velocity we have

$$
y=\mathrm{o}, \quad \frac{d y}{d t}=\mathrm{o}, \quad \frac{d^{2} y}{d t^{2}}=\mathrm{o}, \quad \frac{d^{3} y}{d t^{3}}<\mathrm{o},
$$

from the differential equations (52). The particle is projected at velocity zero and moves below the axis in this case; the arc $K L$ bas necessarily passed through $J$ earlier.

There is thus an intermediate position of $K$ at which $L$ lies at the origin for the first time, and the orbit has cusp form with the axis of the cusp in the second quadrant $(x \leqslant 0, y>0)$. In the nearby approximately parabolic form the axis of the parabola is essentially in the same direction as that of the cusp. Bearing this in mind we see that in any possible position of the axis the slope of the orbit at $L$ lies between $-\infty$ and - I approximately (fig. 4 ). This fact may be proved at once by

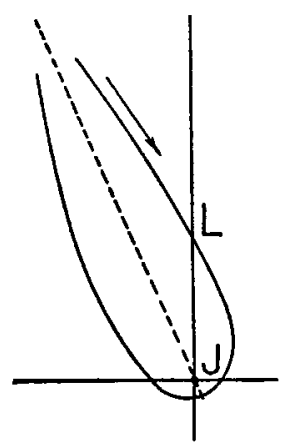

(Fig. 4).

a transformation to the variables $p, q, \tau$. Hence, previous to the passage of $L$ through the origin, the arc $K L$ cuts the $y$-axis with negative slope.

That is, $d y / d t$ at $L$ has changed from positive to negative as $K$ passes from the origin to the position for which $L$ coincides with the origin.

At some intermediate position we will have $d y / d t=0$, and $K L$ cuts the $y$-axis as well as the $x$-axis perpendicularly.

The differential equations remain unchanged if $x, y, t$ be replaced by $x,-y,-t$ or $-x, y,-t$ respectively. Therefore $K L$ with its successive reflections in the $x$ and $y$-axes constitutes a complete retrograde periodic orbit.

Now we note that $d y / d t$ does not vanish between $K$ and $L$. For, at one of two successive roots of $d y / d t=0$, we have necessarily $d^{2} y / d t^{2} \supseteq 0$ which is impossible by the second equation ( 52 ) when $d x / d t>0$.

In the limiting case of the restricted problem of three bodies treated by HILL, there exists for $C>3^{\frac{4}{3}}$ a retrograde periodic orbit which makes a single circuit of $J$ within the oval of zero velocity about $J$, which is symmetric with respect to both axes and such that $d x / d t, d y / d t$ do not vanish except along the intersection with the $x$ - and $y$-axes respectively.

The expression for the curvature along the orbit together with the facts just stated make it obvious that the oval formed by the orbit is convex. 
It is clear that the nature of the preceding argument is largely independent of the restriction $C>3^{\frac{4}{3}}$.

\section{SI7.}

Some Properties of the Curves $\Omega_{x}(x, y)=0$ and $\Omega_{y}(x, y)=0$.

In order to generalize the argument of the preceding paragraph we need to know the nature of the part of the curves $\Omega_{x}(x, y)=0, \Omega_{y}(x, y)=0$ which lies within the oval of zero velocity about $J$. For the liniting case of $\$ 16$ these curves reduce to the $y$ - and $x$-axes respectively.

The simpler equation to consider is $\Omega_{y}(x, y)=0$ or

$$
y\left(\mathrm{I}-\frac{\mathrm{I}-\mu}{r_{\mathrm{I}}^{3}}-\frac{\mu}{r_{2}^{3}}\right)=0 .
$$

There are no branches of this curve other than $y=0$ within the oval. This is obvious in the case $\mu=0$. Since the oval does not pass through a point

$$
\mathbf{\Omega}_{\boldsymbol{x}}(x, y)=\mathbf{\Omega}_{y}(x, y)=\mathrm{o}
$$

(see $₫ 7$ ), the equation $\boldsymbol{\Omega}_{y}(x, y)=0$ is the condition that the tangent to the oval be parallel to the $y$-axis. If an additional root of this equation along an oval about $J$ be introduced as the ovals vary with $\mu$, it will correspond to a point of inflection with vertical tangent (i. e. a tangent parallel to the $y$-axis).

A point of inflection cannot lie along the $x$-axis unless $\Omega_{y y}(x, y)$ vanishes along that axis; and this condition implies that the coefficient of $y$ in (55) vanishes along the $x$-axis within the oval:

$$
\mathrm{I}-\frac{\mathrm{I}-\mu}{|x-\mu|^{3}}-\frac{\mu}{|x+\mathrm{I}-\mu|^{3}}=0 .
$$

This is not possible. In the first place if the point $(x, 0)$ lies between $S$ and $J$ both of the denominators in the left-hand member of this last equation are numerically less than I. The two fractions are greater than I $-\mu$ and $\mu$ respectively in consequence, and the left-hand member is negative. To treat the possibility $x>\mu$ or $x<-\mathrm{I}+\mu$, we note that $\Omega_{x}(x, y)$ may be written in the alternative forms [see (26)]

$$
\left\{\begin{array}{c}
(x-\mu)\left(I-\frac{I-\mu}{r_{1}^{3}}-\frac{\mu}{r_{2}^{3}}\right)+\mu\left(I-\frac{I}{r_{2}^{3}}\right) \\
\text { or }(x+I-\mu)\left(I-\frac{I-\mu}{r_{1}^{3}}-\frac{\mu}{r_{2}^{3}}\right)-(I-\mu)\left(I-\frac{I}{r_{1}^{3}}\right) .
\end{array}\right.
$$

The first of these two forms shows us that $\Omega_{x}(x, y)$ reduces to $\mu \cdot\left(\mathrm{I}-\mathrm{I} / r_{2}^{3}\right)$ at a point of inflection along the $x$-axis, and is therefore positive for $x>\mu$. But $\Omega_{x}(x, y)$ is negative for $x-\mu$ positive and small, and first vanishes at $P_{\text {; }}$ (see $\ 6$ ). The possibility $x>\mu$, and likewise $x<-\mathrm{I}+\mu$, is accordingly to be excluded.

At a point $y>0$ of the oval a vertical point of inflection arises also if the 
equations $\Omega_{y}(x, y)=0, \Omega_{y y}(x, y)=0$ are simultaneously satisfied. Hence the partial derivative of $\Omega_{y}(x, y) / y$ as to $y$ will vanish. But this ratio is the coefficient of $y$ in the left-hand member of (55), and the partial derivative of this coefficient as to $y$ consists of two positive terms. Hence a point of inflection for $y>0$ cannot exist.

If now we observe that in the vicinity of $J$ for $y>0$ the function $\Omega_{y}(x, y)$ is negative, we can conclude that the function $\Omega_{y}(x, y)$ vanishes only along the line $y=0$ in the oval of zero velocity about $J$, and is negative for $y>0$ in that oval.

Considerations of symmetry establish that $\Omega_{y}(x, y)$ is also negative in the part $y>0$ of the oval about $S$.

The second equation to be considered is $\boldsymbol{\Omega}_{x}(x, y)=0$, and may be written

$$
x-\frac{(\mathrm{I}-\mu)(x-\mu)}{r_{1}^{3}}-\frac{\mu(x+\mathrm{I}-\mu)}{r_{2}^{3}}=0 .
$$

If we employ the first alternative form $(56)$ for $\mathbf{\Omega}_{x}(x, y)$ we see that this function is positive in the part of the oval about $J$ between the line $x-\mu=0$ and the circle $r_{2}=\mathrm{I}$; for, this is the region $r_{2}>\mathrm{I}, x-\mu<0$ and the coefficient of $x-\mu$ is $\boldsymbol{\Omega}_{y}(x, y) / y<0$. Likewise from the second alternative form we see that $\boldsymbol{\Omega}_{x}(x, y)$ is negative in the part of the oval about $S$ between the line $x+1-\mu=0$ and the circle $r_{1}=\mathrm{I}$.

As the constant $C$ of JACOBI diminishes from $+\infty$ it is clear that at first $\mathbf{\Omega}_{x}(x, y)$ vanishes at two and only two points along the oval $\Omega(x, y)-\frac{1}{2} C=0$ which is approximately a small circle about $J$ as center (see $\$ 7$ ); these points will be the points at which the tangent to the oval is parallel to the $x$-axis, and are symmetrically placed with respect to that axis. A single curve branch of the curve $\Omega_{x}(x, y)=0$ must therefore pass through $J$, with a vertical tangent.

It has already been noted that for $x>\mu$ and $y=0$ the first root of $\boldsymbol{\Omega}_{x}(x, y)=0$ lies at $P_{3} \equiv\left(x_{3}, 0\right)$ outside the oval, and that $\Omega_{x}(x, y)$ is negative along the part of the $x$-axis in the oval. As $y$ increases from zero, $\Omega_{x}(x, y)$ increases, since we have

$$
\mathbf{\Omega}_{x y}(x, y)=\frac{3(\mathrm{I}-\mu)(x-\mu) y}{r_{\mathrm{I}}^{5}}+\frac{3 \mu(x+\mathrm{I}-\mu) y}{r_{2}^{5}} .
$$

Now for any positive $x$ and for large enough $y$, the function $\Omega_{x}(x, y)$ is positive. Consequently there is one and only one point on the curve $\boldsymbol{\Omega}_{x}(x, y)=0$ for a given $\mu<x<x_{3}$ above the $x$-axis. In this way we obtain a closed branch of this curve which will of course be symmetric with respect to the $x$-axis.

Any radial line from $J$ will cut this branch in one and only one point. Otherwise a radial line will be tangent to the curve at some point. This implies

or

$$
\begin{gathered}
y \mathbf{\Omega}_{x y}(x, y)+(x-\mu) \boldsymbol{\Omega}_{x x}(x, y)=0, \\
(x-\mu)\left[\mathrm{I}+\frac{2(\mathrm{I}-\mu)}{r_{\mathrm{I}}^{3}}+\frac{2 \mu}{r_{2}^{3}}\right]+\frac{3 \mu y^{2}}{r_{2}^{5}}=0,
\end{gathered}
$$

which is not possible for $x>\mu$. 
There is a closed branch of the curve $\Omega_{x}(x, y)=0$, within the oval of zero velocity about $J$ and lying in the strip $\mu \leq x \leq x_{3}$, intersected by each line parallel to the $y$-axis within the strip, in two points symmetrically placed with respect to the $x$-axis. This branch passes through $J$ vertically but through no other point of the $x$-axis uithin the oval, and is cut by a radial line from $J$ in one and only one point. The function $\Omega_{x}(x, y)$ is negative to the right of this branch and positive to the left of this branch.

Considerations of symmetry establish that there is a similar branch through $S$ to the left of the line $x=-\mathrm{I}+\mu$.

I shall now pass on to prove that this branch of the curve $\mathbf{\Omega}_{x}(x, y)=0$ is the only branch within the oval of zero velocity about $J$.

For $x<-\mathrm{I}+\mu$ and $x>\mu$. we have already excluded the possibility of additional branches in the ovals about $S$ and $J$ beyond those already obtained. Furthermore we have noted that $\Omega_{x}(x, y)$ is positive for $x<\mu, r_{2}>\mathrm{I}$ and negative for $x>-\mathrm{I}+\mu$, $r_{\mathrm{r}}>\mathrm{I}$. Hence we need only consider the region $r_{1} \leq \mathrm{r}, r_{2} \leq \mathrm{r}$ which will contain all further parts of the curve $\Omega_{x}(x, y)=0$ which can lie within the oval about $S$ or $J$.

For $\mu=\mathrm{o}$ we have the limiting branch $r_{1}=\mathrm{I}$ in this region, and for $\mu=\mathrm{I}$ we have the limiting branch $r_{2}=\mathrm{I}$ [see (57)]. In each intermediate position the branch passes through $r_{1}=r_{2}=\mathrm{I}$ i. e. through $P_{4}$. For $\mu=\frac{1}{2}$ we have a branch $x=0$.

Moreover for any $\mu$ we have one and only one point of a branch $\boldsymbol{\Omega}_{x}(x, y)=0$ along $S J$, namely the point $P_{2}$ corresponding to the particular $\mu$ (see $\$ 6$ ), and this point varies from $S$ to $J$ as $\mu$ varies from o to $\mathrm{I}$.

Now if we make the change of variables $x-\mu=\bar{x}, y=\bar{y}$ and in this way transfer the origin to $J$ for any $\mu$, the equation $\boldsymbol{\Omega}_{x}(x, y)=0$ becomes linear in $\mu$. Consequently each point of the region $r_{1} \leq \mathrm{I}, r_{2} \leq \mathrm{I}$ in the $\bar{x}, \bar{y}$-plane lies on a branch $\Omega_{x}(x, y)=\mathrm{o}$ for at most one value of $\mu$.

Neglecting certain details of demonstration, we conclude that as $\mu$ varies from $o$ to $\mathrm{I}$, the branches of $\Omega_{x}(x, y)=0$ in the $\vec{x}, \vec{y}$-plane form a regular pencil of curves through $r_{1}=r_{2}=\mathrm{I}$ varying from $r_{1}=\mathrm{I}$ to $r_{2}=\mathrm{I}$, passing through each point of the region under consideration once and only once. The curves are clearly symmetric with respect to the $\bar{x}$-axis and intersect it perpendicularly.

In this way we have proved that there exists one and only one branch of $\Omega_{x}(x, y)=0$ in the region $r_{1} \leq \mathrm{I}, r_{2} \leq \mathrm{I}$, and have determined its general character. It remains to prove that this branch is exterior to the ovals about $S$ and $J$ for any value of $\mu$.

Let us pass circles with $S$ and $J$ as centers through $P_{2}$ and let their radii be $\rho_{2}$ and $\rho_{\mathrm{I}}$ respectively. We shall establish that for any $\mu$ the branch of $\Omega_{x}(x, y)=0$ is exterior to the circles, and that the ovals about $S$ and $J$ lie wholly within them for $r_{1} \leq \mathrm{I}, r_{2} \leq \mathrm{I}$, and thus complete the proof.

In the first place if the branch lies partly within one of these ovals, the one about $J$ for instance, it will necessarily intersect the circle about $J$ through $P_{2}$, in another point $Q$, since the terminal point $P_{4}$ lies outside of this circle. At this point 
we have $r_{1}=p_{1}$ by definition of $p_{1}$, and the condition $\Omega_{x}(x, y)=0$ may be written

$$
(\mathrm{I}-\mu)(x-\mu)\left(\mathrm{I}-\frac{\mathrm{I}}{\rho_{\mathrm{I}}^{3}}\right)+\mu(x+\mathrm{I}-\mu)\left(\mathrm{I}-\frac{\mathrm{I}}{r_{2}^{3}}\right)=0 .
$$

Also at $P_{2}$ we have $\mu-x=r_{1}=p_{1}, x+\mathrm{I}-\mu=r_{2}=p_{2}$ so that similarly

$$
-(I-\mu) p_{1}\left(I-\frac{I}{P_{1}^{3}}\right)+\mu p_{2}\left(I-\frac{I}{p_{2}^{3}}\right)=0 .
$$

Comparing these equations we obtain

$$
\frac{x+\mathrm{I}-\mu}{\mu-x}\left(\frac{\mathrm{I}}{r_{2}^{3}}-\mathrm{I}\right)=\frac{\mathrm{P}_{2}}{\mathrm{P}_{\mathrm{I}}}\left(\frac{\mathrm{I}}{\mathrm{P}_{2}^{3}}-\mathrm{I}\right)
$$

it is to noted that $x+\mathrm{I}-\mu$ and $\mu-x$ are positive at $Q$. Now the left-hand side of this equation will be increased if $r_{2}$ is replaced by the smaller quantity $x+1-\mu$. By doing this and at the same time replacing $p_{1}$ by its equal $I-p_{2}$ in the right-hand side, we deduce the inequality

$$
\frac{\mathrm{I}}{(x+\mathrm{I}-\mu)^{2}}+\frac{\mathrm{I}}{(x+\mathrm{I}-\mu)}+\mathrm{I}>\frac{\mathrm{I}}{\mathrm{P}_{2}^{2}}+\frac{\mathrm{I}}{\mathrm{P}_{2}}+\mathrm{I} .
$$

But we have also $x+\mathrm{I}-\mu>p_{2}$ at $Q$ since every point on the circle about $J$ lies to the right of $P_{2}$. Therefore this inequality is impossible.

It remains to prove that the ovals of zero velocity are interior to these circles in the region $r_{1} \leq \mathrm{I}, r_{2} \leq \mathrm{I}$.

The least value $C_{o}$ of the constant $C$ of JACoBr yielding an oval of the type under consideration is $2 \Omega\left(x_{2}, 0\right)$ which corresponds to the limiting ovals through $P_{2}$. The ovals to be considered lie in the region $2 \Omega(x, y)-C_{0} \gg 0$. This inequality may be written

$$
(\mathrm{I}-\mu)\left(r_{1}^{2}+\frac{2}{r_{1}}-p_{1}^{2}-\frac{2}{i_{1}}\right)+\mu\left(r_{2}^{2}+\frac{2}{r_{2}}-\rho_{2}^{2}-\frac{2}{p_{2}}\right) \supseteq 0 .
$$

Outside of the two circles of radii $\rho_{2}$ and $\rho_{1}$ about $S$ and $J$ respectively in the region under consideration, both $r_{1}$ and $r_{2}$ are increased beyond their values $p_{1}$ and $p_{2}$ at $P_{2}$. Both terms of the left-hand member of this inequality ate thereby diminished, and become negative, since $r_{1}$ and $r_{2}$ are less than I. In consequence this inequality cannot hold outside of these circles.

The proof is now complete.

$$
\text { \18. }
$$

\section{Existence of Retrograde Orbits. General Case.}

We are now in a position to establish the existence of a retrograde periodic orbit for the general restricted problem of three bodies under the hypothesis that for the given value of $C$ the particle lies within a closed oval of zero velocity about $J$.

We shall prove first: If the particle $P$ within the oval of zero velocity about $J$ be 
projected from the $x$-axis to the left of $J$ in the direction of the positive $y$-axis, the condition $d x / d t>0$ will hold at least until one of the conditions $\Omega_{x}(x, y)=0$ or $y=0$ is satisfied.

To prove this we observe first that so long as $\Omega_{x}(x, y)$ is positive $(\$ 17)$ we have by the first equation (I)

$$
\frac{d^{2} x}{d t^{2}}-2 \frac{d y}{d t}>0
$$

Integrating between the time $t=0$ of projection at $K$, when we have $d x / d t=y=0$, and the time $t$, we find

$$
\frac{d x}{d t}-2 y>0
$$

so that $d x / d t$ remains positive as long as $y \supseteq 0$ and $\Omega_{x}(x, y) \supseteq 0$.

Thus by $\$ I 7 the orbit satisfies the condition $d x / d t>0$ either until a point $L$ for which $x-\mu=0, y \geqslant 0$ is reached, or until we have $y=0, x-\mu<0$; it is clear that one of these alternatives actually arises, since we cannot have $d x / d t>0$ permanently. For $K$ near to $J$ there is such a point $L$.

Let $\tau(-\pi / 2<\tau<\pi / 2)$ denote the angle which the direction of motion at $L$ makes with the positive $x$-axis. As $K$ varies from $J$ to its limiting position on the oval of zero velocity, the point $L$ will also vary in such a way that the angle $\tau$ and the ordinate $y$ at $L$ vary analytically. As has been seen above, this variation can only terminate if either $y$ becomes zero at an interior point of $K L$, or if $L$ coincides with $J$. But the first possibility may be excluded, inasmuch as the conditions $d x / d t>0$, $d^{2} y / d t^{2} \geq 0$ are incompatible with the second equation (I) by $₫ 17$. Hence the variation will actually terminate by coincidence of $L$ with $J$, for in its limiting position on the left the projected particle falls at once below the $x$-axis ( $f$. $\ 16$ ).

If we recall that for $K$ near to $J$ the value of $\tau$ is nearly $\pi / 4$ on account of the form of the orbit $\left(S_{1} 6\right)$, and note that in the terminal position, the axis of the final cusped orbit is inclined at an angle $\theta(\pi / 2 \leq \theta<\pi)$ to the positive $x$-axis, we can infer that the curve $\Gamma$ representing the values of $\tau, y$ in rectangular coördinates is a continuous curve lying wholly inside of the rectangle $-\pi / 2<\tau<\pi / 2,0 \leqslant y<y_{0}$ 。 ( $y_{\mathrm{o}}$ being the ordinate of the point with abscissa $x=\mu$. on the oval of zero velocity about $J$ ), except for an initial point $(\pi / 4,0)$ and a terminal point $(-\pi / 4-d, 0)$ where $0<d \leqslant \pi / 4$ (fig. 5).

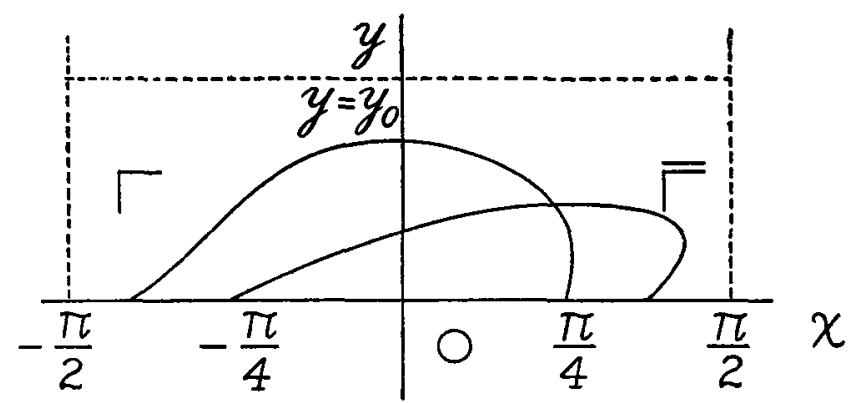

(Fig. 5). 
The equality signs have been excluded on account of the character of the retrograde cusped orbit at $J$; the particle before collision lies to the left of the axis of the cusp as seen from $J$. A rigorous consideration of the nature of the orbit near collision is easily made precisely as in $\ \mathrm{I} 6$, by a use of the equations of Levi-Civita, and leads to the same conclusions.

We shall now pass to the consideration of the orbits terminating at a point $\bar{K}$ on the $x$-axis to the right of $J$, and shall prove: If the particle $P$ be projected to $a$ terminal position $\bar{K}$ on the $x$-axis to the right of $J$ in the direction of the negative $y$-axis, the condition $d x / d t>0$ will hold before $\bar{K}$, at least until one of the conditions $\Omega_{x}(x, y)=0$ or $y=0$ is satisfied. If $\bar{K}$ is sufficiently near to $J$, the conditions $d x / d t>0, y>0$ will hold until an earlier instant for which $x-\mu$ becomes negative.

Here we observe that, as long as $\Omega_{x}(x, y)$ is negative $(\$ I 7)$, we have by the first equation (I)

$$
\frac{d^{2} x}{d t^{2}}-2 \frac{d y}{d t}<0
$$

Integrating between the time $t$ and the terminal time $t=0$ when we have $d x / d t=y=0$ we conclude that

$$
\frac{d x}{d t}-2 y>0
$$

as before. If now we recall the approximately retrograde parabolic form of orbit for $\bar{K}$ near to $J$, with $J$ as focus and $\bar{K}$ as vertex, the truth of the last italicized statement becomes manifest also.

Let now $\bar{L}$ denote the first earlier point of the orbit ending at $\bar{K}$ for which $x-\mu$ reduces to zero. The point $\bar{L}$ will certainly exist and will vary analytically with $\bar{K}$, at least for $\bar{K}$ near to $J$. In fact it is apparent that it will vary in this way so long as the conditions $d x / d t>0, y>0$ continue to hold along $\widetilde{L} \bar{K}$. We shall denote the angle which the direction of motion at $\bar{L}$ makes with the positive $x$-axis by $\tau$, and the ordinate of $\bar{L}$ by $y$. Also we shall let $\bar{\Gamma}$ denote the curve formed by the set of values $\tau, y$ when $\bar{K}$ is varied from $J$ to the extreme position for which the conditions above enumerated hold.

It will be proved that the curve $\bar{\Gamma}$ necessarily intersects the curve $\Gamma$ (fig. 5).

This is apparent if the extreme position of $\bar{K}$ is one for which we have a collision at $J$. For in this case by the preceding methods we may establish that $\bar{\Gamma}$ is a closed curve lying within the rectangle in which $\Gamma$ lies save for an initial point $(-\pi / 4,0)$ and a terminal point $(\pi / 4+e, 0)$ where $o<e \leqslant \pi / 4$ (fig. 5). The curve $\overline{\boldsymbol{\Gamma}}$ extends from a point on one side of the curve $\mathbf{\Gamma}$ to a point on the other side.

We have further to consider the case for which $L$ remains distinct from $J$ during the period of variation while, however, at the terminal point of $\bar{\Gamma}$, some point of $\bar{L} \vec{K}$ satisfies either the condition $y=0$, or the condition $d x / d t=0$.

The possibility $y=0$ may be excluded, for at such a point we must have si- 
multaneously $d x / d t>0, d^{2} y / d t^{2} \geqslant 0$ which is in contradiction to the second equation (I) (see $\int \mathrm{I} 7$ ).

Hence we may restrict attention to the possibility $d x / d t=0, y>0$ at some point of $\bar{L} \bar{K}$. We may exclude the point $\bar{K}$ as not one for which a further root $d x / d t=0$ appears, sinie the first equation (I) establishes that $d^{2} x / d t^{2}$ is negative at $\bar{K}$.

If this point is $\bar{L}$, the terminal value of $\tau$ is $\pm \pi / 2$, and the curve $\bar{\Gamma}$ extends from the point $(-\pi / 4,0)$ on one side of the curve $\Gamma$ to a point $( \pm \pi / 2, y)$ on the other side of $\Gamma$ (fig. 5). The curves $\Gamma$ and $\overline{\mathbf{r}}$ will intersect in this case also.

It is, however, impossible for the conditions $d x / d t=0, y>0$ to be satisfied first at an interior point $M$ of $\bar{L} \bar{K}$. If this impossibility be established it is clear that $\mathbf{r}$ and $\overline{\boldsymbol{r}}$ always intersect.

In order to establish it we make a division of cases according as $d y / d t>0$ or $d y_{i}^{\prime} d t \leq \mathrm{o}$ at $M$, the first of which I shall now consider.

Let us multiply the first equation (I) by $y$, the second by $x-\mu$ and subtract the two resulting equations, member for member, from each other. We shall thus obtain

$$
y \frac{d^{2} x}{d t^{2}}-(x-\mu) \frac{d^{2} y}{d t^{2}}-2(x-\mu) \frac{d x}{d t}-2 y \frac{d y}{d t}=\mu\left(\mathrm{I}-\frac{\mathrm{I}}{r_{2}^{3}}\right) y .
$$

If $r_{1}$ has the same meaning as before, and if $\theta_{1}$ is the angle which the line $J P$ makes with the $x$-axis, this equation may be written

$$
\frac{d}{d t}\left(-r_{\mathrm{1}}^{2} \frac{d \theta_{1}}{d t}-r_{\mathrm{I}}^{2}\right)=\mu\left(\mathrm{I}-\frac{\mathrm{I}}{r_{2}^{3}}\right) y .
$$

At $\bar{L}$ on the line $x-\mu=0$ it is clear that $d \theta_{1^{\prime}}{ }^{\prime} d t<0$, while at $M$ it is clear that $d \theta_{1} / d t>0$. There is thus a point $N$ on $\bar{L} M$ for which $d \theta_{1} / d t=0, d^{2} \theta_{1} / d t^{2} \gg 0$. Also from the equation $x-\mu=r_{1} \cos \theta_{1}$ we infer that the condition

$$
\frac{d x}{d t}=-r_{1} \sin \theta_{1} \frac{d \theta_{1}}{d t}+\frac{d r_{1}}{d t} \cos \theta_{1} \supseteq 0
$$

holds at such a point, from which we have accordingly $d r_{v^{\prime}}^{\prime} d t \supseteq 0$.

But the right-hand member of (59) is positive at $N$, while the left-hand member reduces to $-r_{1}^{2} d^{2} \theta_{1} / d t^{2}-2 r_{1} d r_{1} / d t$ which is negative or zero at $N$, so that a contradiction has been reached in this case.

In the case where we have $d x / d t=0, y>0$ and $d y / d t \leqslant 0$ at an interior point $M$ of $\bar{L} \bar{K}$ we make use of the conditions $d^{2} x / d t^{2}=0, d^{3} x / d t^{3} \supseteq 0$ which must clearly obtain since the condition $d x / d t \supseteq 0$ holds along $\bar{L} \bar{K}$. From the differential equations ( $\mathrm{I}$ ) we infer

$$
\frac{d^{3} x}{d t^{3}}=2\left(-2 \frac{d x}{d t}+\Omega_{y}(x, y)\right)+\Omega_{x x}(x, y) \frac{d x}{d t}+\Omega_{x y}(x, y) \frac{d y}{d t} .
$$

At $M$ we find therefore that $d^{3} x / d t^{3}$ reduces to

$$
2 \Omega_{y}(x, y)+\boldsymbol{\Omega}_{x y}(x, y) \frac{d y}{d t} .
$$


Since $\boldsymbol{\Omega}_{y}(x, y)$ is negative for $y>0$ and since $d y / d t$ is negative or zero, it follows that $\Omega_{x y}(x, y)$ must be negative if $d^{j} x / d t^{3}$ is to be positive or zero. This is not possible for from $(58) \Omega_{x y}(x, y)$ is positive at $M$.

Thus the curves $\vec{i}$ and $\overline{\mathbf{r}}$ obtained will necessarily intersect at one or more points.

If the particle $P$ passes through the corresponding point $Q$ of the line $x=\mu$ with the proper value of $\tau$, its orbit before the point is reached constitutes an arc $K L$, and its orbit after the point is reached constitutes an arc $\bar{L} \bar{K}$. These two arcs together form a retrograde half oval about $J$ along which $d x / d t$ is positive, intersecting the $x$-axis perpendicularly at $K$ and $\bar{K}$, and becoming parallel to the $x$-axis only once; in fact if there were more than one point $d y / d t=0$, the conditions $d^{2} y / d t^{2} \geqslant 0$, $d x / d t>o$ would hold at one of them, which is impossible by the first equation (I).

Since the differential equations of motion are unchanged if $x, y, t$ be replaced by $x,-y,-t$ respectively, we conclude that the arc $K \bar{K}$ thus obtained, together with its reflection in the $x$-axis, constitutes a retrograde symmetric periodic orbit.

For any value of $C$ for which there is a closed oval of zero velocity about $J$, there will exist at least one periodic orbit making a single retrograde circuit of $J$, symmetric with respect to the $x$-axis, with tangents parallel to the $y$-axis at two points of the $x$-axis, and with tangents parallel to the $x$-axis at two symmetrical points.

It is obvious that similar methods will lead to a proof of the existence of retrograde periodic orbits in more general cases.

\section{\I9.}

\section{An Alternative Form of Transformation. Direct Orbits.}

We shall now return briefly to the case $\mu=0$ in order to establish the fact that it is possible to make a reduction of the restricted problem of three bodies to the transformation of a discoid (by a discoid I mean a two-dimensional region deformable into a circle) into itself.

When $\mu$ is zero, the parameters $a, \theta, \psi(\$ 9)$ serve to represent the states of motion for a given $C$. These parameters were taken as the cylindrical coördinates of a point in space. The totality of states of motion was then represented by the points of a hollow cylinder $a_{1} \leq a \leq a_{2}, 0 \leq \psi \leq 2 \pi$ where opposite points of the bases $\psi=0$ and $\psi=2 \pi$ were taken as identical by convention.

It is convenient for us to replace the variables $a, \theta, \psi$ by

$$
\bar{a}=\frac{\mathrm{I}}{a}-\frac{\mathrm{I}}{a_{2}}, \quad \bar{\theta}=\theta, \quad \bar{\psi}=\psi-\theta
$$

where $\bar{a}, \vec{\theta}, \bar{\psi}$ are again taken as cylindrical coördinates. The region of representation is now the ordinary cylinder $\bar{a} \leq\left(a_{2}-a_{1}\right) / a_{1} a_{2}, 0 \leq \bar{\psi} \leq 2 \pi$ under a similar convention.

In the $a, \theta, \psi$ representation the outer and inner cylindrical surfaces correspond 
to the direct and retrograde circular orbits respectively; the spirals $\theta+\psi=$ constant and $\theta-\psi=$ constant on these respective surfaces correspond to the individual states of motion.

It the $\bar{a}, \bar{\theta}, \bar{\psi}$ representation the surface of the outer cylinder corresponds to the retrograde circular orbit and the spirals $2 \overline{\boldsymbol{\theta}}+\bar{\psi}=$ constant on that cylinder correspond to the individual states of motion along the orbit. The axis $\bar{a}=0$ of the cylinder corresponds to the direct circular orbit, and to an individual state along that orbit corresponds the point $\bar{a}=0, \bar{\psi}=$ constant.

Here then the parametric representation is only singular along the surface of the outer cylinder which corresponds to the retrograde circular orbit.

By (37) the orbits will have the equations

$$
\bar{a}=\bar{a}_{0}, \quad \bar{\theta}=-t+\bar{\theta}_{0}, \quad \bar{\psi}=\left[\left(\frac{a_{2} \bar{a}_{0}+\mathrm{I}}{a_{2}}\right)^{\frac{3}{2}}+\mathrm{I}\right] t+\bar{\psi}_{0}
$$

in these new variables. As $t$ increases indefinitely, each representative point moves in a spiral up the cylinder (i. e. $\lim \bar{\psi}=+\infty$ ).

A point $(\bar{a}, \bar{\theta}, \mathrm{o})$ of the base $\bar{\psi}=\mathrm{o}$ of the cylinder will thus be taken into a definite point $\left(\bar{a}^{\prime}, \bar{\theta}^{\prime}, 2 \pi\right)$ of $\bar{\psi}=2 \pi$ in such a way that

$$
\bar{a}^{\prime}=a, \quad \bar{\theta}^{\prime}=-2 \pi\left[\left(\frac{\bar{a} a_{2}+\mathrm{I}}{a_{2}}\right)^{\frac{3}{2}}+\mathrm{I}\right]^{-1}+\bar{\theta} .
$$

In the case $\mu=0$ the restricted problem of three bodies may be associated with the one-to-one continuous transformation of a circular discoid into itself.

It may be noted that, if we refer the states of motion to the space of inversion in which the representation is two-to-one, the circular disc is replaced by a discoid bounded by the closed curve which represents the retrograde circular orbit.

Now it is clearly possible to establish a one-to-one continuous correspondence between the states of motion in the case $\mu \neq 0$ when a closed oval of zero velocity surrounds $J$, and the states of motion in the case $\mu_{0}=0$, in such wise that the retrograde orbit for $\mu \neq \neq$ of the type established to exist in the preceding paragraph corresponds to the retrograde circular orbit for $\mu=0$. For example, if one continuously deforms the region of possible motion for $\mu \neq 0$, leaving the vicinity of the singular point $J$ unaltered, so that the oval of zero velocity becomes the circle of zero velocity for $\mu=0$, and so that the retrograde orbit for $\mu \neq 0$ becomes the retrograde circular orbit for $\mu=0$, any state of motion for $\mu \neq 0$ may be set in continuous correspondence with the state of motion for $\mu=0$ with which it coincides in position and direction after deformation.

That is, we are entitled to use the representation of the states of motion in the cylinder $\bar{a} \leqslant\left(a_{2}-a_{1}\right) / a_{1} a_{2}$, o $\leqslant \bar{\psi} \leqslant 2 \pi$, for $\mu \neq 0$ also, where the surface of the cylinder is to correspond to the known retrograde orbit, and the individual states of motion along this orbit correspond to the lines $2 \vec{\theta}+\vec{\psi}=$ constant. 
Incidentally let us apply this representation to define the term direct periodic orbit of simple type. It is manifestly desirable to include all those orbits which make a single direct circuit of $J$ in the $x, y$-plane, and also orbits obtained by continuous deformation of such a direct orbit. But in the case $\mu=0$ the axis $\bar{a}=0$ corresponds to the direct circular orbit. A direct periodic orbit (with respect to the given retrograde periodic orbit) of simple type may thus be defined as one which corresponds to a stream line joining the point $(\bar{a}, \bar{\theta}, \bar{\psi})$ with the point $(\bar{a}, \bar{\theta}, \bar{\psi}+2 \pi)$. In general a periodic orbit is represented by a line joining $(\bar{a}, \bar{\theta}, \bar{\psi})$ with $(\bar{a}, \bar{\theta}, \bar{\psi}+2 k \pi)$. Under these circumstances the periodic orbit will be said to make $k$ circuits of the given retrograde orbit. A direct periodic orbit of simple type is accordingly one which makes a single positive circuit of the retrograde orbit $(k=\mathrm{r})$.

For $\mu=0$ every point of the cylinder moves from one end to the other as time increases $(d \bar{\psi} / d t>0)$. This property will certainly continue to hold for $\mu$. sufficiently small. We see that, as long as this is the case, it is possible to construct a discoid across the cylinder which is cut by every stream line in the same sense. To prove this we may use the method used in $\$ I 5 for establishing the corresponding theorem for the transformation of a ring into itself.

The transformation $\bar{T}$ which takes a point $K$ of one of these discoids into a point of the congruent discoid displaced upward by a distance $2 \pi$ constitutes clearly a oneto-one continuous transformation of a discoid into itself of the same general character as the transformation $T$ of a ring-shaped region into itself, and effecting an entirely analogous reduction of the restricted problem of three bodies.

It is not difficult to see that other transformations of the variable $a, \theta, \psi$ can be used to lead us to an infinite number of transformations like $\bar{T}$. The transformation $\bar{T}$ is the only one, however, whose construction depends on a retrograde periodic orbit alone.

If it be recalled that a transformation of a discoid into itself always possesses an invariant point ${ }^{18}$ ) it is clear that $\bar{T}$ possesses an invariant point. To such a point corresponds necessarily a direct periodic orbit of simple type. Now the transformation $\bar{T}$ will exist if and only if every orbit in a sufficient interval of time makes an arbitrary number of positive circuits of the retrograde periodic orbit (cf. $\$$ Is).

If the total number of positive circuits of the retrograde periodic orbit by an arbitrary orbit increases indefinitely with the time t there necessarily exists a transformation $\bar{T}$, and at least one direct periodic orbit of simple type.

As $\mu$ increases from zero, the direct orbit which is the continuation of the direct circular orbit may become unstable by uniting with another direct orbit. This means merely that two invariant points of $\bar{T}$ coincide, but presents no other special feature

I8) L. E. J. BROUWER, Über eineindeutige, stetige Transformationen von Flächen in sich [Mathematische Annalen, Vol. LXIX (I910), pp. I76-18o]. 
of interest so far as $\bar{T}$ is concerned. The transformation $T$ of Polnciate, on the con. trary, depends essentially upon the stability of both the direct and retrograde orbits, and will no longer exist after the direct orbit becomes unstable.

This state of affairs seems to me to make it probable that the restricted problem of three bodies admit of reduction to the transformation of a discoid into itself as long as there is a closed oval of zero velocity about $J$, and that in consequence there always exists at least one direct periodic orbit of simple type.

\section{\20.}

\section{The Symmetric Periodic Orbits.}

We shall now restrict ourselves to the case when $u$. is so small that the transformation $T$ exists and may be represented in the explicit form given in $\ \mathrm{r}_{3}$, although, in reality, it is only the existence of a transformation $T$ in which the boundaries of the ring correspond to two orbits symmetric with respect to the $x$-axis that is essential for our purposes.

We propose to make application of the concept of the ring to prove the existence of infinitely many symmetric periodic orbits, and at the same time to develop briefly their mutual relation.

In order to treat the periodic orbits symmetric with respect to the $x$-axis, we observe first that such an orbit crosses the $x$-axis twice and only twice at right angles. For if the orbit crosses at $t=t_{\mathrm{o}}$ and $t=t_{\mathrm{r}}$ successively at right angles the complete orbit is obtained by a reflection in the $x$-axis and has only two crossings at right angles. Hence there are at most two such crossings.

Moreover if at $t=t_{\mathrm{o}}$ and $t=t_{\mathrm{r}}$ we have symmetric crossings at one and the same point, it is clear that at $t^{\prime}=\left(t_{0}+t_{1}\right) / 2$ we have one crossing at right angles; also if there is one crossing at right angles at $t=t^{\prime}$ which is made again at $t=t^{\prime \prime}$ for the first time, there will be a similar crossing at $\left(t^{\prime}+t^{\prime \prime}\right) / 2$. Hence there are precisely two crossings at right angles.

Now the crossings at right angles may be divided into four classes. The first class corresponds to the states of motion $x>\mu, y=0$ of the ring; guiding ourselves by the analogy with the case $\mu=0$ we shall term these states of motion lower passage at opposition; for $P$ is in opposition to $S$. The second class corresponds to crossing at right angles for $x>\mu, y=0$ not along the ring; this state of motion may be termed higher passage at opposition. The third and fourth classes $(x<\mu, y=0)$ may likewise be termed lower and higher passage at conjunction according as the state of motion belongs to the ring or not.

We shall represent the transformation $T$ on an ordinary ring. This ring may be conceived of as obtained from the ring of $\ 1_{3}$ by a continuous distortion such that the points in the $x$-axis are carried into points of a new axis and such that pairs of 
points symmetrical with respect to that axis are carried over into pairs symmetrical with respect to the new axis. The transformation $T$ may still be written $R U$ where $R$ is a reflection in the axis and $U$ is another transformation of period 2 having essentially the same character as $R$.

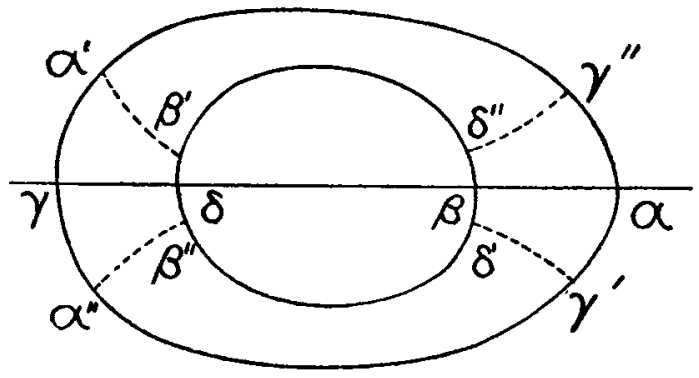

(Fig. 6).

The lower crossings at right angles will be represented by segments $\alpha \beta$ or $\gamma \delta$ of the axis according as the crossing is of the first or third class (fig. 6). Here we shall choose $x, \gamma$ on the boundary corresponding to the direct orbit, and $\beta, \delta$ on the boundary corresponding to the retrograde orbit.

Higher crossings of the second and fourth classes are not represented in the ring. However we may associate with each such crossing the first later state of motion along the same orbit which lies on the ring; these form two continuous lines $x^{\prime} \beta^{\prime}$, $\gamma^{\prime} \delta^{\prime}$ (fig. 7). Two lines $x^{\prime \prime} \beta^{\prime \prime}, \gamma^{\prime \prime} \delta^{\prime \prime}$, symmetrically placed with respect to $x^{\prime} \beta^{\prime}, \gamma^{\prime} \delta^{\prime}$, correspond to the first earlier state of motion along these orbits represented by a point of the ring (fig. 7).

In the case $\mu_{0}=0$ we are in a position to completely determine the position of the curves $\alpha^{\prime} \xi^{\prime}, \gamma^{\prime} \delta^{\prime}, x^{\prime \prime} \xi^{\prime \prime}, \gamma^{\prime \prime} \delta^{\prime \prime}$. Let us employ the variables $a, \theta, \psi$ of $\oint \mathrm{I}$. We first note that higher passage at opposition corresponds to $\theta=\pi, \psi=\pi$. Also equations (37) show us that the motion of a particle for which $\theta=\pi, \psi=\pi$ at $t=0$ is given by

$$
a^{\prime}=a, \quad \theta^{\prime}=-t+\pi, \quad \psi^{\prime}=a^{-\frac{3}{2}} t+\pi
$$

The first point of the ring for $t>0$ is attained for $\psi^{\prime}=2 \pi$, and the curve $\alpha^{\prime} \beta^{\prime}$ has the equation

$$
\theta=\pi\left(\mathrm{I}-a^{\frac{3}{2}}\right)
$$

This curve lies between $\alpha \beta(\theta=0)$ and $\gamma \delta(\theta=\pi)$; for large values of the constant $C$ of JACOBI $a$ is small and the segment $\alpha^{\prime} \beta^{\prime}$ nearly coincides with $\gamma \delta$. Likewise the equation of $\gamma^{\prime} \delta^{\prime}$ is found to be

$$
\theta=-\pi a^{\frac{3}{2}}
$$

Thus $\gamma^{\prime} \delta^{\prime}$ lies between $\gamma \delta$ and $\alpha \beta$; for large values of $C, \gamma^{\prime} \delta^{\prime}$ nearly coincides with $\alpha \beta$. The curves $x^{\prime} \beta^{\prime}$ and $\gamma^{\prime} \delta^{\prime}$ vary only slightly from these positions when $\mu$ is small. 
The points of the curves $\alpha^{\prime \prime} \beta^{\prime \prime}$ and $\gamma^{\prime \prime} \delta^{\prime \prime}$ are invariant under $U$. In fact a point of $\alpha^{\prime \prime} \beta^{\prime \prime}$ or $\gamma^{\prime \prime} \delta^{\prime \prime}$ is carried over into the symmetrical point of $\alpha^{\prime} \beta^{\prime}$ or $\gamma^{\prime} \delta^{\prime}$ by the transformation $T$; for such points are successive points of positive tangency to the auxiliary curves. If, however, we write $T=R U$ we see that the reflection $R$ alone effects this transformation so that $U$ leaves each point of $\alpha^{\prime \prime} \beta^{\prime \prime}$ and $\gamma^{\prime \prime} \delta$ " invariant.

By the aid of this fact we may conceive of $U$ as a distorted reflection of the ring into itself, the axis of the reflection being composed of the segments $\alpha^{\prime \prime} \beta^{\prime \prime}$ and $\gamma^{\prime \prime} \delta^{\prime \prime}$.

What is the condition for a symmetric periodic orbit? Such an orbit is completely characterized by the fact that it has two states of motion, and only two, represented by points on the segments $\alpha \beta, \gamma \delta, \alpha^{\prime} \beta^{\prime}, \gamma^{\prime} \delta^{\prime}$, corresponding to the two crossings at right angles. Hence if a point $K$ of one of these segments is taken into a point $L$ of such a segment by $k$-fold repetition of $T$, the corresponding orbit is symmetric and periodic.

It is seen at once that there exist an infinite number of symmetric periodic orbits with right angle crossings of assigned classes, at least if $\mu$ be small.

In fact let $-\rho$ and $-\sigma$ be the coefficients of rotation along the boundaries of the ring corresponding to the retrograde and direct periodic orbits respectively (see $\$$ I 3 ). Since for $\mu=0$ we have $\rho=2 \pi a_{1}^{\frac{3}{2}}, \sigma=2 \pi a_{2}^{\frac{3}{2}}$ it is clear that $\rho$ and $\sigma$ are unequal $(\rho<\sigma)$ for small $\mu$. After $m$ applications of $T$ the rotation of $\beta, \beta^{\prime}, \delta, \delta^{\prime}$ and $\alpha, \alpha^{\prime}$, $\gamma, \gamma^{\prime}$ will be represented by —mp and — mr respectively. For $m$ large enough, $m \rho$ and $m \sigma$ will differ by an arbitrarily large quantity. The $m$ th images of $x \beta, \gamma \delta, \alpha^{\prime} \beta^{\prime}, \gamma^{\prime} \delta^{\prime}$ will wind around the ring arbitrarily often. To each crossing of these lines themselves by the $m$ th images of any one of the four lines corresponds a symmetric periodic orbit.

I shall now proceed to develop more explicit results concerning the existence and distribution of the symmetric periodic orbits. In order to do so it is first necessary to define two characteristic integers corresponding to each such orbit. It will be assumed in the present paragraph that $\mu$ is so small that the inequalities $o<p<\sigma<2 \pi$ hold. Under this condition no two points of a symmetric pair $\alpha^{\prime}, \alpha^{\prime \prime}$ or $\beta^{\prime}, \beta^{\prime \prime}$ or $\gamma^{\prime}, \gamma^{\prime \prime}$ or $\delta^{\prime}, \delta^{\prime \prime}$ can coincide, for such a coincidence would imply that either $\rho$ or $\sigma$ was a multiple of $2 \pi$; it must be recalled that $\alpha^{\prime}, \beta^{\prime}, \gamma^{\prime}, \delta^{\prime}$ are derived from $\alpha^{\prime \prime}, \beta^{\prime \prime}, \gamma^{\prime \prime}, \delta^{\prime \prime}$ respectively by an application of the transformation $T$. Hence $\alpha^{\prime}, \beta^{\prime}, \gamma^{\prime \prime}, \delta^{\prime \prime}$ continue to lie in the upper half of the ring as at $\mu=0$, and $\alpha^{\prime \prime}, \beta^{\prime \prime}, \gamma^{\prime}, \delta^{\prime}$ continue to lie in its lower half for the same reason (fig. 7).

Suppose that $K$ is a point in which a given symmetric periodic orbit is positively tangent to an auxiliary curve of the ring. Furthermore suppose that $k$ is the least integer such that $T^{k}(K)=K$ so that the orbit is positively tangent to an auxiliary curve of the ring $k$ times before re-entering. Then $k$ will be termed the first characteristic integer of the symmetric periodic orbit; in the case $\mu=0, k$ stands for the number of circuits of the rotating ellipse made, before the orbit closes in the $x, y$-plane.

After $k$ applications of $T$ the point $K$ will have rotated about the ring a certain number of times $l$. The integer $l$ will be termed the second characteristic integer of the 
symmetric periodic orbit; in the case $\mu=0, l$ stands for the number of rotations of the moving $x, y$-plane during a complete circuit of the orbit in that plane.

The quotient $k / l$ measures the mean angular velocity of the particle $P$ about $J$ with reference to fixed axes.

We have now to consider the possible combinations of pairs of the four classes of right angle crossings which may arise, and to ask in the ten possible cases what values of $k$ and $l$ correspond to existing periodic orbits. Inasmuch as the treatment in all cases is essentially the same, the results will only be derived in full for the case in which both crossings are of the first class (lower, at opposition).

Suppose first that $T^{k}(K)=L$ where $K$ and $L$ are distinct points of $\alpha \beta$ corresponding to the two right angle crossings. Then we have

$$
K=T^{-k}(L)=[U R]^{k}(L)=U(R U)^{k-1}[R(L)]
$$

But since $R(K)=K, R(L)=L$, this yields at once $K=T^{k}(L)$. Hence we have $T^{2 k}(K)=K$, as is to be expected. It is also clear that the equation $T^{\lambda}(K)=K$ has $\lambda=2 k$ as its least positive integral solution.

But after $k$ repetitions of $T$ the points $x$ and $\beta$ regress from their initial positions by $k \sigma$ and $k p$ along the inner and outer boundaries of the ring respectively. Consequently, if $2 l \pi$ lies between $k p$ and $k \sigma$, one necessarily infers that the line $\alpha \beta$ in its final position will have some point $K$ which regresses in an angular sense by exactly $2 l \pi$, that is lies at a point $L$ of $\alpha \beta$.

The corresponding periodic orbit will have $2 k$ and $2 l$, or equal submultiples there of, $2 k / \lambda$ and $2 l / \lambda$, for first and second characteristic integers.

For any $k$ and $l$ such that

$$
k_{i}<2 l \pi<k \sigma
$$

there exists a symmetric periodic orbit with $2 k$ and $2 l$ (or equal submultiples thereof) for first and second characteristic integers respectively, and with both right angles crossings of the same class.

The argument given above is made only in the case in which both crossings are lower and at opposition, but applies to the four similar cases without any modification.

Suppose next that $T^{k}(K)=L$ where $K$ lies on $\alpha \beta$ and $L$ on $\gamma \delta$. Here we find at once $T^{2 k}(K)=K$ as before. But after $k$ repetitions of $T, \alpha$ and $\beta$ regress by $k \sigma$ and $k p$ along the boundaries of the ring, and so there will be a point of $\alpha \beta$ which regresses by precisely $(2 l+\mathrm{I}) \pi$ if $(2 l+\mathrm{I}) \pi$ lies between $k p$ and $k \sigma$. Such a point appears as a point $L$ of $\gamma \delta$.

Strictly speaking, the existence of a point which regresses by $(2 l+1) \pi$ in an angular sense has not been proved. If, however, we compare $T^{-k}(\alpha)$ and $T^{k}(\alpha)$, noting that

$$
T^{-k}(\alpha)=[R U]^{k}(x)=[R U]^{k} R(\alpha)=R[U R]^{k}(x)=R T^{-k}(\alpha)
$$

it appears that if $T^{k}(\alpha)$ has regressed from $\alpha$ by less than $(2 l+1) \pi$ in an angular sense, the $T^{-k}(\alpha)$ has advanced from $\pi$ by the same amount. But then the application of $T^{2 k}$ to $T^{-k}(\alpha)$ regresses the point $T^{-k}(\alpha)$ by less than $2 l+$ I complete cycles. 
Hence by the fundamental property of coefficients of rotation such as $\sigma$ ( $\$$ I3), we have

which is impossible.

$$
k \sigma<(2 l+1) \pi
$$

For any $k$ and $l$ such that

$$
k \rho<(2 l+\mathrm{I}) \pi<k \sigma
$$

there exists a symmetric periodic orbit with $2 k$ and $2 l+\mathrm{I}$ (or equal submultiples thereof) for first and second characteristic integers respectively and with right angle crossings of the first and third, or second and fourth classes.

The two similar cases are included in this statement.

In the third place let us suppose that $T^{k}(K)=L$ where $K$ is a point of $x \beta$ and $L$ is a point of $\alpha^{\prime} \beta^{\prime}$. Here we have

$$
K=T^{-k}(L)=[U R]^{k}(L)=U T^{k-1}(R L)=U T^{k-2}(L),
$$

since $R(L)=U R(L)=T^{-1}(L)$. Consequently we have $K=R(K)=T^{k-1}(L)$, and thus finally $T^{2 k-1}(K)=K$. That is, $2 k-\mathrm{I}$ is the first characteristic integer.

Sufficient conditions for such a symmetric periodic orbit with second characteristic integer $2 l+2$ are

$$
k \rho<(2 l+\mathrm{I}) \pi, \quad(2 l+2) \pi<k \sigma .
$$

For, if these conditions are satisfied, after $k$ repetitions of $T$ the point $\alpha$ regresses by $k \sigma$ which will be more than $l+\mathrm{I}$ complete rotations (see above). Similarly $\gamma$ regresses by less than $l+\frac{1}{2}$ rotations. The $k$ th image of a point $K$ of $\alpha \rho$ will therefore surely fall at a point $L$ of $\alpha^{\prime} \beta^{\prime}$, so that $K$ has regressed by $(2 l+1) \pi+d,(d<\pi)$. By an application of $T^{-1}$ this point goes to the symmetrical point of $\alpha^{\prime \prime} \beta^{\prime \prime}$; in this position $K$ has regressed by $(2 l+1) \pi-d$. Now because of the symmetry of $T$ and $T^{-1}$ noted above, a further application of $T^{l}$ will advance the point by $(2 l+2) \pi+d$ more. Hence, in all, the angular regress of $K$ after $2 k-1$ applications of $T$ is precisely $(4 l+2) \pi$.

For any $k$ and $l$ such that

$$
k \rho<(2 l+\mathrm{I}) \pi, \quad(2 l+2) \pi<k \sigma
$$

there exists a symmetric periodic orbit with $2 k-\mathrm{I}$ and $2 l+\mathrm{I}$ (or equal submultiples thereof) for first and second characteristic integers and with right angle crossings of the first and second, or third and fourth classes.

In precisely similar manner we may prove the following result.

For any $k$ and $l$ such that

$$
k \rho<2 l \pi, \quad(2 l+\mathrm{I}) \pi<k \sigma
$$

there exists a symmetric periodic orbit with $2 k-\mathrm{I}$ and $2 l$ (or equal submultiples thereof) for first and second characteristic integers, and with right angle crossings of the first and fourth or second and third classes.

The method above employed can be made to yield a variety of results concerning the distribution of the symmetric periodic orbits. We shall confine ourselves here to 
noting a salient fact concerning the order in which these orbits cross the $x$-axis at right angles. For convenience we shall use the four auxiliary curves $\alpha \beta, \gamma \delta, \alpha^{\prime} \beta^{\prime}, \gamma^{\prime} \delta^{\prime}$, corresponding to the four types of crossing, in stating the results.

If $\left(k_{1}, l_{1}\right)$ and $\left(k_{2}, l_{2}\right)$ are pairs of first and second characteristic integers for two symmetric periodic orbits belonging to points $K_{1}$ and $K_{2}$ of one of the four curves $\times \beta$, $\gamma \delta, \alpha^{\prime} \beta^{\prime}, \gamma^{\prime} \delta^{\prime}$ for $\mu=0$, then symmetric periodic orbits with the same first and second characteristic integers (or equal submultiples thereof) continue to exist with $K_{1}$ and $K_{2}$ in the same order unless, as $\mu$. varies from zero to the given value, one of the rotation numbers $0, \sigma$ passes through the value $2 \pi l_{1} / k_{1}, 2 \pi l_{2} / k_{2}$ respectively.

For $\mu=0$ it has been proved that if $a$ (the semi-major axis of the rotating ellipse) be taken as parameter along the lines $\alpha \beta, \gamma \delta, x^{\prime} \beta^{\prime}, \gamma^{\prime} \delta^{\prime}$, then those orbits are periodic which belong to the states of motion for which $a^{\frac{3}{2}}$ is rational, and that then the ratio of the characteristic numbers $k / l$ is precisely $a^{-\frac{3}{2}}(\$ 10)$.

But these periodic orbits can also be regarded as follows: the $k$ th image of a certain point on one of the lines $x^{p}, \gamma \delta, x^{\prime} \beta^{\prime}, \gamma^{\prime} j^{\prime}$ is taken into a certain point of one of these lines after making $l$ circuits of the ring. To this pair of points corresponds the symmetric periodic orbit. For $\mu=0$ the intersection of the image and curve is unique.

Now let $\mu$ vary from zero. As long as the two curves continue to intersect there will be a periodic orbit with the given $k, l$. An intersection will necessarily exist until a point of intersection passes to the boundary of the ring, since the curves cross but once for $\mu=o$.

At $\mu=0$ there will be but a single point $K_{1}$ and $K_{2}$ with the stated characteristic ratios. When $\mu$ varies from zero a point $K_{1}$ cannot vary through $K_{2}$ for then we should have $k_{1} / l_{1}=k_{2} / l_{2}$. Moreover an even number of points $K_{1}, K_{2}$ will be introduced or disappear by coincidence, corresponding to the intersections of the curve $\alpha \beta, \gamma \delta, \alpha^{\prime} \xi^{\prime}$, $\gamma^{\prime} \delta^{\prime}$ and the images above obtained. Hence points in the same relative order will continue to exist under the specified circumstances.

\section{$\int 2 \mathrm{I}$.}

\section{Some Geometrical Consequences.}

In conclusion I wish to take cognizance of some obvious but curious geometrical consequences of the existence of the ring transformation and the fact that it posseses an invariant area integral (see $\$ 14$ ).

All of these are corollaries of the following fact:

If $\mu$ is taken so small that a transformation $T$ exists, and if $\Gamma$ be any closed curve (not a closed stream line) in the representing sphere $(\$ 7)$ there will necessarily exist an infinite number of stream lines which pass through a point of $\mathrm{\Gamma}$ twice. 
The proof is immediate. If we pass along a stream line from a point $K$ of $\Gamma$ to the first point $L$ of intersection with the ring and then allow $K$ to describe $\mathrm{r}$, the point $L$ will describe either an area enclosing curve $\mathrm{r}^{\prime}$, or an arc of a curve multiply taken, or a single point. The last case is excluded by the hypothesis that $r$ is not a closed stream line. In the second case the truth of the statement made is obvious.

There remains only the first case to consider in which the image $\Gamma^{\prime}$ of $\Gamma$ divides the ring in two or more parts. Now consider the successive image of $\Gamma^{\prime}$ in the ring under the transformation $T$. Because of the existence of a positive integral area invariant over the ring we can infer that $T$ may be repeated so often that some $k$ th image of $\Gamma^{\prime}$ overlaps an $l$ th image of $\Gamma^{\prime}(k>l)$. It follows then that the $(k-l)$ th image of $\Gamma^{\prime}$ overlaps $\Gamma^{\prime}$. Such an image cannot lie wholly within or without $\Gamma^{\prime}$ for that would be in contradiction to the existence of a positive integral area invariant. Through each point of intersection of the boundary of $\Gamma^{\prime}$ and such an image will pass a stream line which intersects $\Gamma$ twice.

By this process $\Gamma^{\prime}$ is broken up into smaller regions (or is transformed into itself, when the statement to be proved is obviously true). Applying again the same argument to these smaller regions we are led to the conclusion that there exist an infinite number of stream lines passing through $\mathrm{r}$ twice.

We include (by convention) the case in which a single orbit cuts $\Gamma$ infinitely often.

We have at once the following corollaries:

a) infinitely many of the orbits pass twice through any given point of the $x$, $y$-plane not on the oval of zero velocity,

b) infinitely many of the orbits pass twice through a point of the oval of zero velocity,

c) infinitely many of the orbits pass through $J$ twice.

For in each case the totality of states of motion under consideration will correspond to a closed line in the representing sphere.

GEORGE D. BIRKHOFF. 